Sol-Gel Derived Ceramic Electrolyte Films on Porous Substrates

LBI -32332

Timothy Walter Kueper

DE:92 016990

(Ph.D. Dissertation)

\author{
Department of Materials Science and Mineral Engineering \\ University of California, Berkeley \\ and \\ Center for Advanced Materials \\ Materials Sciences Division \\ Lawrence Berkeley Laboratory \\ Berkeiey, CA 94720
}

May 1992

This work was supported by the Division of Materials Science, Office of Basic Energy Research, United States Department of Energy, under contract No. DE-AC0376SF00098 with the Lawrence Berkeley Laboratory. 


\title{
Sol-Gel Derived Ceramic Electrolyte Films on Porous Substrates
}

\author{
by
}

Timothy Walter Kueper

\begin{abstract}
A process for the depostion of sol-gel derived thin films on porous substrates has been developed; such films should be useful for solid oxide fuel cells and rclated applications, Yttria-stabilized zirconia films have been formed from metal alkoxide starting solutions. Dense films have been deposited on metal substrates and ceramic substrates, both dense and porous, through dip-coating and spin-coating techniques, followed by a heat treatment in air. X-ray diffraction has been used to determine the crystalline phases formed and the extent of reactions with various substrates which may be encountered in gas/gas devices. Surface coatings have been successfully applied to porous substrates through the control of substrate pore size and deposition parameters. Wetting of the substrate pores by the coating solution is discussed, and conditions are defined for which films can be deposited
\end{abstract}


over the pores without filling the interiors of the pores. Shrinkage cracking was encountered in films thicker than a critical value, which depended on the sol-gel process parameters and on the substrate characteristics. Local discontinuities were also observed in films which were thinner than a critical value which depended on the substrate pore size. A theoretical discussion of cracking mechanisms is presented for both types of cracking, and the conditions necessary for successful film formation are defined. The applicability of these films to gas/gas devices is discussed. 


\section{Chapter 1. Introduction}

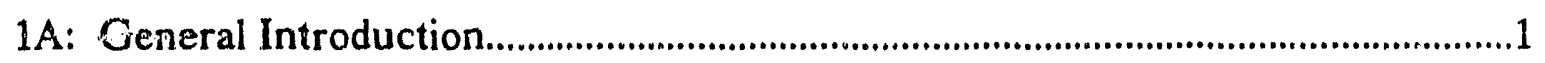

1B: Factors Affecting the Efficiency of Fuel Cells...........................................................2

1C: Other Electrolyte Devices......................................................................................................5

1D: Stabilized Zirconia as a Solid Electrolyte....................................................................

1E: Current Methods of Zirconia Electrolyte Processing.....................................................6

1F: Advantages of the Sol-Gel Process...........................................................................7

1G: Substrates for Consideration in Devices...............................................................9

1H: General Experimental Approach................................................................................12

References......................................................................................................................

\section{Chapter 2: Ceramic Phases Obtained from the Sol-Gel Route}

2A: Metal Alkoxide Starting Solutions...........................................................................20

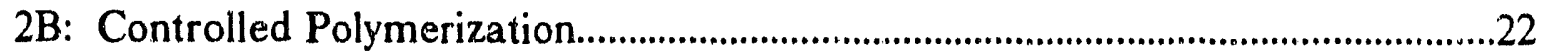

2C: Firing and Crystalline Phase Formation.................................................................24

2D: Substrate Reactions................................................................................................28

2E: Summary of Results....................................................................................................

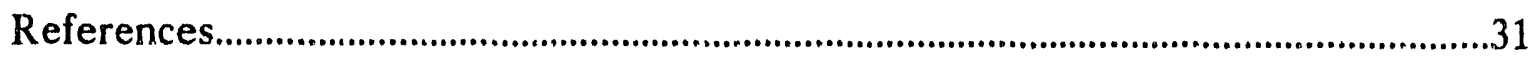

Chapter 3: Effects of Substrate Porosity During Deposition Step

3A: Deposition Techniques.............................................................................................33

3B: Coating Step Experimental Procedure................................................................36

3C: Wetting of Pores During the Deposition Step............................................................37

3D: The Filled-Pore Coating Process..............................................................................40 
3E: Summary of Coating Step Results....................................................................44

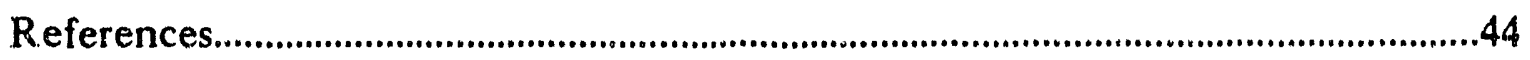

Chapter 4: Mechanical Behavior of Films During Processing

4A: Background: Shrinkage Cracking,.....................................................................46

4B: Description of Shrinkage Cracking on Continuous Substrates................................48

4C: Effect of Substrate Porosity on Cracking Behavior....................................................50

4D. Theoretical Discussion of Cracking Mechanisms....................................................53

4E: Summary of Mechanical Behavior Results............................................................63

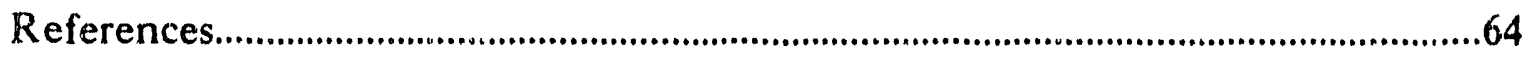

Chapter 5: Suitability of the Process: Electrolyte Device Considerations

5A: Quality of the Electrolyte Film.........................................................................66

5B: Electrode Formation: Alumina Membrane Substrates...........................................68

5C: Use of Graded Porosity Substrates.............................................................................70

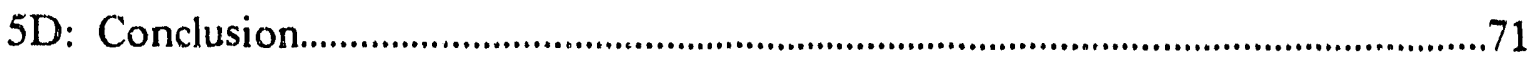

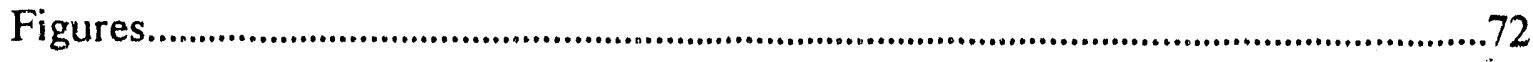




\section{Acknowledgements}

First, I'd like to thank Professor De Jonghe for directing my research; I enjoyed his loose guidance style and intellectual perspective. I'd also like to thank other faculty members who took the time to carefully read this dissertation and make valuable suggestions, Professor Alan Searcy and Professor Angelica Stacy.

I'm also grateful for the advice and efforts of Dr. Steve "Enthusiasm" Visco and Dr. Tom "Encyclopedia of Chemical Technology" Richardson, two scientists without whom I would probably be in graduate school for many more years. I also thank my other group members and coworkers for their help, and for making each and every workday pleasant and productive; at least, that's the way I want to remember it....

Specifically, I want to thank Jeff, Naomi, Shari, and Yves for providing an atmosphere of professional growth, once in a while anyway, but also for their occasional provision of an atmosphere of unprofessional decline, which is essential for proper perspective. In that regard I'd also like to thank some past graduate students; Chi, Dale, Dave, Ding, Mike, and Thea, as well as all Building 62 volleyball players.

The friendships I've made here are much more important and valuable than anything that went into these pages, and I can't attempt to do them justice in this acknowledgement. 


\section{Chapter 1. Introduction}

\section{A: General Introduction}

Fuel cells and gas sensors depend on the production of an external current as a result of differences in the gas composition at the two surfaces of an electrolyte layer. Such devices require a thin layer of ion-conducting material which separates two flowing gases. Among the parameters currently limiting the performance of fuel cells is the ability to obtain an electrolyte layer which is thin enough to have a low ohmic drop but is still continuous so that gas leakage is prevented. The thickness of continuous ceramic layers produced by conventional methods requires these devices to be operated at high temperatures $(1000$ to $1200 \mathrm{C}$ ) to increase the conductivity. In addition, the surfaces of the electrolyte must accommodate electrodes; oxidation or reduction must occur at the gas/electrolyte/electrode triple interface. Practical applications will also require sealing connections around the edges of the electrolyte. As a result of the high operating temperature which is necessary for thick electrolytes, there are severe restrictions on the materials that can be used for electrodes, seals, etc. and/or limitations on device lifetimes.

The sol-gel process is an attractive method for producing very thin, dense ceramic films; continuous films of less than 1 micron thickness have been obtained on flat continuous substrates for a variety of materials $1,2,3$. A fuel cell utilizing such a film could be operated at much lower temperatures (i. e. below $650 \mathrm{C}$ ) with an acceptable ohmic drop. However, for devices which require gas access to both surfaces of the film, a porous film support is needed, adding to the difficulty in solgel film deposition, and complicating the mechanical behavior of the resulting film. In general, the use of a porous substrate will place restrictions on some of the process parameters. Additionally, the characteristics of the substrate porosity also have to be controlled. 
The goal of this research is to study the formation of thin ceramic electrolytes on porous substrates using the sol-gel technique. The phase formation, effect of substrate conditions, and mechanical behavior of the thin films will be discussed. The sol-gel process parameters and substrate pore characteristics which are necessary for successful film formation are defined. The suitability of this process for various electrolyte device applications is also discussed.

\section{B: Factors Affecting the Efficiency of Fuel Cells}

A fuel cell is a device for energy conversion which harnesses the chemical energy supplied by two gases, a fuel gas and an oxidizer. By keeping the gases separate and allowing a controlled reaction to occur through ionic migration in one direction, electrons are forced through an external circuit, providing electric current. This operation depends on the existence of a thin, dense membrane which has good ionic conductivity, negligible electronic conductivity, and is impervious to gas molecules.

One of the most important reasons for the interest in fuel cell technology is the higher theoretical efficiency of a fuel cell over conventional methods of energy conversion which involve Carnot inefficiencies. In practice, this higher theoretical efficiency is always reduced during actual operation. The configuration of the electrolyte layer, electrodes, and pore structure of the support plays a key role in determining the operating efficiency of a fuel cell or related device.

Electrical power is normally generated from heat which is produced by burning fuel. Useful work obtained in this way is limited by the theoretical efficiency of a Carnot cycle; the efficiency depends on the temperature difference which can be produced and is practically limited in most cases to around $45 \%$. (As the temperature is increased, gains begin to be offset by increased frictional losses and heat wastage.) The operation of a fuel cell represents a more direct conversion 
of energy, as the Carnot cycle is avoided. In a hydrogen fuel cell, hydrogen is consumed according to the reaction:

$$
\mathrm{H}_{2}+1 / 2 \mathrm{O}_{2} \rightarrow \mathrm{H}_{2} \mathrm{O}
$$

The theoretical efficiency, the ratio of the work obtained to the energy consumed, depends on the thermodynamics of the reaction. The work obtained can be computed from the Nernst equation, which dictates the voltage set up by the reaction:

$$
E=\frac{\Delta G}{n F}=\frac{R T}{n F} \ln \frac{P^{i}}{P^{\prime \prime}}
$$

where $R$ is the gas constant, $T$ is the temperature, $\mathrm{n}$ is the number of electrons transferred, $F$ is the Faraday constant, and $\mathrm{P}^{\prime}$ and $\mathrm{P}^{n}$ are the partial pressures of oxygen at the air and fuel electrodes, respectively. For exothermic reactions, the efficiency ${ }^{4}$ can be described by:

$$
\eta=\frac{-\Delta G}{-\Delta H}=1 \frac{T \Delta S}{\Delta H}
$$

Inefficiency, in the form of heat wastage, comes only from the entropy term in the free energy, which of course includes a temperature factor. Thus, fuel cells which can be operated at lower temperatures will have a higher theoretical efficiency:

The use of a thin electrolyte layer, then, by allowing a fuel cell to operate at a lower temperature, will increase the thermodynamic efficiency of the cell. The cell also can be used more effeciently because less insulation is needed and thermal cycling time is reduced. In addition, the lifetime and stability of the device are enhanced by a lower operating temperature: Thermal stresses are reduced, degradation reactions are slowed, and electrode sintering can be reduced. Lowertemperature cells also have economic advantages; construction may be cheaper because metals might be used in place of ceramics for many of the components, and more options would be available for the choice of sealing materials. 
There are some disadvantages to a lower operating temperature. The rates of electrode reactions and gas diffusion will decrease with decreasing temperature, which will reduce the current density at which the device is capable of operating without dramatic voltage losses due to polarization. The electrode reactions become the chief concern as the temperature is lowered 5,6 , and improvement of reaction rates generally requires the use of more expensive catalytic electrodes. However, new materials are being researched which may improve the options available $5,6,7$. Another disadvantage of a lower operating temperature is the restriction it places on the fuel which can be used: While free hydrogen is required at the fuel electrode, in high temperature cells $(1000 \mathrm{C})$ hydrogen can be obtained directly as an in-situ reaction product of a more convenient fuel such as methanol, ammonia, or hydrocarbon fuels. Hydrogen-producing reactions are unfavorable at lower temperatures for methane, so that a low temperature cell would probably require a conversion facility to supply hydrogen.

The structure of the porosity in the support and electrode is also important for the successful operation of a fuel cell. Electrode reactions must take place at the triple interface between the electrolyte, electrode, and gas phase. The total length of this triple interface should be as great as possible in order to allow a high current density. To this end, the pore structure should be very fine where it meets the surface of the electrolyte. To allow adequate gas supply to the interface, however, the pores should have large diameters, so that the cross-section is large in comparison with the mean free path of the gas molecules, and Knudson diffusion conditions are avoided 8 . Thus, an ideal support material would have a graded pore structure, with large pores leading to finer pores at the electrolyte surface. 


\section{C: Other Electrolyte Devices}

Electrolyte membranes with similar qualities to those desired for fuel cells are needed in a variety of devices, including gas sensing applications and gas separation devices. Gas sensors operate like fuel cells in that a difference in gas composition leads to a voltage between the two surfaces of the electrolyte. Gas separators, on the other hand, have the reverse function of creating concentration changes in the two separated gasses through the use of an applied voltage. Both applications are governed by the same equation which describes the voltage of a fuel cell as described above. While most applications involve the use of oxygen ion conductors, for some applications involving hydrogen reactions, proton conductors such as doped strontium cerium oxide ${ }^{9}$ are desirable.

While pure ionic conductors are the primary type of solid electrolyte used in devices, applications also exist for mixed (ion/electron) conductors. An oxygen separation membrane could be made from a simple mixed conductor without electrodes; if oxygen on one side of the electrolyte has a higher activity (as, for example, in pressurized air), it could be reduced at the surface of the membrane and the ions could diffuse to the other surface accompanied by the electrons, where molecular oxygen would again form. Relative to electrode-containing devices, such a device would have simplified processing and relaxed requirements for compatibility, but would still need to be supported by a porous substrate. Deposition of continuous sol-gel derived films on porous substrates, as carried out in this research, may represent a clearing of the last major obstacle for this particular technology.

\section{D: Stabilized Zirconia as a Solid Electrolyte}

The characteristics of the ideal material for thin-film oxygen ion-conducting solid electrolyte applications include low resistance and pure ionic conduction at 
high temperatures, so that the device will have high efficiency as discussed above. In addition, electrolytes must be chemically inert at these temperatures with respect to a variety of materials which may be used as electrodes, supports, and seals or gas manifolds. Mechanical strength and thermal shock resistance are also important for the reliability of the device. Among known oxygen ion conductors, yttria-stabilized zirconia has the best combination of these properties 10,5 , and has been chosen as the base material for this research.

Zirconia achieves optimum conductivity at a yttria doping level that corresponds to full cubic stabiliztion; this composition is approximately 9 mole percent $\mathrm{Y}_{2} \mathrm{O}_{3}$, at which the conductivity activation energy is between 0.8 and 0.9 $\mathrm{eV} 11$ 12. The conductivity at $1000 \mathrm{C}$ is 0.10 to $0.12 \mathrm{ohm}^{-1} \mathrm{~cm}^{-1}$. Electrical conductivity is independent of oxygen partial pressure over a wide temperature range; conduction is truly electrolytic, with the electron transport number much less than $1 \%$.

\section{E: Current Methods of Zirconia Electrolyte Processing}

There has been a large research effort to find a method of producing zirconia films which improves upon normal powder processing methods. Zirconia coatings for electrolyte applications have been deposited on porous substrates using a variety of novel methods, including electrochemical vapor deposition (EVD) ${ }^{13}, \mathrm{RF}$ sputtering 14 , and spray pyrolysis 15 . While these approaches can overcome some of the disadvantages associated with powder techniques, the morphological control remains unsatisfactory; EVD and spray pyrolysis required minimum film thicknesses of roughly 30 microns before gas leakage was prevented, while RF sputtering led to a 5 micron film which had substantial gas leakage and in some cases did not prevent electronic shorts through the film when electrodes were 
attached. No coating has yet been reported which will allow efficient cell operation at low $(650 \mathrm{C})$ temperatures.

\section{F: Advantages of the Sol-Gel Process}

Most conventional processing techniques are unsuitable for producing thin, dense, and continuous solid electrolyte films; the sol"gel route offers many advantages.

\section{Description of the Sol-Gel Route}

A brief description is given here; the individual steps will be considered in more detail in later chapters.

Alkoxides of the desired metals are generally used as the starting materials in the sol-gel process, and are dissolved in an organic solvent. The conversion of the solution to a gel begins with the addition of water, leading to a hydrolysis reaction. For example,

$$
\mathrm{Zr}\left(\mathrm{OC}_{3} \mathrm{H}_{7}\right)_{4}+\mathrm{H}_{2} \mathrm{O} \rightarrow \mathrm{ZrOH}\left(\mathrm{OC}_{3} \mathrm{H}_{7}\right)_{3}+\mathrm{HOC}_{3} \mathrm{H}_{7}
$$

Hydrolyzed molecules can further undergo a condensation reaction, in which an oxygen link between two metals is formed. Hydrolysis and condensation reactions continue to build larger polymers, which at some point link together in a continuous network, creating a gel.

At some stage during the gelation process, the solution has a suitable viscosity for film deposition. Two techniques, dip coating and spin coating, are widely used. Dip coating, in which the substrate is submerged in and then slowly withdrawn from the solution, is effective for the uniform coating of large surface areas. The thickness of the coating can be controlled through manipulating the viscosity and the speed and angle at which the substrate is withdrawn. The spin coating process consists of placing a small pool of liquid on the substrate and then 
spinning it at high speed to distribute the liquid in a thin layer. The spinning speed and the viscosity of the solution control the thickness of the coating 16 .

Either deposition method will leave a thin layer of gel on the substrate. The gel at this stage consists of polymer and some amount of solvent; the evaporation of the solvent can be very rapid and can be almost completed during the deposition step. Any remaining solvent is removed during the heat treatment.

After solvent removal, continued heating causes the burnout of residual organic groups, which can be accomplished by a low temperature hold or by slow heating to the crystallization temperature range. There should be some concern about residual carbon in the electrolyte, which may reduce the efficiency of electrolyte devices. Crystallization occurs at low temperatures (400-600 C), giving a dense structure without need for high temperature sintering 17.

\section{Advantages Over Other Processes}

When compared with techniques relying on the consolidation of powders (tape sasting, for example), sol-gel films tend to allow increased purity and homogeneity, much lower sintering temperatures, and increased control of thin film morphology. Starting materials for sol-gel processing are usually chemicals in solution, which can be made purer than bulk powders by utilizing such processes as vacuum distillation or crystallization. Additionally, powder processing can introduce impurities, e. g. as a consequence of grinding operations or as sintering additives needed to control grain growth. In the sol-gel route, grinding processes are absent and grain growth is strongly supressed at the reduced sintering temperature, so the product is allowed to retain its high purity. Homogeneity is also easier to achieve for the sol-gel route, as the components can be mixed on a molecular scale in solution rather than through the mixing of powders. The reduced sintering temperature and increased morphology control are a direct result of the nature of 
the gel formation. Where powder processes are limited in their ability to pack particles in a thin dense film, in the sol-gel process the film forms directly from a homogeneous liquid so that there is no inherent packing porosity or minimum thickness based on particle size.

The main disadvantages of the sol-gel process are the expense of the chemicals used and the large shrinkage inherent to the process. Together, these characteristics have made preparation of bulk objects impractical. However, the expense of the chemicals is not important when small volumes are used to make thin films. In addition, while shrinkage cracking is a definite problem, it appears that within certain guidelines crack-free films can be obtained (see Chapter 4).

\section{0: Substrates for Consideration in Devices}

\section{Lanthanum Strontium Manganese Oxide}

Possible substrates for solid electolyte devices must fulfill several tasks, which inlude providing mechanical support, allowing gas to flow freely, providing a means of electronic current collection from the surface of the electrolyte, and serving as a site for catalysis of surface reactions (the nature of which depend on the operating temperature). One approach is to look for materials which can perform all of these functions at once:

Doped Lanthanum strontium manganese oxide (LSM) can be prepared as a thin porous layer with highly open porosity, fair mechanical strength, and good electronic conductivity. Catalytic behavior, although still being investigated, is encouraging 18,19 . Some samples of tape-cast LSM were used as substrates in this research, and their suitablility is discussed in Chapter 3. 


\section{Controlled-Porosity Alumina Membranes}

An alternate approach to substrate fabrication is to use a material which provides a stable mechanical support and highly aligned porosity by itself, and to coat it in some way with another material which can provide electronic conduction and catalytic properties, thus taking advantage of the most desirable properties of both materials.

Alumina membranes are available with highly controlled porosity which is suitable for high gas flow-rate applications. The membranes have a linear, parallel arrangement of large pores extending in from one face, which through manipulation of the fabrication process can be made to branch into smaller pores, leading to a finer scale porosity at the opposite face. The surface with the fine scale porosity represents a highly controlled substrate which may be necessary for sol-gel film formation (as discussed in Chapter 4). The fineness of the porosity also maximizes the total length of the triple interface, which may be important for determining the current density at which the device can operate. The larger scale porosity in the remainder of the filter allows for increased gas flow rates. The relative pore sizes and thicknesses of the separate regions of the filter can be tailored to suit the desired application. The membranes which have been in use in this research, which are normally for filtration applications, have pores with an initial diameter of 0.2 microns present in a region which has a 52 micron thickness, followed by a layer of 0.02 micron diameter pores which is less than one micron thick. The total porosity is roughly $50 \%$ of the membrane volume.

The membranes are formed by anodizing an aluminum metal substrate to create a porous oxide coating which is then separated from the substrate 20 . The pore diameter and spacing is controlled by the applied voltage. The depth of the pores (and hence the thickness of the membrane) is determined by the amount of time the voltage is applied. By a reduction in the applied voltage, the pores can be 
made to branch, providing a new layer of finer scale porosity which is connected to the larger pores. There is always a thin barrier layer of oxide present between the ends of the advancing pores and the aluminum metal. When the voltage is reduced to a very low value, this barrier is extremely thin, and can be readily dissolved to separate the oxide film from the metal substrate. Depending on the chosen thickness of the film, it can either be removed from the electrolyte as a free membrane or else must be supported by a larger-scale porous substrate. The membranes, when provided as small $(25-50 \mathrm{~mm}$ diameter) filters, are 50 to 60 microns thick and have sufficient strength to be handled while unsupported. Larger sheets are supplied with a polymer support due to their fragility. Thinner membranes (1-5 microns), regardless of size, would need to be supported during handling and use.

The thermal and chemical stability of alumina make these membranes attractive for intermediate-temperature fuel cell applications. Alumina retains its rigidity and is stable at high temperatures, and has a similar thermal expansion coefficient to zirconia. The membranes appear to have pore walls with a dense micro-crystalline structure which should have good dimensional stability and no sintering or grain growth at intermediate temperatures $(650 \mathrm{C})$. The chemical stability of alumina also allows these membranes to remain unaffected by exposure to solvents during device processing steps such as sol-gel deposition and electroless plating.

The availability of large-area membranes is limited only by handling difficulties due to their fragility; the anodization process can be applied to any size of aluminum metal substrate. Membranes are currently commercially available in $4.5 \times 7.5$ inch sheets which are used in cross-flow filtration applications. Large sheets are sectioned or cut into desired shapes by a laser etching process. 
These alumina membranes have been used as the substrate material in the bulk of this research. Possible processes for the addition of electronically conductive coatings are discussed in Chapter 5.

\section{Other Substrates}

A similar approach to the graded-porosity anodization product described above is the use of size-graded powders in the fabrication of a controlled-porosity substrate. The main disadvantage to the use of alumina membranes is their lack of electronic conductivity, which complicates cell design (see Chapter 5); a gradedporosity substrate composed of conductive material would greatly simplify device fabracation. Possible material choices for such a substrate include conductive ceramics such as LSM, porous metals, or cermets such as nickel-zirconia. Properties such as electronic conductivity, thermal expansion, and catalytic behavior could be optimized with the proper material choice. Control of the porosity to allow continuous sol-gel film formation probably represents the largest challenge for this type of substrate.

\section{H: General Experimental Approach}

The sol-gel process has been used to create the films in this research. Details of the experimental steps will be presented in later chapters. In this section, a brief general description will be given, with emphasis on the more challenging aspects of the process.

\section{Sol-Gel Processing}

Several oxide systems were studied in this research. Initially, the titania system was used to check equipment and procedures for the general sol-gel technique, as this system has been extensively studied and is well-documented 21,22 
23. After this check was accomplished, systems which were of interest for solid electrolyte applications were studied. The bulk of the experimental work has been performed on zirconia electrolyte systems. Basic methods for sol-gel preparation of stabilized zirconia, which can be found in the literature $24,25,26$, have been modified as needed for depositon on porous substrates while maintaining the desired electrolyte characteristics. In addition, some work was done on the formation of the complex oxide, $\mathrm{SrCeO}_{3}$, which is of interest as a proton conductor. Details are presented in Chapter 2.

\section{Gelation}

An attempt was made to control gelation conditions in order to minimize film shrinkage and later cracking. The extent of hydrolysis and condensation reactions can be controlled by the amount of water added and also by the addition of chelating ligands, which can block hydrolysis sites. Three-dimensional polymerization (leading to a rigid gel) can be avoided by leaving an average of only two hydrolysis sites per metal atom unblocked; in this way a linear polymeric structure is obtained, which has important consequences for the flow properties of the gel27. In addition to reducing the viscosity of the gel, the selective blocking of hydrolysis sites can also enable the gel to collapse to a thin film as the solvent evaporates rather than to retain its three-dimensional rigidity. In this way, most of the shrinkage will take place normal to the plane of the substrate, leaving the film in a relatively unstressed condition. Rapid solvent evaporation also improves the collapsibility of the film by reducing the time during which the gel network can age, or create crosslinks that increase the rigidity. The network should also be as dense as possible after solvent evaporation, so that shrinkage during burnout is reduced--thus the bulkiness of side groups and blocking ligands should be kept to a minimum. 


\section{Solution Flow in Porous Substrates}

Most coating solutions will tend to wet the ceramic substrates, such as alumina, which are likely to be used as porous supports in solid electrolyte devices, and capillary pressure will favor flow of the solution into the pores. This problem could possibly be handled by several indirect methods, including surface energy manipulation, blocking of pores with a volatile filler material before coating, or the use of particulate sols.

The approach chosen in this research was to allow the coating solution to begin flowing into the pores. It will be shown in Chapter 3 that while initial saturation due to capillary pressure is very rapid for even extremely small pores, subsequent leakage of the coating solution through the substrate is extremely slow for pores under 10 microns. Thus for thin substrates with small pores, an insignificant volume of coating solution would be drawn into the pores, and film formation should be unaffected. Due to the dilution of the coating solution (the effective zirconia content is typically less than $1 \mathrm{M}$ ), the final volume of zirconia formed is less than $1 \%$ of the solution volume. Small pores that have initially been filled with solution will thus be relatively free of solid zirconia, and the gas/electrolyte/substrate triple interface should remain effectivly at the surface of the substrate.

Fracture cross sections of different coated substrates have been examined to determine the extent of zirconia formation within the pores (see Chapter 3 ).

\section{Control of Cracking and Film Continuity}

During the transformation from a coated solution to the final crystalline product, a significant shrinkage occurs which is resisted by the constraint of the substrate. While much of the shrinkage will take place during solvent evaporation when the material is still able to flow to relieve stresses, significant strain will also 
accompany burnout of organics and crystallization. The increasing film rigidity will then lead to residual stresses. In this research, conditions have been controlled to determine when these stresses can be minimized and when shrinkage cracking can be avoided.

By far the most important parameter for the elimination of cracking is the thickness of the film, as described in the literature $28,29,30$. In this study cracking behavior has been examined as a function of the film thickness, which can be controlled by the altering the viscosity of the coating solution or changing the deposition conditions. Adhesion to the substrate plays an important role in the energy balance that describes unstable cracking, and the effect of varying the substrate characteristics has been observed.

Film continuity also needs to be addressed. For flat, dense substrates lack of continuity should only be a problem for extremely thin films which may be unstable to breakup via diffusional mechanisms 31 . For porous substrates, however, the coating step may not leave a continuous liquid film above pore openings. The relationship between the film thickness and the size of surface features is critical for determining whether films will be continuous. In this research, substrates with different pore dimensions have been tested in order to analyze this relationship.

A complete discussion of cracking will be presented in Chapter 4 .

\section{Film Characterization}

Crystallization behavior of the film as a function of solution composition and treatment temperature have been examined using $X$-ray diffraction (XRD). The effect of the addition of blocking ligands has also been determined.

Film morphology and cracking behavior have been characterized using Scanning Electron Microscopy (SEM); continuity was also quantified by helium/vacuum leak testing of edge-sealed samples. The effects of varying the 
coating parameters and the substrate porosity were monitored by observing such characteristics as cross-sectional thickness, delamination behavior, and film microstructure.

For electrical characterization, films were deposited on metallic or metalcoated substrates which could act as one electrode. A metal coating deposited over the film could act as a second electrode. Ongoing work on this project includes impedance spectroscopy which will analyze the phenomena which contribute to the resistivity, but that characterization will not be discussed in this dissertation.

\section{References--Chapter 1}

1 A. Makishima, H. Kubo, K. Wada, Y. Kitami, and T. Shimohira, "Yellow Coatings Produced on Glasses and Aluminum by the Sol-Gel Process," J. Am. Ceram. Soc., 69 [6] C-127-C-129 (1986).

2 R. Xu, Y. Xu, C. J. Chen, and J. D. Mackenzie, "Sol-gel processing of Strontiumbarium niobate ferroelectric thin film," J. Mater. Res., 5 [5], 916-918 (1990).

3 W. W. Davison and R. C. Buchanan, " $\mathrm{ZrO}_{2}$ Thin Films for Packaging and Microelectronics Applications," pp. 513-521 in Advances in Ceramics Vol. 26, ed. M. F. Yan, K. Niwa, H. M. O'Bryan, Jr., and W. S. Young, The American Ceramic Soc., Inc., 1989.

4 McDougall, A., Fuel Cells, John Wiley and Sons, New York, 1976.

5 H. S. Issacs, "Zirconia Fuel Cells and Electrolyzers," pp. 406-418 in Advances in Ceramics Vol. 3, ed. A. H. Heuer and L. W. Hobbs, the American Ceramic Soc., Inc., 1981.

6 B. C. Steele, J. Drennan, R. K. Slotwinski,N. Bonanos, and E. P. Butler, "Factors Influencing the Performance of Zirconia-Based Oxygen Monitors", pp. 286-309 in Advances in Ceramics Vol. 3, ed. A. H. Heuer and L. W. Hobbs, the American Ceramic Soc., Inc., 1981. 
7 S. P. Badwal, M. J. Bannister, and W. G. Garrett, "Low-Temperature Behavior of $\mathrm{ZrO}_{2}$ Oxygen Sensors", pp. 598-606 in Advances in Ceramics Vol. 12, ed. N.

Claussen and A. H. Heuer, the American Ceramic Soc., Inc., 1984.

8 G. H. Geiger and D. R. Poirier, Transport Phenomena in Metallurgy, p. 463-472, Addison Wesley, Reading, MA (1973).

${ }^{9}$ H. Iwahara, "High Temperature Proton Conducting Oxides and their Applications to Solid Electrolyte Fuel Cells and Steam Electrolyzer For Hydrogen Production," Solid State Ionics, 28-30 573-578 (1988).

10 E. C. Subbarao, "Zirconia--an overview," pp. 1-24 in Advances in Ceramics Vol. 3, ed. A. H. Heuer and L. W. Hobbs, the American Ceramic Soc., Inc., 1981.

11 J. M. Dixon, L. D. LaGrange, U. Merton, C. Miller, and J. Porter II, "Electrical Conductivity of Stabilized Zirconia at Elevated Temperatures", J. Electrochem. Soc. 110 [4] 276-80 (1963).

12 D. W. Strickler and W. G. Carlson, "Ionic Conductivity of Cubic Solid Solutions in the System CaO- $\mathrm{Y}_{2} \mathrm{O}_{3}-\mathrm{ZrO}_{2}$ ", J. Am. Cer. Soc. 47 [3] 122-27 (1964).

13 A. O. Isenberg, "Energy Conversion Via Solid Oxide Electrolyte Electrochemical Cells at High Temperatures," Solid State Ionics, 3/4 431-437 (1981).

14 A. Negishi, K. Nozaki, and T. Ozawa, "Thin-Film Technology for Solid Electrolyte Fuel Cells by the RF Sputtering Technique," Solid State Ionics, 3/4 443-446 (1981).

15 T. Setoguchi, M. Sawano, K. Eguchi, and H. Arai, "Application of the Stabilized Zirconia Thin Film Prepared by Spray Pyrolysis Method to SOFC," Solid State Ionics, 40/41 502-505 (1990).

16 L. E. Scriven, "Physics and Applications of Dip Coating and Spin Coating," pp. 717-729 in Materials Res. Symp. Proc. Vol. 121, ed. C. J. Brinker, D. E. Clark, and D. R. Ulrich, Mat. Res. Soc., 1988.

17 G. C. Frye, A. J. Ricco, S. J. Martin, and C. J. Brinker, "Characterization of the Surface Area and Porosity of Sol-Gel Films Using SAW Devices," pp. 349-354 in Materials Res. Symp. Proc. Vol. 121, ed. C. J. Brinker, D. E. Clark, and D. R. Ulrich, Mat. Res. Soc., 1988. 
18 Y. Ohno, S. Nagata, and H. Sato, "Effect of Electrode Materials on the Properties of High-Temperature Solid Electrolyte Fuel Cells", Solid State Ionics, 3/4 439-442 (1981).

19 T. Inoue, K. Eguchi, T. Setoguchi, and H. Arai, "Cathode and Anode Materials and the Reaction Kinetics for the Solid Oxide Fuel Cell", Solid State Ionics, 40/41 407-410 (1990).

20 R. C. Furneaux, W. R. Rigby, and A. P. Davidson, U. S. Patent \#4,687,551 Aug. 18, 1987.

21 T. Yoko, K. Kamiya, and S. Sakka, "Photoelectrochemical Properties of $\mathrm{TiO}_{2}$ Films Prepared by the Sol-Gel Method", Yogyo-Kyokai-Shi 95 [2] 150-155, (1987).

22 Y. Takahashi and Y. Matsuoka, "Dip-Coating of $\mathrm{TiO}_{2}$ Films Using a Sol Derived from $\mathrm{Ti}(\mathrm{O}-\mathrm{i} \text {-Pr })_{4}$-diethanolamine- $\mathrm{H}_{2} \mathrm{O}$-i-PrOH System", J. Mat. Sci. 23 2259-2266 (1988).

23 A. Tsuzuki, H. Murakami, K. Kani, S. Kawakami, and Y. Torii, "Preparation of Nb-doped $\mathrm{TiO}_{2}$ Films by the Sol-Gel Method", J. Mat. Sci. Lett., 9 624-626 (1990).

24 B. Fegley, Jr., P. White, and H. K. Bowen, "Processing and Characterization of $\mathrm{ZrO}_{2}$ and $\mathrm{Y}$-Doped $\mathrm{ZrO}_{2}$ Powders," Am. Cer. Soc. Bull., 64 [8] 1115-1120 (1985).

25 T. Yogo, "Synthesis of Polycrystalline Zirconia Fibre with Organozirconium Precursor," J. Mater. Sci., 25 2394-2398 (1990).

26 B. Dubois, D. Ruffier, and P. Odier, "Preparation of Fine, Spherical YttriaStabilized Zirconia by the Spray-Pyrolysis Method," J. Am Ceram. Soc., 72 [4] 713715 (1989).

$27 \mathrm{~J}$. Livage, "Synthesis, Structure, and Applications of $\mathrm{TiO}_{2}$ Gels," pp. 717-724 in Materials Res. Symp. Proc. Vol. 73, ed. C. J. Brinker, D. E. Clark, and D. R. Ulrich, Mat. Res. Soc., 1986.

28 S. Sakka, K. Kamiya, K. Makita, and Y. Yamamoto, "Formation of Sheets and Coating Films From Alkoxide Solutions," J. Non-Cryst. Solids, 63 223-235 (1984).

${ }^{29}$ I. Strawbridge and P. F. James, "The Factors Affecting the Thickness of Sol-Gel Derived Silica Coatings Prepared by Dipping," J. Non-Cryst. Solids, 86 381-393 (1986). 
30 A. Atkinson and R. M. Guppy, "Mechanical Stability of Sol-Gel Films," J. Mat. Sci. 26 3869-3873 (1991).

31 K. Miller, F. Lange, and D. Marshall, "The Instability of Polycrystalline Thin Films: Experiment and Theory", J. Mater. Res. 5 [1] 151-160 (1990). 


\section{Chapter 2: Ceramic Phases Obtained from the Sol-Gel Route}

This section begins with some background on sol-gel chemistry. Results from $\mathrm{X}$-ray diffraction studies of the ceramic phases present in the sol-gel derived films are presented next. Results of substrate reaction experiments are then discussed for a variety of substrates which may be encountered in devices.

\section{A: Metal Alkoxide Starting Solutions}

In the preparation of sol-gel films for this research, metal alkoxides were used almost exclusively as starting materials. Metal alkoxides are widely utilized as precursors to ceramic materials because of their ability, in general, to form fine structures as final products.

Systems of metal alkoxides dissolved in organic solvents are subject to hydrolysis, or the attachment of a hydroxyl ion to a metal atom in the place of an organic group. Hydrolyzed molecules can further undergo condensation reactions, which when combined with further hydrolysis leads to the formation of polymeric chains. With the growth of polymers, a gradual transition to a gel takes place in the mixture; this behavior is desired because it allows the mixture to be cast easily and to form a uniform ceramic material after firing.

Transition metal alkoxides are distinguished from main group metal alkoxides by their greater reactivity and by their ability to undergo coordination expansion. Their greater reactivity is due to the lower electronegativity of transition metals, which causes them to be less stable towards hydrolysis and condensation reactions. As a result, they have a tendency to undergo rapid reactions and to form precipitates rather than homogeneous gels. However, polymeric gels can still be obtained by strictly controlling the moisture content of the mixture or by modifying the alkoxide ligands so that they are less reactive. Unlike main group metals, transition metals are often coordinatively unsaturated, and may be able to expand 
their coordination through nucleophilic association mechanisms. These rearrangements can represent a further contribution to enhanced condensation rates.

\section{Modifying Ligands}

The metals studied in this research include zirconium, yttrium, strontium, and cerium, the oxides of which are attractive for use in solid electrolyte devices. These metals are quite reactive, and in order to control the hydrolysis and condensation of the unaltered alkoxides, the moisture control would have to be so strict that use of unaltered alkoxides becomes impractical. While initial preparation of the coating solutions using a dry atmosphere glove box with carefully dried solvents is readily accomplished, moisture control during deposition and avoidance of substrate moisture would require elaborate procedures. Therefore, the strategy of chemically modifying the alkoxide precursors was adopted. In addition to rendering the alkoxides less reactive, in some cases modification also has the advantage of giving the alkoxides a higher solubility.

Diethanolamine (DEA) and triethanolamine (TEA) have been shown to be effective for stabilizing transition metal alkoxides in solution 1 . Ethanolamines have bonding sites with a strong dative nature, and effectively retard the precipitation of oxides and hydroxides in alcoholic solution when excess water has been added ${ }^{1}$. It has also been proposed 2 that stabilization may occur due to ligand extension: The addition of acetic acid to an alkoxide solution may lead to the following exchange:

$$
\mathrm{M}-\mathrm{OR}+\mathrm{CH}_{5} \mathrm{COOH} \rightarrow \mathrm{M}-\mathrm{O}-\mathrm{COCH}_{3}+\mathrm{ROH}
$$

Such a ligand extension, in addition to preventing localized condensation, can increase the solubility of alkoxides. In this research, the addition of DEA to the isopropanol solutions of zirconium isopropoxide, strontium isopropoxide, and 
cerium isopropoxide was found in each case to increase solubility and reduce sensitivity to moisture.

\section{Refluxing}

The goal of most sol-gel processing, of course, is to create a very finestructured ceramic precursor material which can be converted to the desired crystalline phase at low temperatures. Complications may arise for multicomponent oxides. While mixing of the alkoxides of different metals should initially take place on a molecular scale, it is possible for segregation to occur during the hydrolysis and condensation stages. Samples which were prepared with both strontium and cerium in an attempt to create $\mathrm{SrCeO}_{3}$ were unsuccessful; it was found that the two metal alkoxides had dramatically different hydrolysis rates, leading to separate oxide phases.

One possible technique for obtaining a more intimate mixture of these components is a refluxing treatment. During such a holding period at an elevated temperature, random combination of the two metal types should take place if their electronegativities are similar and if their ionic radii are similar ${ }^{3}$. Two metal alkoxides which are capable of increasing their coordination number may also form double alkoxides if they are allowed to react during refluxing in the presence of the parent alcohol ${ }^{4}$. Ongoing work on the strontium cerium oxide system includes the utilization of such conditions, but will be reported elsewhere ${ }^{5}$. For the yttriumdoped zirconium oxide system, refluxing has been found to be unnecessary; as described below, the two metals appear to be intimately mixed in the final product.

\section{B: Controlled Polymerization}

The solution can begin transforming into a solid through polymerization or precipitation, or simply by solvent evaporation and subsequent pyrolysis. If a fine 
colloidal structure is formed in the solvent, the process is referred to as gelation. For fast rates of hydrolysis and condensation, however, large precipitates can form instead, leading in some cases to free powders instead of dense gels. For the purposes of this work, slow rates of hydrolysis and condensation are used to gain the desired fine-structured and dense final product. The fineness of the product can be assessed by noting the firing temperature necessary to produce a dense crystalline ceramic material.

Finely branched structures can be achieved during polymerization if a number of chemical effects are taken into consideration: First, the choice of ligand type on the alkoxide as well as the solvent chosen can affect the size of the metal complex which is stable in solution. Zirconium isopropoxide in isopropanol is the system which produced the product with the finest structure in this work; in this system the alkoxide is predicted to be dimeric in solution, whereas in other solvents zirconium isopropoxide may be present in larger complexes 6 .

A second means of controlling the extent of polymerization is through limiting the moisture content. All solvents were carefully dried over activated alumina, and solution preparation was done in a helium atmosphere glove box (Vacuum Atmospheres Inc.). These steps assured an initially dry solution, and hydrolysis should then occur only through moisure absorbed by the solution upon exposure to the air and the substrate during the deposition step.

Thirdly, the type of structure obtained is highly dependent on the functionality of the monomer ${ }^{7}$. By blocking a percentage of the polymerization sites, the functionality is effectively reduced and a more open structure is obtained. Thus, upon the addition of a modifying ligand such as DEA, not only is the alkoxide stabilized in solution, but when the gel is formed the structure is less highly branched, and polymerization is uniform on a finer scale. 
As can be seen in Figure 2.1, films derived from sol-gel processing in which these measures were taken appeared fully dense at the limit of SEM resolution (approximately $10 \mathrm{~nm}$ ). Firing temperatures necessary for the formation of crystalline products were as low as $400 \mathrm{C}$, as described below.

\section{Solvent Evaporation and Bumout of Organics}

The initial portions of the heat treatment are important when considering shrinkage stresses that develop within the film and subsequent cracking behavior. The drying schedule and the effect of burnout on stress will be discussed in Chapter 4. In the present chapter, only the effect of the heating schedule on the crystalline phases that form will be discussed. Because the speed of the burnout heat treatment is limited mainly by cracking considerations, no experiments were done to test the limit of quick heating in terms of its effect on the final structure and composition of the final product. The normal schedule consisted of a heating rate of $0.3 \mathrm{C} / \mathrm{min}$ through the low temperature range $(200$ to $400 \mathrm{C})$ in which burnout is expected. From the $\mathrm{X}$-ray data below we can affirm that this rate was slow enough to allow the trouble-free elimination of organic materials. While there should be some concern about interstitial carbon remaining in the ceramic materials, the most effective way to ascertain the presence of carbon would be to detect its influence on electronic properties through the use of impedence spectroscopy, which will not be discussed in this dissertation. In general, carbide decomposition products would not be expected from alkoxides of the more electropositive transition metals, which have strong metal-oxygen bonds.

\section{C: Firing and Crystalline Phase Formation}

Coating solutions were prepared for X-ray diffraction study from isopropoxides of the desired metals. Films were dip-coated on single-crystal silicon 
(111) substrates which had slightly off-axis orientations; a single weak X-ray peak from the substrate can be seen on the patterns described below. (The orientation of the silicon is assumed not to have any effect on the formation of the zirconia film; no epitaxial growth is expected.) Heat treatments were done in air with a $0.3 \mathrm{C} / \mathrm{min}$ ramp to the final temperature, which was maintained for two hours.

Numerous workers have shown that crystalline phases can be formed from zirconium alkoxide solutions at temperatures as low as $400 \mathrm{C} 8,9,10$. In the present study, weak X-ray diffraction peaks were found after a $400 \mathrm{C}$ treatment, with progressively sharper peaks after $600 \mathrm{C}$ and $1000 \mathrm{C}$ treatments. Weak XRD peaks after the $400 \mathrm{C}$ treatment suggests that the crystallite size is quite small (bordering on amorphous), while after the higher temperature treatments it is clear that crystal growth has occured. Indirect evidence (described below) indicates that the crystallite size is still less than about $50 \mathrm{~nm}$ after the $1000 \mathrm{C}$ treatment, which agrees with size determinations from previous studies 11 . While this trend was observed for all samples, the particular phase formed had a more complicated dependence on temperature and on the composition of the starting solution.

\section{Undoped Zirconium Isopropoxide Solutions}

Pure zirconium isopropoxide solutions in isopropanol were prepared for comparison with yttrium-doped solutions. X-ray patterns after 400.600 , and $1000 \mathrm{C}$ heat treatments are shown in Figures 2.2, 2.3, and 2.4, respectively. The samples which were treated at $400 \mathrm{C}$ show only diffuse cubic zirconia peaks, while the $600 \mathrm{C}$ samples still had predominantly the cubic structure, but with distinct monoclinic peaks also present. The samples which were heated to $1000 \mathrm{C}$ were almost completely monoclinic, with some cubic phase remaining. These results do not agree with the equilibrium behavior of pure zirconia, which should be monoclinic below $1170 \mathrm{C}$ and tetragonal above that temperature, with the equilibrium cubic 
phase appearing only above $2370 \mathrm{C}$. The presence of cubic X-ray peaks at low temperatures can be explained using the following information from the literature:

Livage et al ${ }^{12}$ have proposed that the amorphous zirconia which forms at low temperatures (approximately $200 \mathrm{C}$ ) after burnout of organic material actually has preferred interatomic spacings which are similar those of the tetragonal form; amorphous zirconia may actually contain regions which could be considered to be small plates of zirconium atoms between planes of oxygen atoms. These regions could act as nuclei for tetragonal crystallites, which should then be the first crystalline phase to form at low temperatures, assuming no monoclinic nuclei exist. In fact, the tetragonal phase has been found commonly in chemically-derived zirconia powders $12,13,14$, and normally is found to transform to the monoclinic phase at higher temperatures. In the present work, monoclinic indeed becomes dominant after the higher temperature heat treatments, but the low temperature treatments give cubic, rather than tetragonal, $\mathrm{X}$-ray peaks. While a metastable cubic phase has been proposed at such temperatures ${ }^{15}$, the discrepency may also be accounted for by the observation that for very small crystal sizes (approximately 10 $\mathrm{nm}$ ) tetragonal cystals have been found to give rise to only cubic X-ray peaks ${ }^{13}$. (In that study, selected area diffraction patterns were used to demonstrate that such crystals were actually tetragonal.) Thus, the results obtained here suggest that very small crystallites of metastable tetragonal zirconia are present, some of which persist even after a $1000 \mathrm{C}$ treatment in which most of the material has transformed to the stable monoclinic phase.

\section{Yttrium-doped Zirconium Isopropoxide Solutions}

The zirconia-yttria phase diagram, shown in Figure 2.5, predicts stabilization of the cubic phase at a composition of 18 mole percent $\mathrm{YO}_{1.5}$. Solutions which were doped with yttrium isopropoxide to this target composition gave $\mathrm{X}$-ray patterns 
which only contained peaks from the cubic phase; no monoclinic X-ray peaks were observed even for the samples which were treated at $1000 \mathrm{C}$. Figures 2.6, 2.7, and 2.8 show patterns for $400 \mathrm{C}, 600 \mathrm{C}$, and $1000 \mathrm{C}$ samples, respectively. While there is the possibility, as discussed above, that these cubic peaks may have arisen from fine tetragonal crystallites (especially at the lower temperatures), the lack of any monoclinic phase formation suggests that the cubic phase was actually stable as a result of the yttria addition.

These solutions also contained DEA which was added as a modifying ligand at a DEA:Zr molar ratio of 2:1. No evidence of a carbide phase is present on the Xray patterns, suggesting successful burnout of organic materials. Isopropanol solutions containing $0.2 \mathrm{M} \mathrm{DEA}, 0.082 \mathrm{M}$ zirconium isopropoxide, and $0.018 \mathrm{M}$ yttrium isopropoxide were used as the standard $18 \%$ yttria-stabilized zirconia (YSZ) coating solutions in the remainder of this research.

\section{Multicomponent Oxide Formation}

The formation of strontium cerium oxide was attempted using starting solutions of the appropriate isopropoxides. The two metal alkoxides were mixed in an $\mathrm{Sr}$ :Ce ratio of 1:1, and sol-gel films were deposited using the same procedure as for the zirconia films. $\mathrm{X}$-ray diffraction revealed that only $\mathrm{CeO}_{2}$ had formed, along with a material which gave rise to unidentified diffraction peaks. The two metal alkoxides were then studied separately to determine their relative hydrolysis rates (more concentrated solutions $(1 \mathrm{M})$ were used and excess water was added to speed the gelation process). Cerium isopropoxide was found to form a gel much faster than strontium isopropoxide under identical conditions, and it was concluded that additional steps such as refluxing would be needed to insure that the metals remained well-mixed throughout the process. 


\section{D: Substrate Reactions}

Successful formation of the stabilized zirconia phase was discussed above for experiments in which silicon was the substrate. In actual devices the substrate must fulfill certain functions (as discussed in section 1G), and in general the sol-gel film will be in contact with more reactive materials during the heat treatment and during operation of the device. Other substrate materials were tested with the intention of more closely approximating the surroundings which a sol-gel film would encounter in a real device. Results are discussed below.

\section{Metal Substrates}

When a gold layer was sputtered onto a fused quartz substrate prior to solgel film deposition, zirconia formation appeared to be unaffected. Figure 2.9 shows the $\mathrm{X}$-ray pattern; gold diffraction peaks are still present, indicating that the gold layer remained intact and did not react with the other materials. The results were also good for zirconia films deposited on platinum sheeting (Figure 2.10). However, more reactive metals such as nickel were seen to present complications.

Polished nickel substrates were found to oxidize during the heat treatment even when covered by sol-gel films. Resulting X-ray patterns (Figure 2.11) demonstrated that zirconia was unable to form in these conditions; the zirconiurn was apparently incorporated into a much thicker nickel oxide layer which grew during the heat treatment, leaving only nickel oxide peaks. In such situations, it may be possible to control the atmosphere during the heat treatment at a slightly reducing condition to make the formation of nickel oxide thermodynamically unfavorable, while the more electropositive zirconium would still retain its oxygen bonds, giving the oxide.

It was found that repeated sol-gel film deposition over the already-fired sample allowed zirconia to form (Figure 2.12). Apparently, after nickel oxide has 
already formed, it provides an unreactive substrate for zirconia deposition. The success of this method may be due to the fortunate choice of yttria as the cubic stabilizer for the zirconia; it has been shown ${ }^{16}$ that nickel oxide forms a stable interface with yttria-stabilized zirconia, presumably due to the low solubility of yttria in nickel oxide, whereas when calcia or magnesia are chosen as the stabilizer, reaction takes place at the interface. (These compounds have a higher solubility in nickel oxide.)

\section{Alumina Substrates}

The controlled-porosity alumina membranes described in section $1 \mathrm{G}$ were also used as substrates for X-ray diffraction tests. While no reaction between the coating and alumina substrates was found at any stage of the process, some evidence was suggestive of changes in the structure of the alumina itself during the heat treatment.

The membranes give what appears to be a completely amorphous pattern if the diffraction spectrum is taken with the surface of the membrane parallel to the diffracting plane; however, if the membranes are ground with a mortar and pestle and the resulting powder is tested, diffraction peaks can be seen. This result suggests either that the top surface of the membranes is actually amorphous, with crystalline material only found deeper inside the membrane, or that the orientation of the porosity at the surface is such that very little surface area is oriented in the diffracting plane, so that no diffraction peaks are seen until a powder test is done.

In any event, the as-supplied membranes appear to be $\theta$-alumina (Figure 2.13) although unidentified diffraction peaks are also present, and a significant amorphous background can be seen as well. After a $600 \mathrm{C}$ heat treatment, the $\theta-$ alumina diffraction peaks appear unchanged, but the amorphous background appears with less intensity. Thus, the as-supplied membranes, which are formed at 
room temperature from aluminum metal, could contain significant amounts of amorphous material which may begin to crystallize during heating. It is also noted that coated membranes have a tendency to curl up during 900 or $1000 \mathrm{C}$ heat treatments (initially flat disc membranes " snd toward a section of a cylindrical surface), presumably under the action of a tensile stress caused by the shrinking gel. Since the curvature persists even when the sol-gel film is highly cracked, it is assumed to be plastic deformation (an assumption which is in agreement with the fact that the curvature is much greater than can be elastically induced in as-supplied membranes). This permanent deformation may indicate that a significant amount of amorphous material is present in the membranes, as crystalline alumina would not be expected to creep noticeably at such temperatures.

\section{E: Summary of Results}

Crystallization behavior of zirconia precursor solutions was studied using Xray diffraction. Samples were fired at a slow heating rate $(0.3 \mathrm{C} / \mathrm{min})$ to burn off residual organics and were held for two hours in air at $400 \mathrm{C}, 600 \mathrm{C}$, or $1000 \mathrm{C}$. All samples showed slight crystallization at $400 \mathrm{C}$, with progressively sharper peaks at higher temperatures.

Pure solutions of zirconium isopropoxide in isopropanol resulted in films with weak cubic peaks at $400 \mathrm{C}$ and films with progressively higher proportions of monoclinic zirconia at the higher holding temperatures. Solvtions to which yttrium isopropoxide was added (for a target composition of 9 mole percent $\mathrm{Y}_{2} \mathrm{O}_{3}$ ) produced films with only cubic peaks at all holding temperatures. Diethanolamine (DEA) was also added to yttrium-containing solutions in various amounts to act as a blocking ligand which would prevent three-dimensional polymerization or precipitation. No alteration of the cubic peak positions was seen for these mixtures, and no unidentified peaks are present. 
The stabilized zirconia films which are formed from this technique appear fully dense, with a very small crystallite size $(10-50 \mathrm{~nm})$, indicating that the control of polymerization to produce fine structures was effective.

No film/substrate reactions were detected when ceramic materials were used as the substrate; however, when coating was attempted on nickel, the growth of a nickel oxide layer prevented a zirconia film from forming. The nickel oxide layer was found to provide an unreactive substrate upon successive coatings. Nobler metals also appear to provide unreactive substrates. Using these results, anticipated compatibility requirements for actual device operation will be discussed in Chapter 5.

\section{References--Chapter 2}

1 Y. Takahashi and Y. Matsuoka, "Dip-Coating of $\mathrm{TiO}_{2}$ Films Using a Sol Derived from Ti(O-i-Pr) 4-diethanolamine-H2O $_{2} \mathrm{O}-i$-PrOH System", J. Mat. Sci. 23 2259-2266 (1988).

2 B. Yoldas, "Zirconium Oxides Formed by Hydrolytic Condensation of Alkoxides and Parameters that Affect their Morphology", J. Mat Sci. 21, 1080-1086 (1986).

3 Chung-Hsin Lu, private communication.

4 D. C. Bradley, R. C. Mehrotra, and D. P. Gaur, Metal Alkoxides, p. 302 (Academic, London 1978).

5 Steinhauser, M., unpublished work.

6 D. C. Bradley, R. C. Mehrotra, and D. P. Gaur, Metal Alkoxides, p. 318 (Academic, London 1978).

7 C. Brinker and G. Sherer, Sol Gel Science, p. 797 (Academic, Boston 1990).

8 E. Tani, M. Yoshimura, and S. Somiya, "Formation of Ultrafine Tetragonal $\mathrm{ZrO}_{2}$ Powder Under Hydrothermal Conditions", J. Amer. Cer. Soc., 66 [1], 11 (1983). 
${ }^{9}$ K. S. Mazdiyasni, C. T. Lynch, and J. S. Smith, "Preparation of Ultra-High Purity Submicron Refractory Oxides", J. Amer. Cer. Soc., 48 [7], $372-75$ (1965).

10 K. Izumi, M. Murakami, T. Deguchi, A. Morita, N. Tohge, and T. Minami, "Zirconia Coating on Stainless Steel Sheets from Organozirconium Compounds", J. Amer. Cer. Soc., 72 [8], 1465-68 (1989).

11 P-Y. Chu and R. C. Buchanan, "Characteristics of Oxide Thin Films from Carboxylate Precursors", J. Mater. Res., 6 [8], 1736-43 (1991).

12 J. Livage, K. Doi, and C. Mazieres, "Nature and Thermal Evolution of Amorphous Hydrated Zirconium Oxide", J. Amer. Cer. Soc., 51 [6], 346-53 (1968).

13 T. Mitsuhasi, M. Ichihara, and U. Tatsuke, "Characterization and Stabilization of Metastable Tetragonal Zirconia", J. Amer. Cer. Soc., 57 [2], 97 (1974)

${ }^{14}$ E. Tani, M. Yoshimura, and S. Somiya, "Formation of Ultrafine Tetragonal Zirconia Powder Under Hydrothermal Conditions", J. Amer. Cer. Soc., 66 [1], 11 (1983)

15 K. S. Mazdiyasni, C. T. Lynch, and J. S. Smith, "Metastable Transitions of Zirconium Oxide Obtained from Decomposition of Alkoxides", J. Amer. Cer. Soc., 49 [5], 286-7 (1966).

16 S. L. Shinde, I. E. Remainis, and L. C. DeJonghe, "Evolution of the Nickel/Zirconia Interface", Cer. Eng. and Sci. Proc. 7 [7-8] (Am. Cer. Soc., 1986). 


\section{Chapter 3: Effects of Substrate Porosity During Deposition Step}

This section is concerned with the deposition step of the sol-gel process, and how deposition can be accomplished on porous substrates. A review of the available deposition techniques is presented first, followed by a description of the deposition procedures used in this research. Deposition on porous substrates under wetting conditions is discussed next, and the results of these experiments are presented.

\section{A: Deposition Techniques}

There are several ways of depositing a thin film from a bulk liquid-ofeatures common to all techniques include a dependence on the viscosity of the liquid and a way of controlling the thickness to a uniform value.

\section{Dip Coating}

Dip coating is probably the simplest coating technique to perform, as it consists of submerging a substrate into the solution and then withdrawing it at a controlled speed and angle. The thickness of the film, $t_{p}$,depends on these parameters as well as on the viscosity of the solution, its density, and its surface tension ${ }^{1}$ :

$$
t_{p}=J\left[\frac{\rho-\rho_{s}}{\rho_{p}-\rho_{s}}\right]\left[\frac{\eta U}{\rho g}\right]^{1 / 2}
$$

$\mathrm{J}$ is a dimensionless flow term approximately equal to $0.2, \rho$ is the solution density, $\rho_{\mathrm{p}}$ and $\rho_{\mathrm{S}}$ are densities of the film and solvent, respectively, $\eta$ is the solution viscosity, $U$ the withdrawal speed, and $g$ is the acceleration of gravity.

The uniformity of the thickness is quite good for this technique when large areas are coated (it is used in industrial processes such as reflective coating of 
windows ${ }^{2}$ ). Limitations in uniformity arise chiefly from edge effects and also from very slow withdrawal speeds 3 . Films tend to be thicker near the sides of the sample due to the formation of a meniscus. There is also a tendency to form a parabolic thickness distribution (increasing thickness from the top edge of the sample to the bottom), arising from flow in response to gravity. However, such thickness variations are in reality limited to a characteristic distance from the sample ends which depends on solution properties ${ }^{4}$. In the present work, these edge effects were only seen within a few millimeters of the edges of the sample. Film irregularities may also arise in dip coating due to contamination of the solution, turbulence, or local changes in air flow. Clean rooms and rigorous control of air flow are needed for uniform coating of large areas.

An added advantage of dip coating for the purposes of this research is the ability to vary the thickness over the length of a single sample through accelerating the sample during withdrawal. In this way the effect of film thicknesses over a continuous range can be easily observed (see Chapter 4).

\section{Spin Coating}

Spin coating is the preferred technique for coating flat circular samples; it is widely used in the semiconductor industry for coating wafers with photoresist. The substrate is usually mounted by a vacuum chuck to a motor, which can be accelerated at high rates after an excess of coating solution is deposited in the center of the sample.

Film thickness is determined basically by a balance between centrifugal force and the frictional force from the substrate. Spin speed and viscosity are thus the two main factors in determining film thickness. The force balance has been calculated ${ }^{5}$, giving the following dependence of the film thickness $\mathrm{h}$ : 


$$
h \propto f^{-2 / 3} \eta^{1 / 3} e^{1 / 3}
$$

where $f$ is the spin speed, $\eta$ is the viscosity, and $e$ is the evaporation rate. The evaporation rate in turn is proposed to be proportional to the square root of the spin speed, giving the spin speed dependence as

$$
h \propto f^{-1 / 2}
$$

However, the actual description of the coating behavior is more complicated, due to the actions of other forces (such as frictional forces due to air flow), differences betwien the forces acting on the center and outer regions of the sample, and changes in behavior as the different stages of deposition occur (acceleration, spin-off of excess fluid, and evaporation) ${ }^{4}$. An empirical relationship based on some simple pararneters is most easily used for determining film thickness 6 :

$$
h=\frac{100 p^{2}}{\sqrt{f}}
$$

where $h$ is the film thickness in angstroms, $p$ is the percent solids in the coating solution, and $f$ is the spin speed in thousands of revolutions per minute. This equation was found to be adequate for predicting the film thickness in the present work.

Spin coating differs from dip coating in the important respect that a strong shearing force can be applied to the thinning film. It has been reported that by increasing the spin speed (and hence the shearing force), changes in the structure of the gel obtained can be dramatic; the index of refraction increases with increasing speed, and the radial alignment of polymers has been detected 7 . Clearly, the spin coating process allows some control over the winy that polymers pack together during consolidation. 


\section{Other Film-Forming Techniques}

A thin membrane of ceramic material can be formed by other methods as well. Conventional techniques such as doctor-blade spreading, tape casting, or spray-coating may be applicable, but in most cases will give rise to a membrane which is too thick for electrolyte device applications. As discussed in section $3 \mathrm{C}$, a modified spin coating method using a volatile substrate, as well as a wire-ring drawing method, can be used to produce unsupported films. However, conventional dip-coating and spin-coating appear to be capable of producing the films with the most desirable properties.

\section{B: Coating Step Experimental Procedure}

Both dip-coating and spin-coating were utilized in this work. Investigation of the sol-gel chemistry and crystalline phase formation was done using dip-coated silicon wafers, while a variety of substrates and both coating methods were used to study cracking behavior and the effect of substrate porosity.

All substrates were rinsed in acetone and alcohol prior to deposition. In addition, silicon substrates in some cases were rinsed in dilute nitric acid and/or given a $900 \mathrm{C}$ heat treatment in air prior to deposition to test the effect of these cleaning efforts on film adhesion.

\section{Dip-Coating}

Submerged samples were pulled up at varying speeds (from roughly 5 $\mathrm{cm} / \mathrm{min}$ to $100 \mathrm{~cm} / \mathrm{min}$ ). For the faster pull rates, the samples were simply pulled out by hand with tweezers, while slower pull rates were accomplished by hanging the sample from the short end of a lever (to allow faster hand movement when gripping the lever at the longer end). No attempt was made to achieve a perfectly uniform coating over large areas using dip-coating, as adequate uniform areas could always 
be found during microscopy. In some cases the pull rate was deliberately varied on a single sample in order to observe the effect of continuous thickness changes.

\section{Spin-Coating}

A vacuum-chuck spin-coater (Integrated Technologies) was used; substrates which lacked rigidity were fastened to disc supports using double-sided tape in order to withstand the pull of the vacuum. Coating solutions were pipetted into a small pool in the center of the sample before the coater was turned on. The samples were then rapidly accelerated $(2000 \mathrm{rpm} / \mathrm{sec})$ to speeds of 2000 to $8000 \mathrm{rpm}$, spinning off excess droplets of solution. This technique gave quite uniform thicknesses over large areas.

Heat treatments were then performed as described in Chapter 2.

\section{C: Wetting of Pores During the Deposition Step}

The description of deposition methods at the beginning of this chapter made no mention of how a porous substrate would affect the deposition step. If a thin, flat film is desired on top of a substrate with completely open porosity, then the deposition step presents a problem. The behavior of the coating solution clearly depends on the wetting angle for the substrate/solution/atmosphere triple interface.

A solution which does not wet the substrate would be expected to remain completely above the substrate if the pores had sufficiently small diameters; thus, the first course of action might be to find a system with a favorable wetting angle, or to alter the surface energy of the substrate with a pre-coating. However, even if such a system were found, wetting is generally necessary for the sol-gel film coating process if good adhesion is desired. Therefore, other strategies must be considered. 


\section{Possible Remedies for Pore Wetting}

If the coating liquid must have a tendency to enter substrate pores, then one possible strategy is to postpone contact between the liquid and the substrate until the film gains some rigidity. Free casting of unsupported films represents a possible solution to the wetting problem (and more importantly, solves the problem of shrinkage cracking due to a substrate constraint), but such a process is difficult, and formation of large-area uniform thin films is nearly impossible. Sakka et al.

describe a wire-ring drawing process for forming a completely free film ${ }^{8}$, but report problems with uneven film thickness or breakup of the film, depending on the solution viscosity.

Unsupported films formed on volatile substrates have also been reported 9 . Polished camphor discs were used as spin-coating substrates and were allowed to volatilize afterward, producing free films. However, volatilization of the camphor before coating was completed caused roughness in the surface of the film, and severe warpage of the films during drying was reported; the authors conclude that such a process is useful as a fast characterization method of the sol-gel process, but not for the production of high-quality films.

A possible variation of the above method might consist of impregnating a porous substrate with a volatile filler material, so that upon firing the film would come into contact with the rigid substrate, thereby possibly preventing warpage and allowing some adhesion to the substrate. That approach was not attempted in this research, due to uncertainty in how adhesion between the film and substrate might be obtained, as well as the apparent difficulty in obtaining a smooth surface on which to coat. While these problems might be overcome eventually, in the current work a simpler approach was adopted, as described in section 3D below.

Another alternative to consider is the use of particulate sols rather than polymeric solutions, so that if the particle size is larger than the size of pores in the 
substrate, the coating will remain above the substrate. However, such a process would eliminate some of the key advantages of the sol-gel process; films formed in such a way would undoubtedly be thicker and more porous than films formed from polymeric gels, and sintering temperatures would have to be increased. The utility of such a process is probably limited to the creation of a substrate/electrode with graded porosity (which would be desirable for electrolyte devices); these ideas will be discussed in Chapter 5 .

\section{Rapid Coating}

If a substrate/coating solution system is chosen which has complete wetting, there remains the possibility of rapidly spin-coating a solution to dryness before the liquid has a chance to fill the pores of the substrate. To determine the necessary speed for such a process, the pores can be modeled as cylindrical capillaries, and viscous flow rates can be calculated.

Poiseuille's law for viscous flow through a cylindrical capillary under a differential pressure $P$ is

$$
\frac{d V}{d t}=\frac{\pi \mathrm{Pr}^{4}}{8 \ln }
$$

where $\mathrm{V}$ is the volume of solution, $\mathrm{r}$ and $l$ are the radius and length of the cylinder, and $\eta$ is the viscosity of the solution. We wish to consider the time required to wet a pore of a characteristic length; therefore using $\mathrm{V}=\pi \mathrm{r}^{2} l$ we have

$$
\frac{d l}{d t}=\frac{\operatorname{Pr}^{2}}{8 \ln }
$$

Integration yields the time to wet a pore of length $l$ :

$$
t=\frac{4 l^{2} \eta}{\mathrm{Pr}^{2}}
$$


This equation can be modified by using the capillary pressure, $2 \gamma / \mathrm{r}$, in place of the pressure $\mathbf{P}$, where $\gamma$ is the surface tension of the liquid and complete wetting is assumed:

$$
t=\frac{2 l^{2} \eta}{\gamma r}
$$

It is important to note that while capillary pressure increases for smaller pores, wetting speed in fact decreases for smaller pores, due to the stronger dependence of viscous flow on pore size. Characteristic pore wetting times can now be estimated for isopropanol solutions deposited on controlled-porosity substrates, using $\eta=0.02$ dyne-s $/ \mathrm{cm}^{2}$, and $\gamma=22 \mathrm{dyne} / \mathrm{cm}$, the values for pure isopropanol. According to equation 3.8, Anotec membranes with columnar 0.2 micron diameter porosity will be saturated completely through their 50 micron thickness in 4.5 milliseconds. Obviously, much longer characteristic times are needed if pore filling is to be avoided in a normal spin coating process. Even with the use of a 0.02 micron porosity membrane, the time cannot be lengthened by more than a factor of ten. More drastic changes in either $r, b \quad \gamma$, or $\eta$ would be needed before a rapid coating technique could be effective for the prevention of pore wetting; changing these parameters would undoubtedly upset other aspects of the sol-gel process.

\section{D: The Filled-Pore Coating Process}

The liquid film when it is first deposited on the substrate has a much greater thickness than the film in its final, fired state. The shrinkage has important consequences; the major disadvantage resulting from shrinkage is film cracking (see Chapter 4), while a possible advantage of large shrinkage lies in the ability to completely fill substrate pores with liquid during the initial deposition, but still obtain free porosity of the substrate after consolidation of the liquid into the final, dense ceramic material. 
The coating solutions which gave the optimum films in this research consisted of $0.082 \mathrm{M}$ solutions of zirconium isopropoxide in isopropanol, together with $0.18 \mathrm{M}$ yttrium isopropoxide and $0.2 \mathrm{M}$ diethanolamine (DEA). The equivalent weight of $\mathrm{ZrO}_{2}$ in a $0.1 \mathrm{M}$ solution of the metal is $0.0123 \mathrm{~g} / \mathrm{cm}^{3}$. If fully dense zirconia (theoretical density $=5.9 \mathrm{~g} / \mathrm{cm}^{3}$ ) is the final product, then the final volume of zirconia is $0.21 \%$ of the initial liquid volume.

Due to this low zirconia content in the coating solution, the final volume of zirconia formed in any pore will be less than $1 \%$ of the pore volume. If uniform coating of the pore walls is assumed, then the thickness, $t$, of the final pore-wall coating can be calculated from volume conservation:

$$
t=\frac{\pi r^{2}}{2 \pi r} \times 0.21 \%=0.001 r
$$

Sub-micron pores that have initially been filled with solution would then be expected to have only a discontinuous film of zirconia on their walls (sub-nanometer films would not be expected to remain continuous if they form at all), and the gas/electrolyte/substrate triple interface should remain at the surface of the substrate. Thus, the functioning of a fuel cell or related device would be unaffected by zirconia within the substrate porosity; pore sizes would have to be 10 microns or larger before continuous internal zirconia films would be a concern.

\section{Solution Puddling}

Allowing the pores to fill with coating solution, of course, means that the substrates should be thin, so that the total volume of solution drawn into the pores is not too great (unless one is willing to waste large quantities of coating solution). An excess of coating solution must always be used to insure that an adequate puddle remains on top of the substrate. 
Drainage of the coating solution completely through the substrate (with leakage out of the bottom) must also be considered. Such drainage after the substrate is saturated would not be driven by capillary pressure, but only by gravity. Equation 3.5 can then be used to determine the flow rate, with the quantity $\rho$ gh substituted for the pressure $P$, where $\rho$ is the density of the coating solution, $g$ is the acceleration of gravity, and $h$ is the depth of the puddle:

$$
\frac{d V}{d t}=\frac{\pi \rho g h r^{4}}{8 \ln }
$$

This flow rate is for a single capillary of radius $r$; to determine how the puddle depth $\mathrm{h}$ changes, we can assume a $50 \%$ porosity substrate, such that the surface area drained per capillary is $2 \pi r^{2}$. We then have

$$
d V=2 \pi r^{2} d h
$$

where $\mathrm{V}$ is now the volume drained per capillary. Equation 3.10 then becomes

$$
\frac{d h}{d t}=\frac{\rho g h r^{2}}{16 l \eta}
$$

Integration from $h_{1}$ to $h_{2}$ yields:

$$
t=\frac{16 \ln \eta}{\rho g r^{2}} \ln \frac{h_{1}}{h_{2}}
$$

We can now determine a characteristic time for the drainage process. Using the isopropanol values for the constants, and using the dimensions of a 0.2 micron pore Anotec membrane for the $r$ and $l$ values, the calculated time for a puddle to drain half its height $\left(h_{1}=2 h_{2}\right)$ is more than four hours. There need be no concern about drainage through the substrate until this characteristic time approaches about 10 seconds; the size of pore which would have that characteristic time is more than 30 microns. We can conclude that the pore-filling process will give adequately stable puddles for all substrates of interest. 


\section{Results of the Filled-Pore Method}

Fracture cross sections of different coated substrates have been examined to determine the extent of zirconia formation within the pores.

As expected, the success of film formation on porous substrates depended on the scale of the porosity. Thick substrates with large pores absorbed large volumes of coating solution, leaving no continuous liquid film on the surface but causing unwanted zirconia formation deep within the pores. This type of coating behavior was observed for substrates produced by tape casting or bulk sintering. Such methods are currently in use to produce porous substrates which are being considered for solid electrolyte devices. Sintered $\mathrm{La}_{0.6} \mathrm{Sr}_{0.4} \mathrm{MnO}_{3}$ (LSM), which is one such substrate, is shown in Figure 3.1; surface features and porosity on a scale of 5 to 10 microns can be seen.

Since these tape-cast substrates were quite thick (more than a millimeter in some cases), they were able to absorb all of the coating solution applied in the usual amount. No diffraction peaks for zirconia were observed on coated LSM substrates, and under SEM observation the surface appeared unchanged from its pre-coated condition. While it would be interesting to apply enough coating solution to saturate the entire substrate in the hopes of forming a surface film, such an attempt was not made. (As will be discussed in Chapter 4, severe cracking would take place on a substrate with such large porosity anyway.)

Additional coating experiments were done on Anotec membranes with uniform pore sizes of 0.2 and 0.02 microns. The surface of a 0.2 micron pore size membrane is shown in Figure 3.2. As predicted by the discussion above, both sizes of coated Anotec membranes allowed zirconia to form at the surface (confirmed by XRD and SEM). The pores appeared free of any unwanted zirconia formation under SEM viewing of fracture cross-sections of the coated subsirates (Figure 3.3). 


\section{E: Summary of Coating Step Results}

It was determined that wetting and saturation of the substrate pores cannot realistically be avoided without drastically altering the nature and adhesion of the film. However, a method of coating in which the substrate pores are allowed to fill with coating solution was determined to be useful if the following conditions are met: i) The substrates are thin, so that the total pore volume is small. ii) The size of the pores is less than about 10 microns, so that continuous internal films on the pore walls are avoided, and also so that the pool of coating solution will remain on top of the substrate for a reasonable length of time while the spin-coater is being accelerated.

Experimental results show that films can be successfully formed on porous alumina membranes when those conditions are met.

\section{References---Chapter 3}

1 I. Strawbridge and P. F. James, "Thin Silica Films Prepared by Dip Coating", J. Non-Cryst. Solids 82 366-372, (1986).

2 H. Dislich in Sol-Gel Technology for Thin Films, Fibers, Preforms, Electronics and Specialty Shapes, p. 50, ed. L. C. Klein (Noyes, Park Ridge NJ, 1988).

3 I. Strawbridge and P. F. James, "The Factors Affecting the Thickness of Sol-Gel Derived Silica Coatings Prepared by Dipping," J. Non-Cryst. Solids, 86 381-393 (1986).

4 L. E. Scriven, "Phsics and Applications of Dip Coating and Spin Coating", pp. 717729 in Materials Res. Symp. Proc. Vol. 121, ed. C. J. Brinker, D. E. Clark, and D. R. Ulrich, Mat. Res. Soc., 1988.

5 D. Meyerhofer, "Characteristics of Resist Films Produced by Spinning", J. Appl. Phys. 49 [7] 3993-97 (1978).

6 R. A. Colclaser, Microelectronics: Processing and Device Design, p. 40 (Wiley, New York, 1980). 
7 C. J. Brinker, A. J. Hurd, and K. J. Ward, "Fundamentals of Sol-Gel Thin-Film Formations", p. 223 in Ultrastructure Processing of Advanced Ceramics, ed J. Mackenzie and D. Ulrich (Wiley, New York 1988).

8 S. Sakka, K. Kamiya, K. Makita, and Y. Yamamoto, "Formation of Sheets and Coating Films From Alkoxide Solutions," J. Non-Cryst. Solids, 63 223-235 (1984).

9 J. C. Huling and G. L. Messing, "A Method for Preparation of Unsupported SolGel Thin Films", J. Am. Cer. Soc. 71 [4] C-222-C-224 (1988). 


\section{Chapter 4: Mechanical Behavior of Films During Processing}

This section deals with a description and discussion of the cracking behavior of the sol-gel derived films which were studied in this research. Some background on shrinkage cracking is presented first. The observed cracking behavior on smooth, continuous substrates is then described, and processing strategies which were used for crack-free formation of films on these substrates are discussed. Following this are the results obtained when porous substrates were used, and finally a theoretical discussion of the mechanisms of cracking is presented.

\section{A: Background: Shrinkage Cracking}

Standard sol-gel techniques for the formation of oxide ceramics involve reactions in which a large percentage of the products are not used in the final product. During the transformation, then, from a liquid coating to the final crystalline product, a significant shrinkage occurs which is resisted by the constraint of the substrate. While much of the shrinkage will take place during solvent evaporation when the material is still able to flow to relieve stresses, significant strain will also accompany burnout of organics and crystallization. The increasing film rigidity will then lead to residual stresses, and in some cases crack formation. In this research, conditions have been varied to determine when shrinkage cracking can be avoided.

\section{Altering Polymerization to Reduce Shrinkage Stress}

The extent of hydrolysis and condensation reactions can be controlled by the amount of water added and also by the addition of chelating ligands, which can block hydrolysis sites. Three-dimensional polymerization (leading to a rigid gel) may be avoided by leaving an average of only two hydrolysis sites per metal atom unblocked; in this way a linear polymeric structure may be the favored product, 
which would have important consequences for the flow properties of the gel 1 . In addition to reducing the viscosity of the gel, the selective blocking of hydrolysis sites can enable the gel to collapse to a thin film as the solvent evaporates rather than to retain its three-dimensional rigidity. In this way most of the shrinkage will take place normal to the plane of the substrate, leaving the film in a relatively unstressed condition. Rapid solvent evaporation also improves the collapsibility of the film by reducing the time during which the gel network can age, or create cross links that increase the rigidity.

The network should also be as dense as possible after solvent evaporation, so that shrinkage during burnout is reduced---thus the bulkiness of side groups and blocking ligands should be kept to a minimum. A tradeoff exists between this goal of reducing the weight of side groups, and the ligand extension that is generally necessary to prevent excessive rigidity in the gel. A more complete chemical study would be necessary to determine the optimum composition.

\section{Film Thickness and Crack Driving Force}

By far the most important parameter for the elimination of cracking is the thickness of the film, as described in the literature $2,3,4$. Thick films have more strain energy to release during cracking, and the constraint of the substrate is not as restrictive of relaxation in thick films. In this study, cracking behavior has been examined as a function of the film thickness, which can be controlled by the altering the viscosity of the coating solution or changing the deposition conditions. Adhesion to the substrate also plays an important role in the energy balance that describes unstable cracking, and the effect of varying the substrate characteristics has been observed. 


\section{B: Description of Shrinkage Cracking on Smooth, Continuous Substrates}

Cracking as a result of the heat treaiment was observed consistently in thick films. Initially, coatings on single crystal silicon were used to study the morphology and cracking behavior of the film. The films were observed to be uniform and dense except for sharply defined cracks; no features could be observed within the intact regions of the film at the limiting resolution of the SEM. The cracks are linear and are accompanied by some delamination; the film can be seen to curl up slightly, revealing a channel of bare substrate in the crack path (Figure 4.1). The location of cracks relative to one another appears to be semi-random; that is, cracks occur at random angles and the zirconia "islands" left intact after cracking have a wide size distribution (Figure 4.2). Cracks often end at an intersection with a second crack at a random angle. 'This arrangement is most easily interpreted as revealing the sequence in which the cracks formed.

While the random occurrence of cracks leads to a wide distribution of island sizes, it was observed that the mean island size increased in proportion to the film thickness, that is, the frequency of crack occurrence decreases as the film thickness increases.

A second coating was deposited over some of the cracked films in an effort to create a continuous ceramic layer. As can be seen in Figure 4.3, the second coating left a pattern of film islands on top of the original islands, while within the channels of bare substrate the second coating fractured more severely, leaving small segments of material lined up in the channels. While the overall effect may be aesthetically pleasing, it is noted that bare substrate can still be seen, rendering this structure useless as an electrolyte layer.

Experiments with up to four coatings were conducted, but in each case the cracks in the original layer appeared to provide a nucleation site for cracking in all 
subsequent coatings, making through-thickness openings inevitable. Clearly, a better approach is to attempt a crack-free initial coating, as is discussed below.

\section{Critical Film Thickness}

Film thicknesses were determined by viewing cracks in the SEM at high angles ( $65^{\circ}$ from perpendicular to the surface). The critical thickness for stabilized zirconia films on continuous silicon substrates was found to be about 0.5 microns (Figure 4.4); when the coating solution was diluted to produce films thinner than this value no unstable cracking was observed. Cracks could still be seen extending outward from dust particles or other defects, but the energy balance for cracking was clearly unfavorable, as the cracks closed within about 10 microns of the defect (Figure 4.5). Crack-free regions could be seen extending for hundreds of microns between dust particle-induced cracks; clean room processing should eliminate all cracking for films below the critical thickness on continuous substrates.

It was difficult to assess the dependence of the critical thickness value on the type of continuous substrate, due to the low precision of the thickness measurement. No measurable change in the value of the critical thickness was observed when other types of continuous substrates were used (including fused quartz, sputtered gold on quartz, and polished platinum), indicating that the critical thickness was not strongly dependent on the nature of the bonding to the substrate. Substrate treatments such as pre-firing or acid cleaning (as discussed in Chapter 3 ) did not produce measurable changes in the critical thickness value. Since such measures produced slight changes in adhesion, it is expected that more precise measurement of critical film thickness would reveal a slight dependence. The only instance in which the substrate character caused a marked change in the value of the critical thickness was when a $50 \%$ porosity substrate was used, as discussed below. 


\section{Residual Stresses}

While no stress measurements were attempted, some information about residual stresses in the films can be gained from indirect evidence. In the thicker films, where extensive delamination took place after cracking, a definite curvature was observed in the largest remaining flakes (Figure 4.6). The concavity of the surface indicates that residual stresses existed in the film at some stage after cracking occurred, and that those stresses were more intense in the top part of the film. Such a stress distribution has been reported for other systerns as well ${ }^{5}$.

It should be pointed out here that when coatings below the critical thickness (giving uncracked films) are repeated, the resulting thick films remain uncracked, even though the total thickness is much greater than the critical thickness. If the instance of cracking is wholly determined by an energy balance as discussed above, then it can be assumed that the final structure is less highly stressed than was the film during the cracking stage of the heat treatment. However, this argument for stress release at a later stage of heating is probably too simplistic; as discussed in section 4D, defect formation probably plays a key role in determining when the film will crack. A more direct method of measuring residual stresses, such as measuring the curvature of a film/substrate composite structure, would be needed to unambiguously determine the final stress state.

\section{C: Effect of Substrate Porosity on Cracking Behavior}

The alumina membranes described in section $1 G$ were used to investigate the effect of substrate porosity. The pore size in these membranes can be controlled in a narrow size distribution, so that the use of two different filtration sizes $(0.2$ micron and 0.02 micron) allows the effect of pore size to be tested independently. 


\section{Reduction in Critical Thickness}

Cracking similar to that observed for continuous substrates was present in thick films on membranes of both pore sizes (Figures 4.7 and 4.8). Again, limited delamination is seen to occur, revealing the bare substrate between intact film islands. The value of the critical thickness was found to be about 0.2 microns for both sizes of filter; below this thickness the linear cracks of the type found for continuous substrates are absent. This value represents a reduction in critical thickness, indicating that cracking is more favorable when the substrate is porous. Since both membrane pore sizes have the same percentage of porosity, it could be argued that the reduction in critical thickness is determined by the total porosity, not the pore size (See section $4 D$ ).

\section{Delamination Cracking Within the Substrate}

When the porous alumina substrates were used, delamination was seen to a greater extent. Films which were much thicker than the critical value could be seen to delaminate almost completely from the substrate, leaving only a small area in the middle of each island still attached (Figure 4.9). A section of film which was completely separated is shown in Figure 4.10. It can be seen that the delamination crack dove into the substrate as it progressed away from the original shrinkage crack, and indeed part of the aligned porosity of the substrate can be seen attached to the center of the island. This behavior was predicted by Hu and Evans 5 in the case of stable delamination cracking on brittle substrates, and arises because the crack tends to follow the plane at which $\mathrm{K}_{\mathrm{II}}=0$ (meaning there is no driving force for shear in the plane of the crack), which lies within the substrate. At this location, the crack has pure mode I character (cleavage under tension), which is favored in brittle materials. The stresses tend toward zero near the center of the island, which explains why the islands remain attached in the middle. The free chunk in Figure 
4.10 must have resulted from some externally applied stress during the preparation of the sample for microscopy.

\section{Localized Cracking (Pore Induced Cracking)}

While the network of cracks which formed in thick films looked similar to the patterns observed on continuous substrates, when the film thickness was reduced cracks with a different character began to appear on porous substrates. This second type of cracking occurred locally above pore openings; such cracks can be seen for the larger-pore $(0.2$ micron) substrates in Figure 4.11 within film islands left by the larger-scale linear cracking. These localized cracks persist no matter how thin the film is made, tending toward a limit of what appears to be a completely uncoated substrate, which could be thought of as a film with a localized "crack" over every pore.

It should be emphasized that the localized, pore induced cracking is seen only in films thinner than a limiting thickness value, whereas the cornmon shrinkage cracks exist in films thicker than a critical value. A question arises, then, as to whether there exists a thickness between these values at which crack-free films can be made. Figure 4.12 shows a transition zone in a region of varying coating thickness on a 0.2 micron pore size membrane. For this substrate the two types of sracking have overlapping thickness regimes and therefore no choice of film thickness will allow a continuous film to form.

However, when the smaller pore size ( 0.02 micron) membrane is used as the substrate, the pore-induced type of cracking is inhibited in all but the thinnest films; the limiting thickness is greatly reduced. Figure 4.13 shows a thickness transition zone for these finer-scale porosity membranes, demonstrating that unstable cracking ends at a thickness for which pore-opening cracking is not yet favorable. Continuous films are thus possible for these substrates at thicknesses just under 0.2 
microns. The lower limiting thickness for crack-free films was difficult to determine using the SEM; pore-induced cracking on these substrates occurred at film thicknesses near the resolution limit. In any event, the film in Figure 4.13 demonstrates a successful film formation on a $50 \%$ porous substrate, an important step towards arriving at functional electrolyte devices from the sol-gel process.

Pore-induced cracking for these two membranes is seen to occur below a critical film thickness which is, in each case, of the order of the size of the pores. Thus, because of the maximum thickness limit imposed by the onset of unstable cracking for thick films, the pore-induced type of cracking could be considered to impose a maximum limit on the size of the pores allowed for successful coating. In the limit of very fine pores (approaching continuous substrates), films can be made indefinitely thin and still be continuous, until other mechanisms such as diffusional breakup ${ }^{6}$ begin to affect continuity.

\section{D. Theoretical Discussion of Cracking Mechanisms}

\section{Origin of the Critical Thickness}

The linear cracks which are seen for thick films appear identical regardless of the substrate; for this type of cracking a single mechanism should be active whether the substrate has porosity or not.

Cracks must begin at defects or pre-existing cracks which have a critical size that depends on the level of stress in the film: the quantity

$$
K_{l}=f \sigma \sqrt{a}
$$

where $f$ is a factor which depends on the geometry, $\sigma$ is the uniform stress in the material, and $a$ is the crack length, is known as the stress intensity factor. When this factor exceeds a critical value, the fracture toughness of the material, then the crack will propagate:

$$
K_{l}=K_{I_{c}}
$$


The strain energy which is available for release per unit crack length, the driving force for cracking, increases with crack length when the material has no external constraints. Because of this dependence, cracking dependence on film thickness can be understood from a simple energy balance perspective. Unstable cracking will occur when the strain energy released per unit crack length exceeds the energy required to form the new crack surfaces. For an adherent film, regardless of the defect sizes present, $\mathrm{K}_{\mathrm{I}}$ will be limited by the film thickness, as this corresponds to the maximum effective crack length in terms of the strain energy available for release; the constraint of the substrate limits the volume of material in which the stain can be released, regardless of the crack length in the plane of the film (Figure 4.14). Thus, for a given stress state, there is a critical film thickness $h_{c}$ below which unstable cracking will not occur.

$$
K_{l_{c}}=f \sigma \sqrt{h_{c}}
$$

In this case, the geometrical factor $f$ will also depend on the relative stiffnesses of the film and substrate; the factor will be the same as in equation 4.1 if the stiffnesses are equal. Sol-gel films can be expected to have low stiffnesses during the cracking period, so that in general when stiff substrates are used, the f factor will be slightly smaller than it was in equation 4.1 .

If the film is not adherent, that is, if delamination occurs, then a larger amount of strain energy can be released during cracking, and the critical film thickness value may be reduced (Figure 4.15). Films which are deposited on porous substrates will always have areas which can be considered to be non-adherent, so that the tendency to crack in these areas will generally be greater (as discussed below). 


\section{Critical Flaw Size}

The critical thickness $h_{c}$ was derived for the energy balance of a steady-state crack; for such a crack instability can be determined by considering a cross-section of the running crack, which will give the strain energy released per unit crack length, as in Figure 4.14.

However, the initiation of a crack must be considered from a non-steadystate perspective; the sub-critical flaw which is present represents surface energy that is already available, together with a volume in which the strain has already been released. Thus, only the additional energies associated with further flaw growth should be considered to determine the point of instability. For a growing crack in an unconstrained material, the incremental release volume is proportional to the square of the crack length. This means that strain release energy will soon overpower crack surface energy; a critical length will soon be reached at which the energy balance becomes favorable for cracking. Assuming the material relaxes in the same way as it did for the cross-section of a running crack, the critical flaw size is calculated to be exactly half the critical film thickness ${ }^{5}$ (Figure 4.16).

\section{Intercrack Spacing in Thick Films}

An explanation of the crack spacing in films thicker than the critical thickness must agree with two important observations: 1) The mean intercrack spacing increases linearly with film thickness, and 2) There is a large standard deviation of intercrack spacings for any given film thickness. It will be shown here that a modified elastic model can explain both of these observations.

The linear relationship of spacing with thickness is suggestive of many possible mechanisms, such as separations dictated by elastic or plastic relaxations near the cracks. Such mechanisms have been discussed by Atkinson and Guppy 4 ; however, in that discussion no model had any great success in agreeing with the 
observed behavior. Furthermore, the large observed standard deviation of spacings is in conflict with the relaxation models presented, all of which would predict a narrow distribution of crack spacings. In that discussion, the original distribution of flaws (which must be present to give rise to cracks) was assumed to be unimportant, that is, uniform on a finer scale than the crack spacings and thus able to be ignored.

With this assumption, Atkinson and Guppy considered the elastic relaxation model of Grosskreutz and $\mathrm{McNeil}^{7}$ for intercrack spacing. To understand this model, it should be pointed out that while some of the figures above portrayed clear delineations between strain-released regions and strained regions, in actuality the degree of strain release gradually decreases with distance from a crack (Figure 4.17), so that the driving force for additional cracking increases gradually with distance and is greatest midway between existing cracks.

Based on this consideration, Grosskreutz and McNeil predict the intercrack spacing $d$ to be given by

$$
d \approx \frac{4 h}{\ln \varepsilon / \varepsilon_{o}}\left[1-d / d_{o}\right]
$$

where $h$ is the film thickness, $\varepsilon$ is the final strain which gives rise to spacing $d$, and $\varepsilon_{0}$ is the strain at the time of spacing $d_{o}$ (by strain is meant the shrinkage strain that would occur in an unconstrained film). If the $\left(\varepsilon_{0}, d_{0}\right)$ data point is chosen to be the appearance of the first crack, then $d_{0}$ is infinity and the equation becomes

$$
d \approx \frac{4 h}{\ln \varepsilon / \varepsilon_{o}}
$$

Following Atkinson and Guppy, the final strain can be described in terms of $h_{c}$ using equation 4.3 :

$$
K_{l_{c}}=\int E \varepsilon \sqrt{h_{c}}
$$


While that step is easily justified, Atkinson and Guppy go on to describe $\varepsilon_{\mathrm{o}}$ in terms of $h$ using the same equation, ignoring the necessity of an existing flaw of size $1 / 2 h$ before the first crack can form. The resulting equation gives the spacing a complicated dependence on $\mathrm{h}$ which does not fit the data, and they conclude that the elastic model is inappropriate.

However, it could be argued that the sol-gel process should give rise to flaws with a characteristic size distribution which is independent of film thickness, such that a flaw with size $1 / 2 h$ will not necessarily be present for arbitrary $h$. It should also be kept in mind that the flaws are probably growing during the period of increasing strain. Since a critical-size flaw is needed before any cracking can begin, $\varepsilon_{0}$ cannot be described in terms of $h$, but instead should be described in terms of the largest flaw present, using the appropriate energy balance. That is, we assume that as the strain gradually increases, unstable cracking first becomes favorable $\left(h_{c}<h\right.$ for the given strain) but the first crack still will not form until $h_{c}<2 c$, where $c$ is the size of the largest flaw present. With this assumption, the equation for crack spacing becomes

$$
d \approx \frac{8 h}{\ln 2 c / h_{c}}
$$

If the size of the largest flaw, $c$, is assumed to be independent of film thickness, then the log term is a constant and the spacing, $d$, is given a simple linear dependence on film thickness $h$. Thus, the elastic relaxation model, when modified to have a flawsize dependence, agrees with the observed cracking behavior.

In the present research and in previous studies 4 , the mean spacing $d$ has been found to be approximately ten times the film thickness. For equation 4.7 to fit that data, $c$ should be approximately equal to $h_{c}$. While it is somewhat of a coincidence that, regardless of film thickness, the characteristic flaw size should be 
roughly equal to the critical thickness, this relationship is easily conceivable for films much thicker than $h_{c}$. In films less than twice the critical thickness, on the other hand, flaws of such sizes would be greater than the critical size before the stress state causes the film to be above the critical thickness. In this case, isolated through-thickness cracks would be present before unstable cracking begins; this is precisely the behavior seen by Atkinson and Guppy in films just above the critical thickness ${ }^{4}$. Since these isolated cracks are clearly not unstable, there is no reason to ask them to conform to the elastic model in which spacing is determined by instability according to film thickness. The flaw-modified model, on the other hand, should still be applicable (and agrees with the data).

The large standard deviation is also readily explained using the same model. Since different areas of the sample will have slightly different flaw sizes, cracking will begin at different values of strain. Furthermore, the elastic model assumes that new cracking will occur midway between existing cracks, whereas in reality this midpoint merely represents the most probable location of a crack based on driving force; the actual crack location will also depend on where the largest remaining flaws are located (Figure 4.18). Thus, a variety of crack spacings should arise from the modified elastic relaxation model.

Further evidence of the applicability of elastic model can be found if the shortest crack spacings are examined: In the sol-gel derived films studied here, the region in which the crack driving force is extinguished is quite local around the crack, as evidenced by the ability of new cracks to form very near to existing cracks. Since each crack relieves stress only in the direction perpendicular to the crack, it should be expected that near-right angle intersections between cracks could form easily. On the other hand, a near-parallel crack would be expected to lose driving force as it approached a pre-existing crack. Indeed, such cracks can sometimes be seen to arrest when they come close enough to an existing crack (Figure 4.19). This 
is consistent with the idea of elastic relaxation determining crack spacing, rather than some sort of large-range plastic deformation or delamination.

Furthermore, plastic relaxation or limited decohesion models seem inappropriate for describing cracking with such a large standard deviation of spacing. An example of a sol-gel derived film in which delamination appeared to be important for determining intercrack spacing was seen in an abnormally thick region formed by the meniscus of a coating solution on a silicon substrate (Figure 4.20). Remarkably, the cracks are seen to form parallel to one another in a grid pattern, dividing the film into clearly delaminated rectangular chunks. At this thickness it is clear that the delaminated regions are large enough to interact with neighboring cracks, although the sequencing of such crack formation is quite difficult to explain. What is important here is the stark contrast between this grid pattern of cracking and the typical random network seen in normal films, when the spacing of cracks is determined by defect location and elastic relaxation rather than by the extent of delamination.

\section{Effect of Substrate Porosity on Crack Growth}

In the case of a porous substrate, the first thing which needs to be understood is the reduction in the value of the critical thickness. Stable crack growth appears to be aided somewhat by partial delamination near the crack. The critical film thickness, then, should have some dependence on the ease of film delamination. The reduction in critical thickness from approx. 0.5 microns to 0.2 microns when a $50 \%$ porosity substrate is used (as in figure 7 ) could be explained by invoking the reduction in the energy of delamination. This "delamination" is actually just an averaged reduction in the total area of interface which must be created when the delaminated area bordering the crack is formed. Unstable cracking will be possible 
at lower film thicknesses because the additional surface energy already present at the tops of pores can be used in the energy balance.

The reduction in critical thickness to less than half the previous value seems rather excessive when considering the apparently minor role of delamination in the energy balance for cracking. However, the presence of substrate porosity may enhance delamination to a much greater extent than would be suggested by the mere percentage change in surface area. When a crack develops in the film above a substrate pore, stresses could be expected to concentrate at the pore walls (Figure 4.21), making delamination much easier there than it would be for a corresponding area on a continuous substrate. Thus, the delamination zone associated with each crack is probably much wider in films on porous substrates.

When considering the stable propagation of a crack, the energy balance argument holds only if the pore opening effect is averaged over the path of the crack. Thus, pore walls, which represent areas of complete adhesion, can only be crossed by a propagating crack if the length of pore wall which must be crossed is small compared with the active length of crack (the leading section) in which the strain energy is being released (Figure 4.22). The length of crack over which such strain release occurs is known to be of the order of several film thicknesses 8 ; this means that in films near the critical thickness on 0.2 micron pore size membranes (see Figure 4.12) the pore walls are roughly of the same length as the active length of crack.

The propagation of a critical-size defect, then, becomes a probability question. When the defect forms over a pore, it can grow aided by complete "delamination" until it reaches the first pore wall. The crack may or may not have the ability to cross the pore wall, depending on the local pore geometry and film thickness. The probability of this event is essentially zero at the reduced critical thickness, so that below this thickness all cracks are seen to stop at pore walls ("pore 
induced cracking" behavior is seen). The probability increases with film thickness, so that both types of cracks can be seen at thicknesses just above the reduced critical thickness. When the film thickness approaches the critical value for continuous substrates, the probability of propagation should be unity (all critical size defects propagate) and hence above this thickness only long, unstable cracks can be seen, as in Figures 4.7 and 4.8. The thickness dependence of cracking behavior is outlined in Figure 4.23.

Without considering such a probability dependence, it would be quite difficult to explain the region of Figure 4.12 where both types of cracking are operative; the presence of any unstable cracks would incicate that all of the visible defects should have been unstable. (A possible explanation which considers relaxation near existing cracks fails to explain the random geometrical nature of the crack occurrence.)

\section{Mechanism of Pore-Induced Cracking}

Earlier in this discussion, it was argued that the sol-gel process should give rise to a characteristic distribution of defects during consolidation. In the case of very thin films on porous substrates, the frequency of pore-induced cracking indicates that the concentration of defects has been increased. More specifically, the frequency of this type of cracking is seen to increase with increasing pore size and decreasing film thickness. Possible mechanisms for such behavior will be discussed next, keeping in mind that whatever mechanism is put forward, the expected behavior must tend toward what appears to be a completely uncoated substrate as zero film thickness is approached.

One possible explanation considers the stress concentrations in the film that might arise at pore walls during processing. Each pore could be expected to give rise to film thickness variations, and stress concentrations due to the changes in 
thickness would increase with increasing pore size and decreasing film thickness. The severity of such a defect would be greatest when the film thickness is of the same order as the pore size, or less. Some possible models for pore-induced stress concentrations are presented in Figure 4.24. These models, of course, depend on the film profile that is left behind by the solvent evaporation within the pores.

Pore-induced cracking could also be expected if the film is still in a gel or liquid state when the opening forms ${ }^{9}$ : When the pore begins to empty during solvent evaporation and the triple interface reaches the top of the pore, the wetting angle might be such that the thickness of the film is reduced over the top of the pore (Figure 4.25). When the film thickness is roughly the same as the pore diameter, an opening would be expected to form over the pore. This scenario is in agreement with the observed dependence of pore-induced cracking on film thickness; poreinduced cracking is seen to begin when the film thickness is roughly the same as the pore size.

When film thicknesses are smaller than pore diameters, the pore-induced discontinuities in the film lose their crack-like character; they begin to appear as if they formed during the liquid stage. It may be useful, then, to consider the favorability of film continuity through the simple criterion of the total surface energy of the liquid. If a circular opening forms above a pore, the "crack" surface area added is equal to $2 \pi \mathrm{rh}$, whereas the missing disc of film had top and bottom surfaces with total area $2 \pi r^{2}$ (Figure 4.26). Equating these two surface areas, we find that the total energy of the film will be reduced when $r>h$. Thus, the minimization of surface energy represents a criterion which explains the film behavior perfectly, although it is difficult to propose a mechanism by which this criterion could always be followed. 
While these arguments could help to explain the dependence of poreinduced cracking on substrate pore size and film thickness, further research clearly must be done before their accuracy can be assessed.

\section{E: Summary of Mechanical Behavior Results}

'Two different types of cracking were observed in the sol-gel films, each occurring for a well-defined film thickness regime. Long, unstable shrinkage cracks occurred at film thicknesses greater than a critical value, while localized cracks associated with substrate porosity (pore-induced cracking) were found only in films thinner than a critical value. Crack-free films were formed when these two cracking rnechanisms did not have overlapping thickness regimes; this condition was met when the size of the substrate pores is below a critical value.

For yttria-stabilized zirconia films produced from ispropoxide solutions, films must be thinner than about 0.5 microns on continuous substrates, or 0.2 microns on $50 \%$ porosity substrates, before the long unstable cracks are eliminated. In order for the localized, pore-induced cracking to be limited to films thinner than 0.2 microns, the size of the pores must be limited to about 0.02 microns. Films and substrates for fuel cell applications or other electrolyte devices apparently must conform to these limitations before crack-free sol-gel derived membranes can be formed.

It has also been shown above that the occurrence and distribution of the unstable cracks can be explained by a modified elastic relaxation model when simple assumptions about the flaw distribution are made. This result should be applicable to any film coating process which gives rise to a significant shrinkage and a population of defects.

Film behavior peculiar to porous substrates has also been discussed: The reducrion in critical thickness when a pornus suhstrate is used can he explained by 
invoking the additional surface energy available above the pores, and considering crack propagation from a probability perspective. Some models which account for pore-induced cracking have also been proposed above, but further experimentation would be needed to test these ideas.

Film delamination was also seen for thick films on brittle, porous substrates, and the delamination crack was observed to dive into the substrate; while this phenomenon is unimportant for electrolyte applications (it was only seen in thick films), it is interesting to note that the behavior confirms the predictions of $\mathrm{Hu}$ and Evans in which a stable delamination crack should follow the plane defined by $\mathrm{K}_{\mathrm{II}}$ $=0$.

\section{References--Chapter 4}

1 J. Livage, "Synthesis, Structure, and Applications of $\mathrm{TiO}_{2}$ Gels," pp. 717-724 in Materials Res. Symp. Proc. Vol. 73, ed. C. J. Brinker, D. E. Clark, and D. R. Ulrich, Mat. Res. Soc., 1986.

2 S. Sakka, K. Kamiya, K. Makita, and Y. Yamamoto, "Formation of Sheets and Coating Films From Alkoxide Solutions," J. Non-Cryst. Solids, 63 223-235 (1984).

3 I. Strawbridge and P. F. James, "The Factors Affecting the Thickness of Sol-Gel Derived Silica Coatings Prepared by Dipping," J. Non-Cryst. Solids, 86 381-393 (1986).

4 A. Atkinson and R. M. Guppy, "Mechanical Stability of Sol-Gel Films," J. Mat. Sci 26 3869-3873 (1991).

5 M. Hu, M. Thouless, and A. Evans, "The Decohesion of Thin Films From Brittle Substrates", Acta Metall. 36 [5] 1301-07, (1988).

6 K. Miller, F. Lange, and D. Marshall, "The Instability of Polycrystalline Thin Films: Experiment and Theory", J. Mater. Res. 5 [1] 151-160 (1990). 
7 J. C. Grosskreutz and M. B. McNeil, "The Fracture of Surface Coatings on a Strained Substrate", J. Appl. Phys. 40 355, (1969).

$8 \mathrm{~J}$. W. Hutchinson and Z. Suo, "Mixed Mode Cracking in Layered Materials", Adv. Appl Mech. 29 64-187 (1991).

${ }^{9}$ R. Gronsky, private communication. 


\section{Chapter 5: Suitability of the Process: Electrolyte Device Considerations}

This section is mainly a discussion of the implications of the results obtained in earlier chapters. Some additional results are presented concerning macroscopic testinis of large-area films. Additional processing steps which would be necessary for various electrolyte applications are also discussed.

\section{A: Quality of the Electrolyte Film}

While it was determined in Chapter 4 that crack-free films could be formed on porous substrates, it was unclear how difficult it would be 10 control the process to obtain continuous films over large areas. Consequently, some large-area tests were performed to assess the actual continuity of these films.

\section{Vacuum Leak Testing}

A helium/vacuum leak testing apparatus was used to see if gas-tight films could be made over an approximately $1 \mathrm{~cm}^{2}$ area. Coated 0.02 micron porosity Anotec membranes with a film thickness in the experimentally determined "crackfree" region (see Chapter 4) were placed on a copper mount which had holes drilled in it to serve as a rigid porous support. The membrane was then sealed to the copper around the edges using high-vacuum epoxy (Figure 5.1). The copper mount was attached to a vacuum line; the degree of vacuum which could be pulled on the membrane served as an initial test of gas-tightness. An uncoated Anotec membrane was also tested to serve as a control for the apparatus.

It was found that, while the uncoated membrane would only allow a vacuum of 635 torr to be maintained, the same pumping system was able to pull a full (760 torr on the gauge) vacuum on the coated membrane.

The more sensitive helium leak test was also performed: In this test, a helium jet is aimed at the areas where leaks are expected, and a mass spectrometer 
in the vacuum line determines the amount of helium which passes through the leak. Unfortunately, the leak rates of the coated membranes tested were off-scale for the high-sensitivity instrumentation used; apparently the "crack-free" films have a significant amount of discontinuity which allows gas flow to occur. Such leakage represents a chemical "short" for electrolyte devices which will reduce effeciency; film continuity must be improved in order to limit losses to a tolerable level.

\section{Electrode Separation Behavior}

Sol-gel films were deposited on metallic or metal-coated substrates in order to study electrical behavior. The substrate served as one electrode for resistance measurements, while a sputtered or painted-on metal coating over the sol-gel coating served as a second electrode. Simple direct-current resistance measurement revealed a short between these two electrodes for each configuration tested; both a sputtered gold layer and a baked silver paint coating apparently were not prevented from making contact with the substrate through the sol-gel film. While the shorting of the baked silver paint may be due to film damage from thermal stresses, the failure of the sputtered electrodes indicates that when large areas of the film are used, there will always be some defect regions which cause the film to be discontinuous.

\section{Quality Control}

The most common film defect areas observed in microscopy appeared to result from either dust particles (which caused local cracking) or from spot-like regions of a different film thickness. Both of these types of defects are probably due to contamination of the coating solutions or the substrates.

Dust particles which cause local cracking indicate that clean-room processing is necessary both during solution preparation and during the deposition step in 
order to produce large-area defect-free films. While such facilities were not readily available for this research, clean room experimentation should be done to determine the feasibility of producing truly crack-free films.

The spot-like regions of different film thickness were present in films on both continuous and porous substrates. They ranged from very small areas to about $\mathbf{5 0}$ micron diameter spots, within which the film was either thinner than the surrounding coating, or in some cases appeared to be completely absent. These defects possibly arose from inhomogeneous coating solutions; small droplets of an impurity second phase in the liquid or gas bubbles could explain their appearance. The elimination of such defects would probably require more careful preparation of the solutions in addition to a clean, controlled environment.

In the absence of such rigorous quality control, useful films could still be obtained if a treatment to fill in the discontinuities were developed. As discussed in Chapter 4, multiple coatings do not appear to be the answer for the production of continuous films, since existing cracks tend to nucleate cracks in subsequent layers. However, the use of an inert filler material to selectively coat the discontinuous areas may represent a more useful strategy. A blocking material would of course render the film useless if it covered large areas of the film, but if such a material were selectively applied only to areas where the substrate were exposed, a useful device could be obtained. A process such as electrophoretic coating may lend itself to the selective coverage of a metallic substrate, since charge would build up only in the places where the metal is exposed.

\section{B: Electrode Formation: Alumina Membrane Substrates}

The 0.02 micron porosity Anotec alumina membranes were successful as porous substrates for crack-free film deposition, but are not ideal as actual fuel cell components: As described in Chapter 1, most device applications would require a 
catalytic electrode to be added to the surface before electrolyte deposition. In addition, the alumina substrate presents a problem because it represents an unwanted electronic insulator in a current path. In a fuel cell stack, for example, the current must flow from the bottom surface of one electrolyte film to the top surface of the next film. A conductive bipolar plate would serve to separate each cell in the stack, and would fuction as a gas separator as well as an electronic conductor (Figure 5.2). In the case of alumina substrates, a path for electronic current between the electrolyte film and the bipolar plate must somehow be provided.

One possible design makes use of the porous electrode/catalytic layer to provide a path for current to the sides of the membrane, where it can be routed around the alumina substrate. The disadvantage of this design lies in the thickness of the electrode layer which would be required for adequate lateral conductivity; in a $4^{n}$ diameter fuel cell stack, a significant lateral current flow would be present. The thickness of the necessary conductive layer would add to the cost, weight, and size of the device.

An alternative design consists of providing a current path directly through the porosity of the alumina membrane. Unfortunately, the porosity is so fine that any attempt to coat the pore walls with metal, through an electroless plating process for example, would block a significant volume of porosity, and would require even more material than the lateral current design. Thus, a strategy involving selective filling of the porosity must be considered.

Since the porous electrode is still available to provide some lateral conductivity, current paths through the substrate could probably be spaced apart to some degree without affecting device performance. Selectively filling in small regions of the substrate porosity with metal may be an attractive compromise between the two designs discussed above. There remains the difficulty of finding a process to form a continuous conductive material within the narrow columnar 
porosity of the alumina membranes. It may be necessary to form larger-diameter holes within the substrate before the feat can be accomplished. Such a step would, of course, provide additional complications, such as the difficulty of forming a continuous sol-gel film over the altered substrate.

\section{C: Use of Graded Porosity Substrates}

The difficulties discussed above make electronically conductive substrates more attractive when considering fuel cell fabrication; a conductive substrate would eliminate the need for an additional current path between cells in a stack. Possible material choices for such a substrate include conductive ceramics such as LSM, porous metals, or cermets such as nickel-zirconia. Properties such as electronic conductivity, thermal expansion, and catalytic behavior could be optimized with the proper material choice. The main obstacle to the fabrication of a stack with this design is the necessity of a fine-porosity $(\approx 0.02$ micron) uniform substrate in order to obtain crack-free films. In order to insure adequate gas flow, the size of the porosity in the bulk of the substrate must be larger than the fine surface porosity, so that a graded-porosity design is required.

Such structures can be fabricated by coating a coarse-porosity material with a fine, uniform powder. However, the tortuosity of any material formed from equiaxed powders will make gas flow rates significantly lower than those for an columnar-porosity membrane of the same pore size. One technique which might minimize the thickness of material which has unfavorable porosity would be use the bipolar plate itself as the base for a graded-porosity structure; if the plate had fine channels in it, then mechanical support would be well-distributed and a relatively thin layer of small-porosity material could be used (Figure 5.3). 


\section{D: Conclusion}

This research has demonstrated that continuous sol-gel derived films can be deposited on porous substrates by a simple spin-coating process in which the substrate porosity is allowed to fill with coating solution. By noting the restrictive conditions under which this procedure is possible, several conclusions about process requirements can be made.

The porous substrate must be thin and without large porosity so that excessive coating solution is not absorbed and so that sol-gel derived material does not form within the substrate.

If cracking in the films is to be avoided, then i) There is a maximum singlestep film thickness of about 0.2 microns, above which unstable cracking will occur; thicker films would require multiple coatings. ii) Porosity at the surface of the substrate must be smaller than the film thickness to prevent pore-induced cracking. iii) Clean-room conditions are needed to insure homogeneous coating solutions and to prevent defect-induced cracking.

Electrolyte devices must be delicately engineered to avoid potential film damage; reactive substrates may destroy film integrity, and electrode attachment or sealing procedures must be performed without applying stress to the film. These considerations make it important to keep device design as simple as possible, and more specifically make it desirable for the substrate to have electronic conductivity.

This requirement, when combined with the need for very fine, controlled porosity at the surface, make substrate fabrication a challenging subject for future research. 


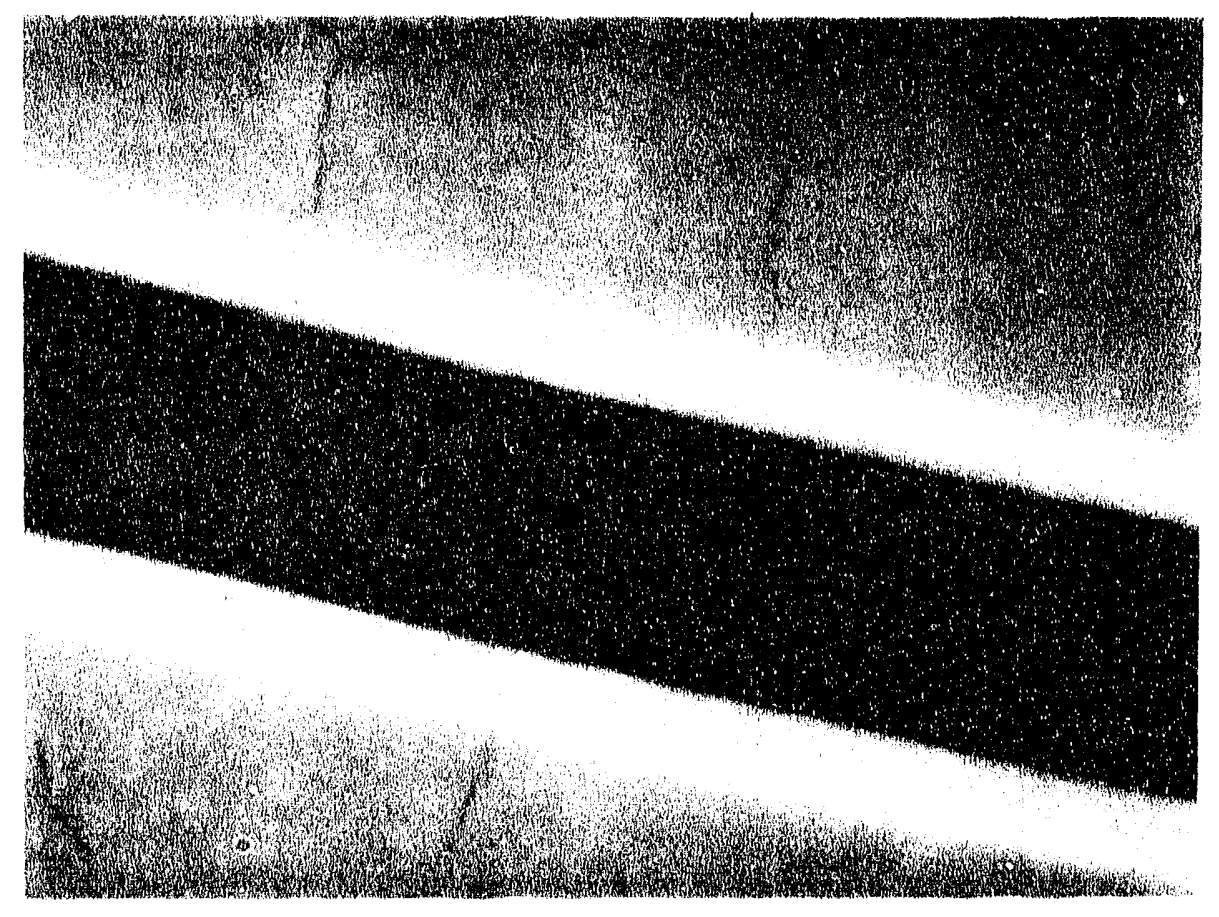

$330 \mathrm{~nm}$

Figure 2.1 SEM micrograph centered on a large crack in a zirconia film on quartz. Ziconia islands can be seen above and below the crack, with smaller cracks nucleating at the large crack. 


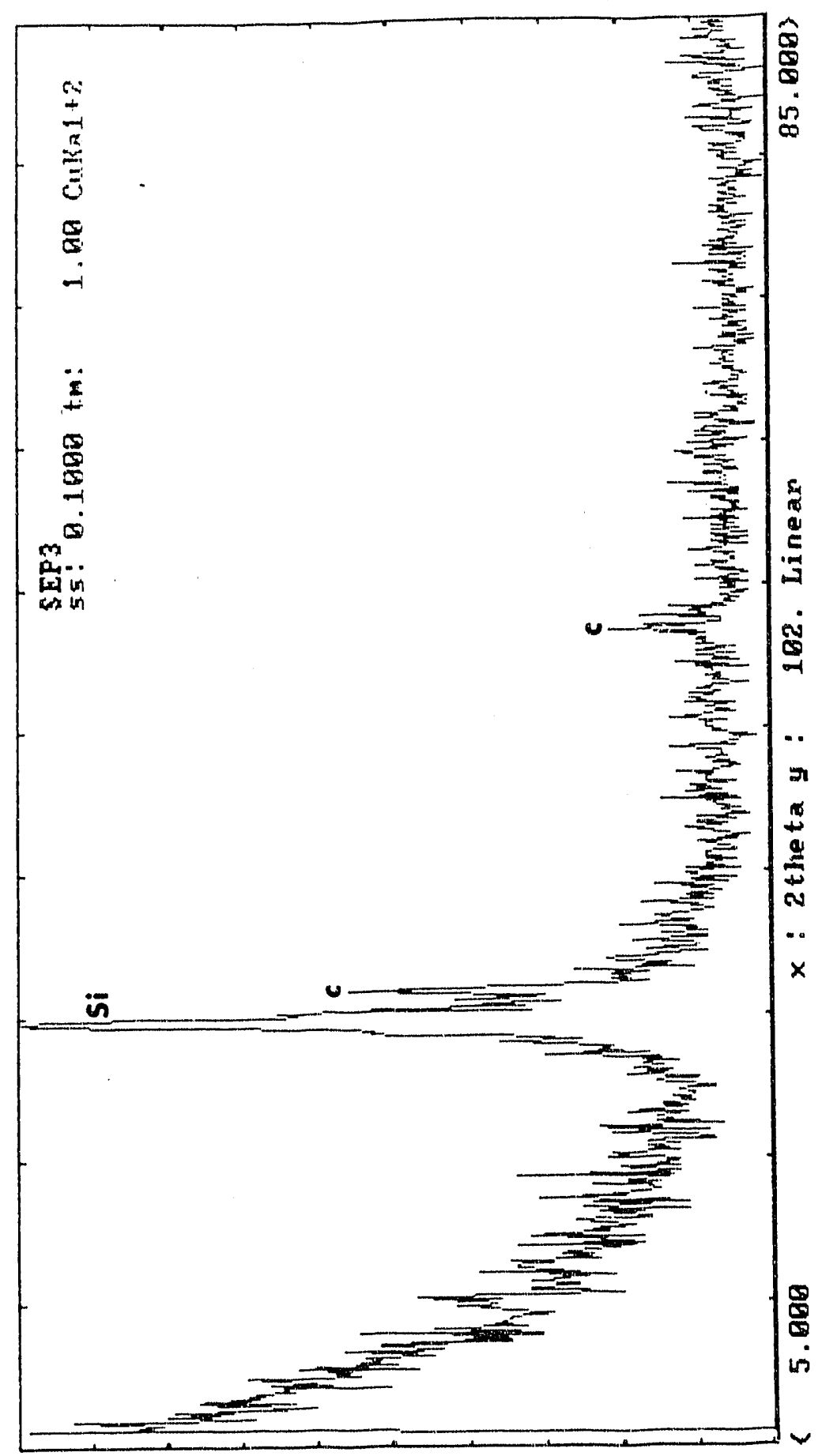

Figure 2.2 X-ray diffraction pattern for undoped zirconium isopropoxide film after $400 \mathrm{C}$ treatment. $\mathrm{c}=$ cubic zirconia, $\mathrm{Si}=(111)$ silicon substrate. 


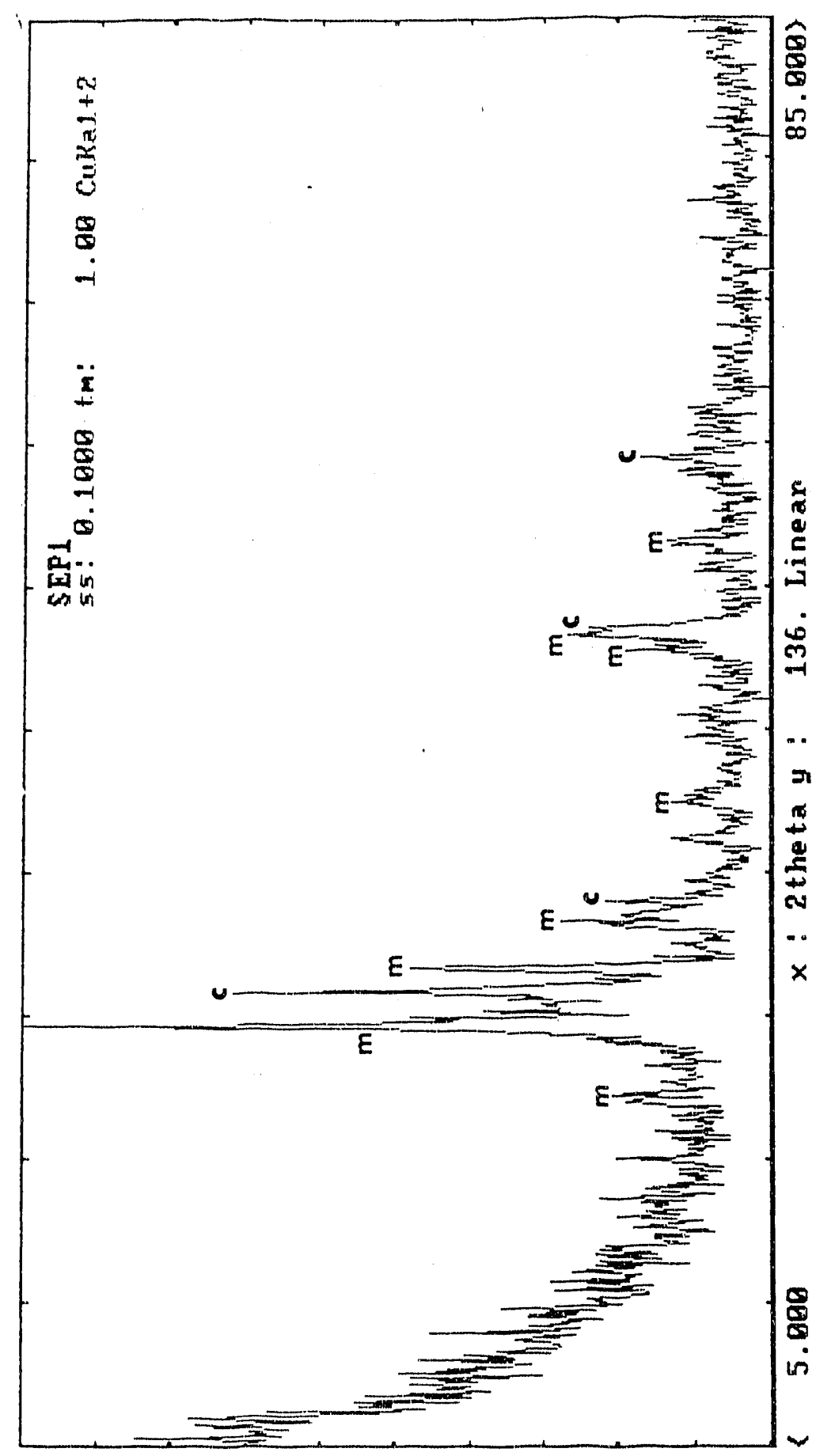

Figure 2.3 X-ray diffraction pattern for undoped zirconium isopropoxide film after $600 \mathrm{C}$ treatment. $\mathrm{c}=$ cubic zirconia, $\mathrm{m}=$ monoclinic zirconia. 


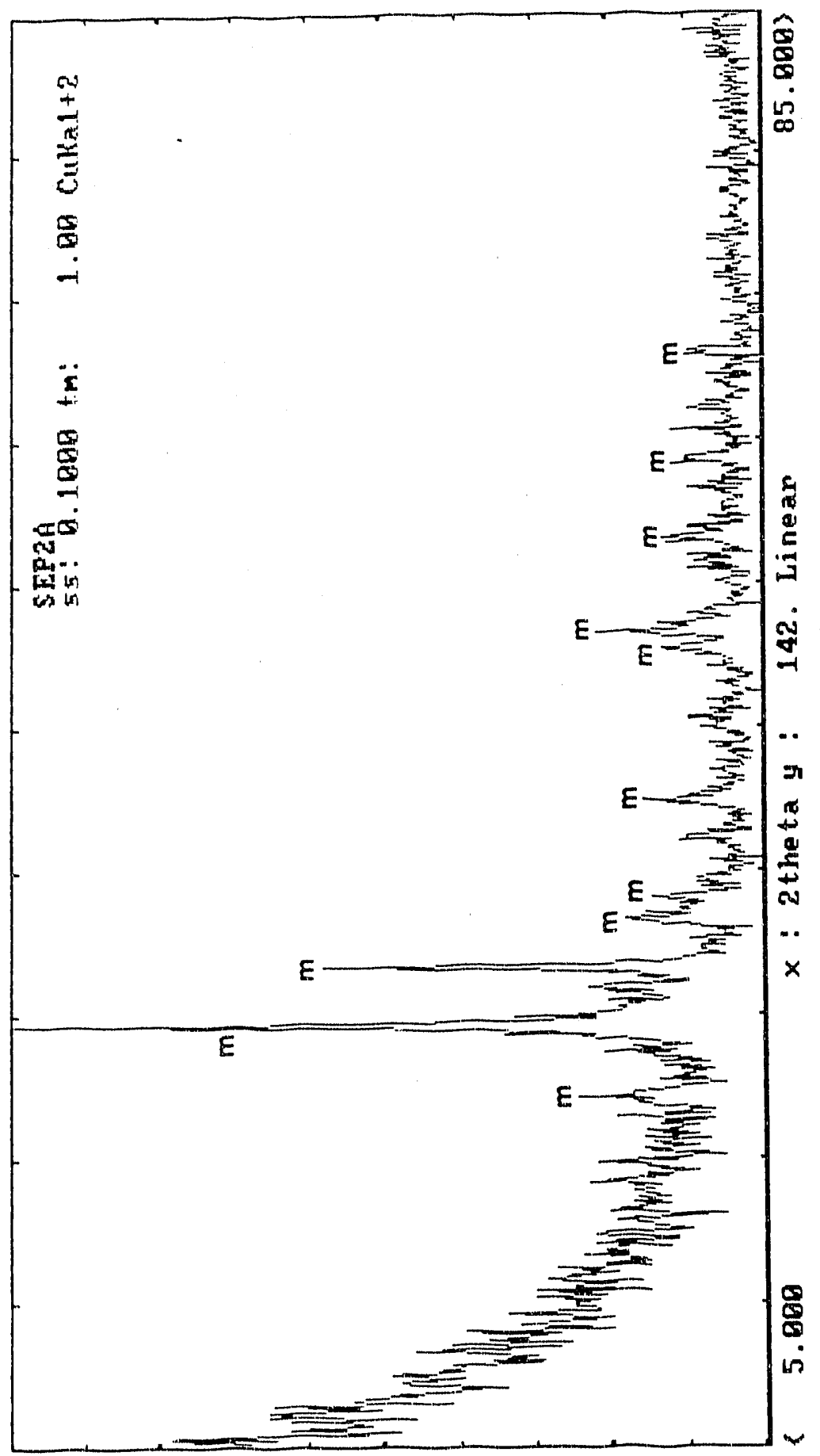

Figure 2.4 X-ray diffraction pattern for undoped zirconium isopropoxide film after $1000 \mathrm{C}$ treatment. $\mathrm{m}=$ monoclinic zirconia. 


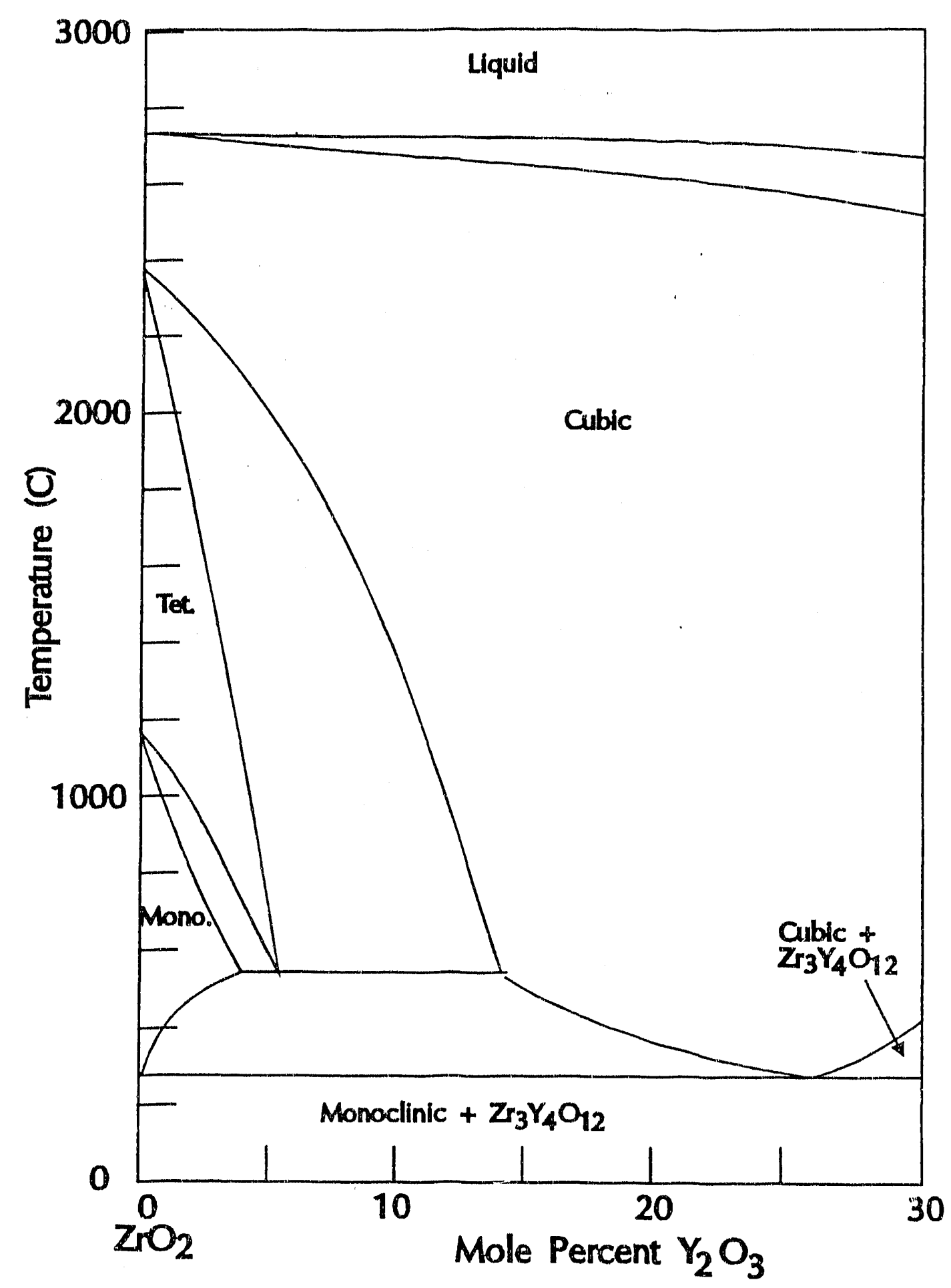

Figure 2.5 Part of the zirconia-yttria phase diagram. 


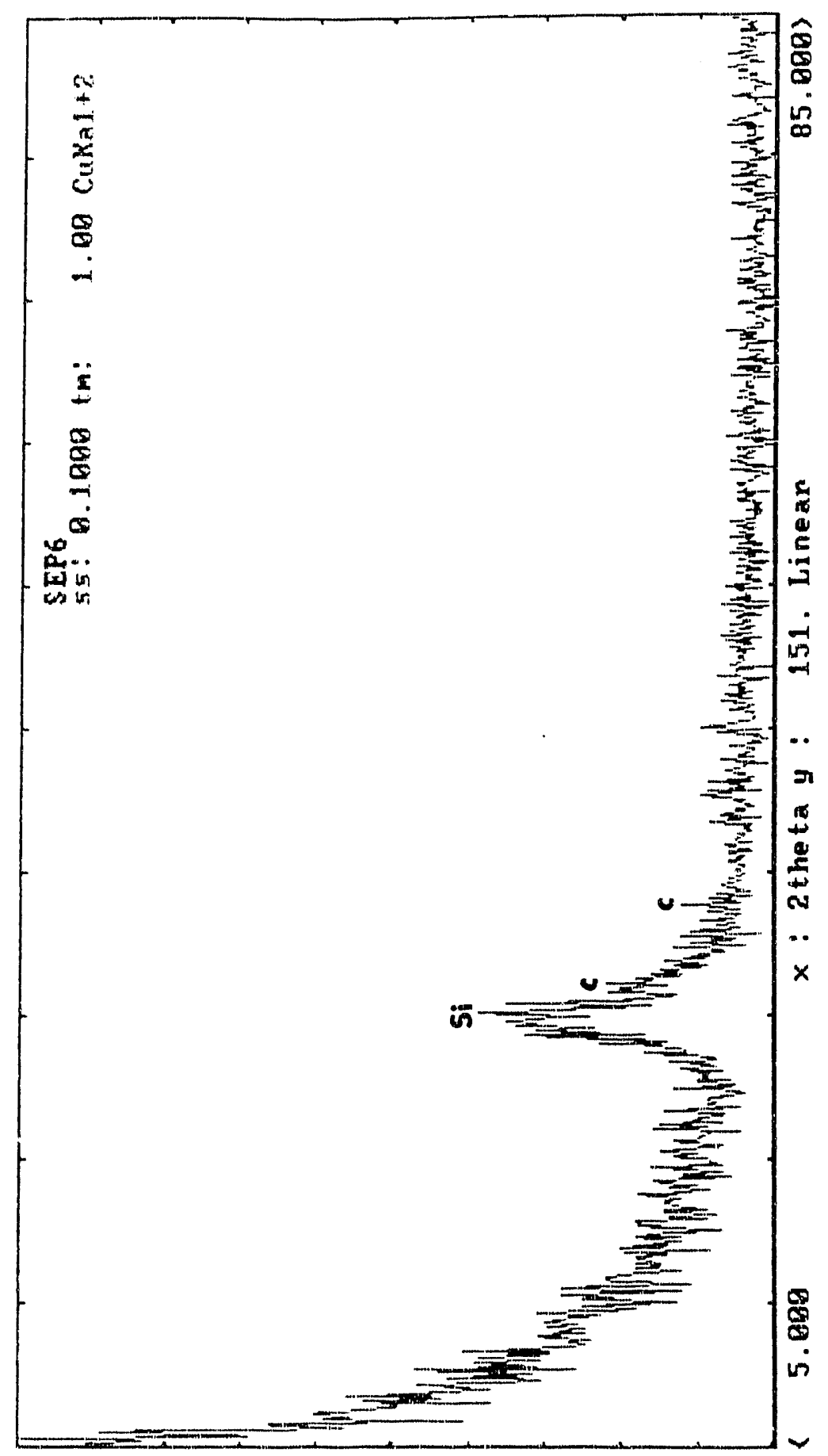

Figure 2.6 $\mathrm{X}$-ray diffraction pattern for a zirconium isopropoxide film doped with 18 mole \% YO $\mathrm{YO}_{1.5}$, after $400 \mathrm{C}$ treatment. $\mathrm{c}=$ cubic zirconia. 


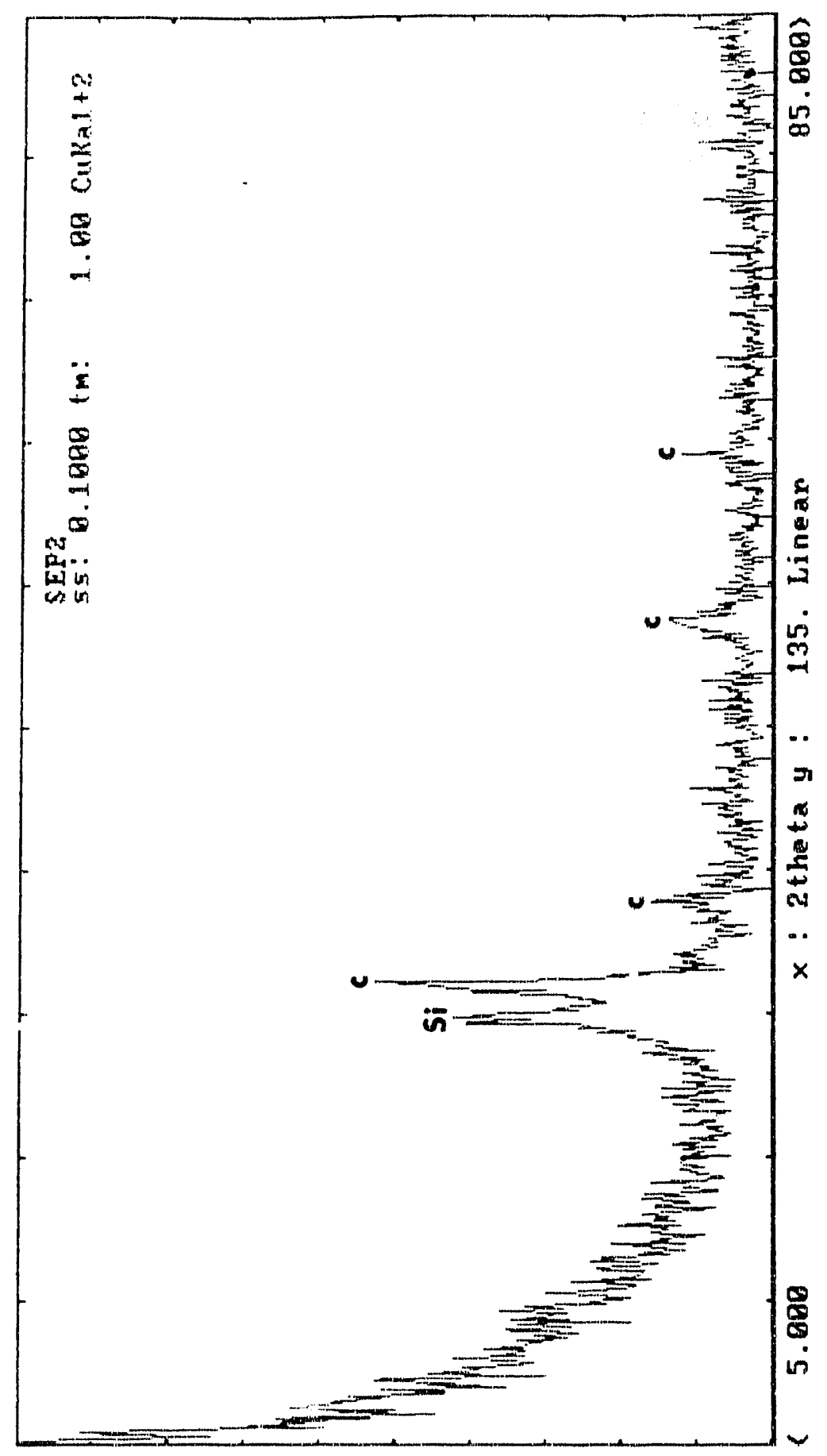

Figure 2.7 X-ray diffraction pattern for a zirconium isopropoxide film doped with 18 mole \% YO 1.5 , after $600 \mathrm{C}$ treatment. $\mathrm{c}=$ cubic zirconia. 


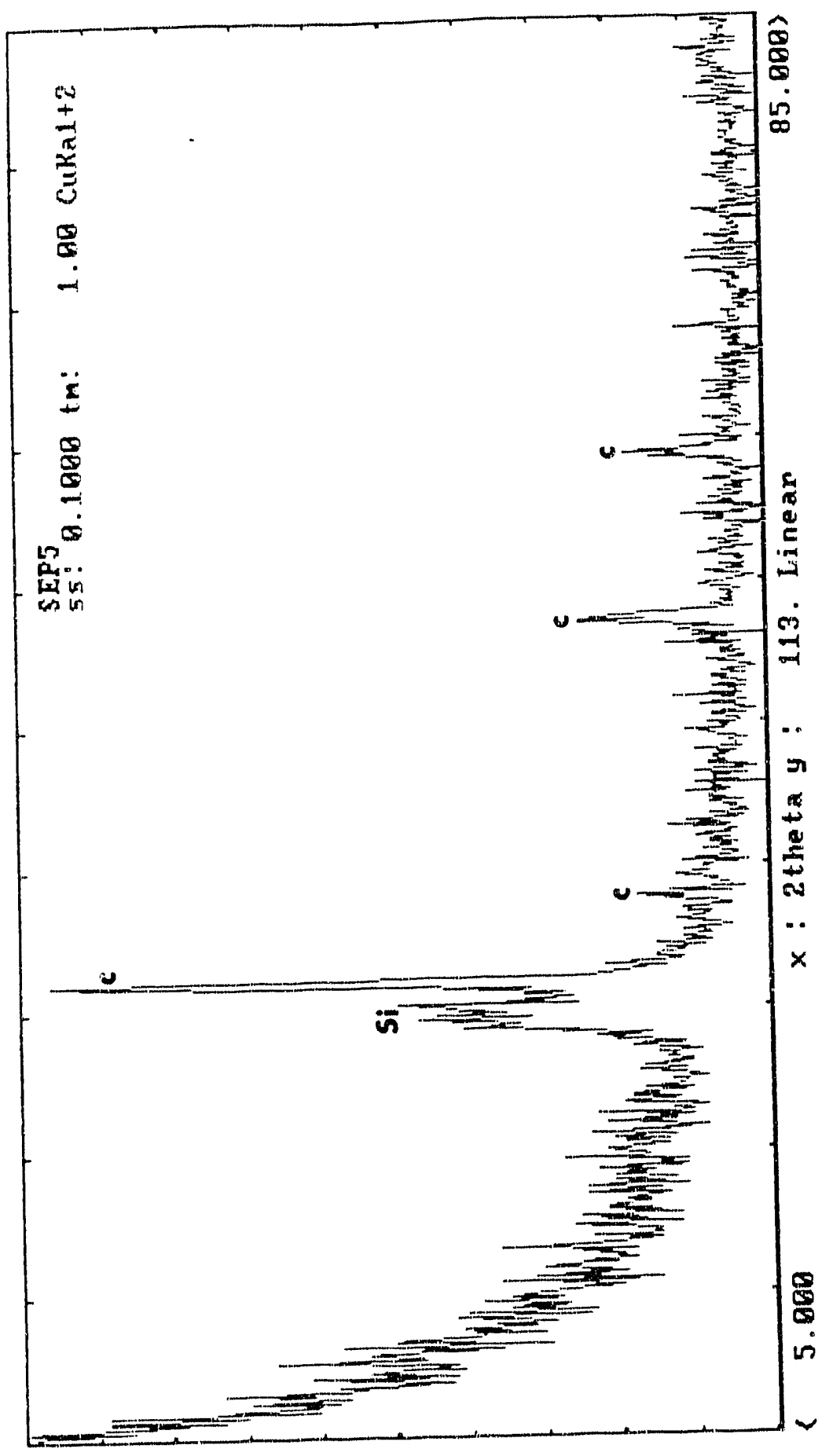

Figure 2.8 X-ray diffraction pattern for a zirconium isopropoxide film doped with 18 mole \% YO 1.5 , after $1000 \mathrm{C}$ treatment. $\mathrm{c}=$ cubic zirconia. 


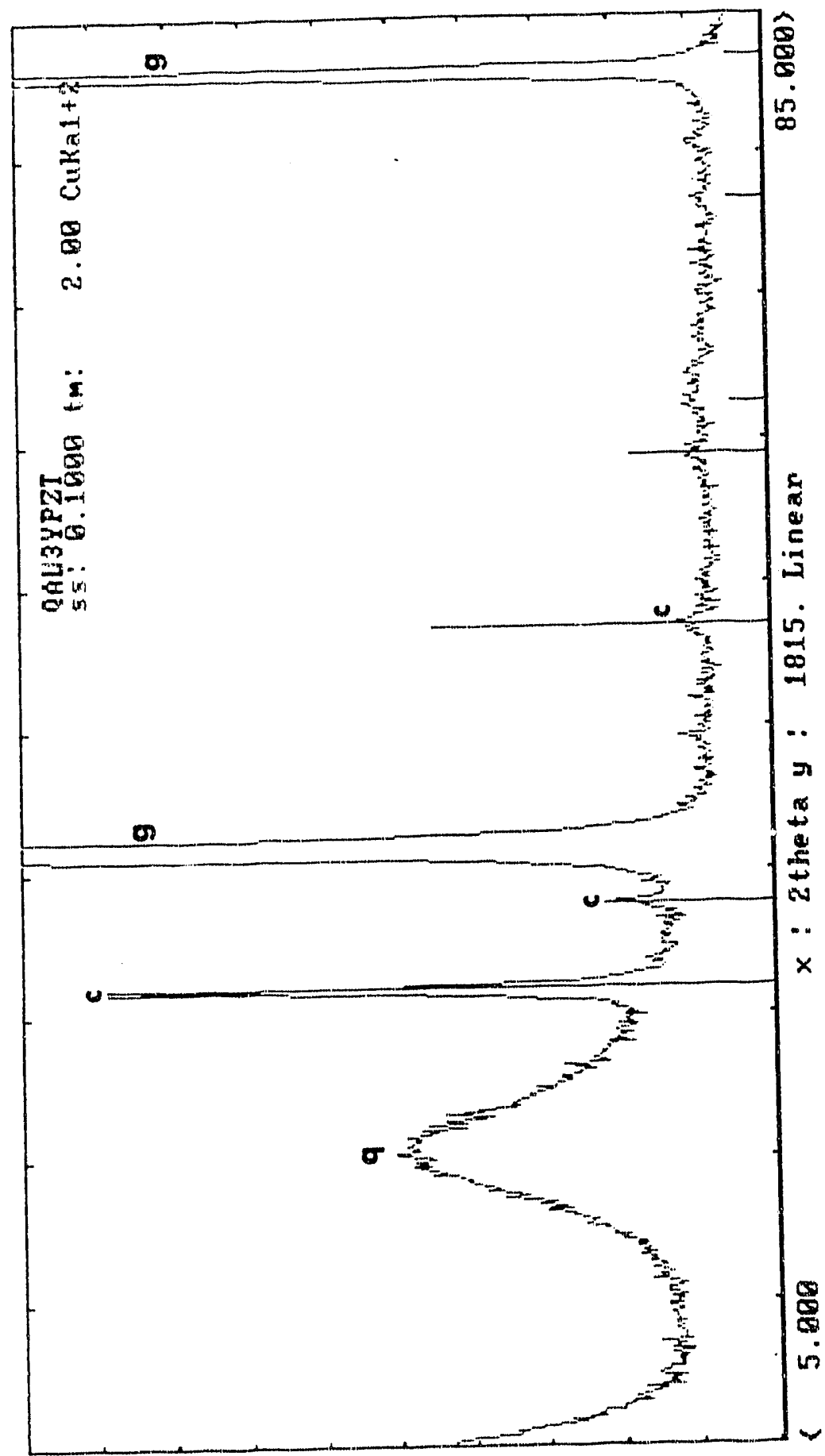

Figure 2.9 X-ray diffraction pattern for a zirconium isopropoxide film doped with 18 mole $\% \mathrm{YO}_{1.5}$, after a $600 \mathrm{C}$ treatment on gold-coated quartz. $\mathrm{c}=$ cubic zirconia, $\mathrm{g}=$ gold, $\mathrm{q}=$ quartz. 


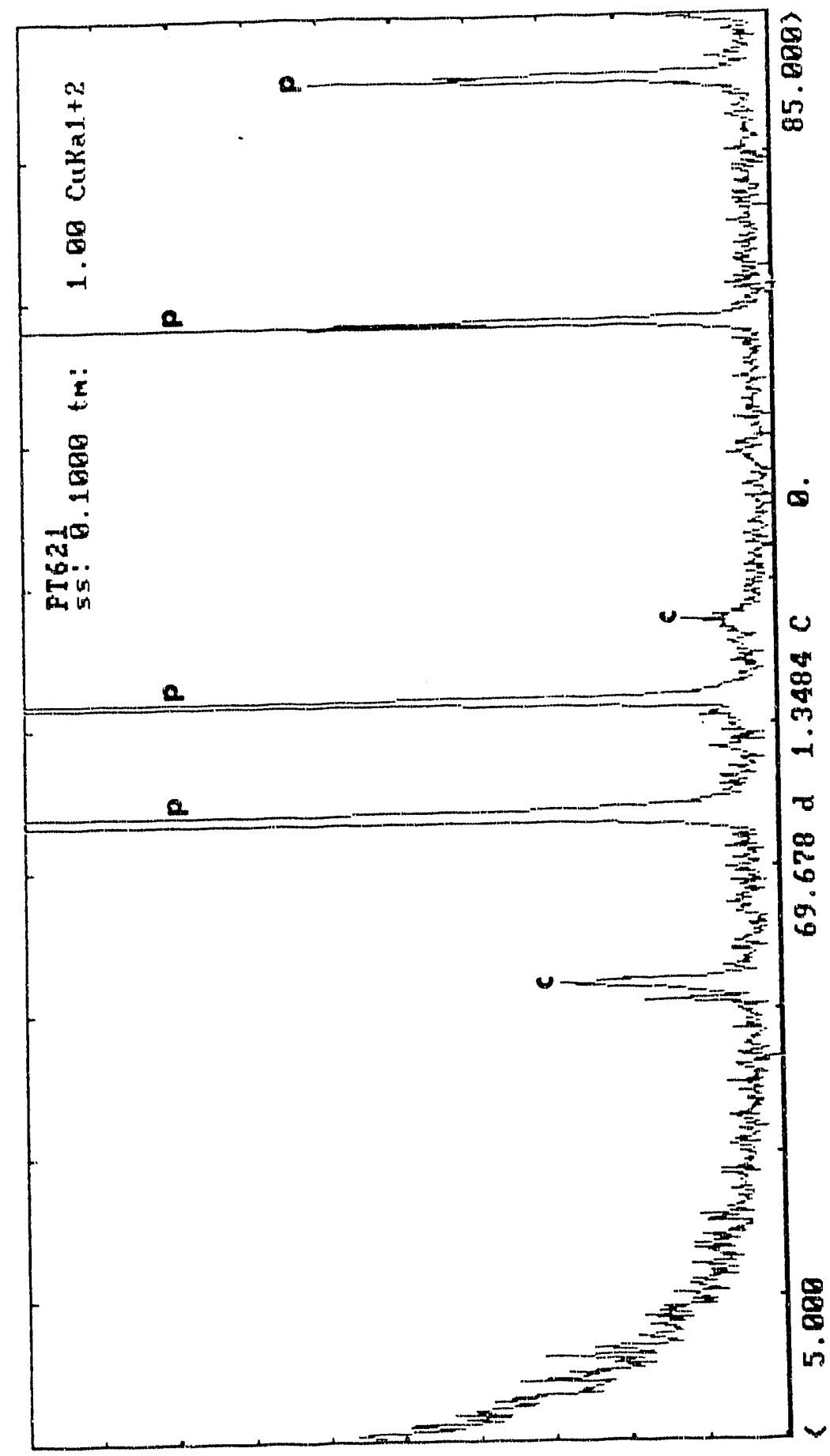

Figure 2.10 $\mathrm{X}$-ray diffraction pattern for a zirconium isopropoxide film doped with 18 mole $\% \mathrm{YO}_{1.5}$, after a $600 \mathrm{C}$ treatment on platinum sheet. $\mathrm{c}=$ cubic zirconia, $\mathrm{p}$ = platinum. 


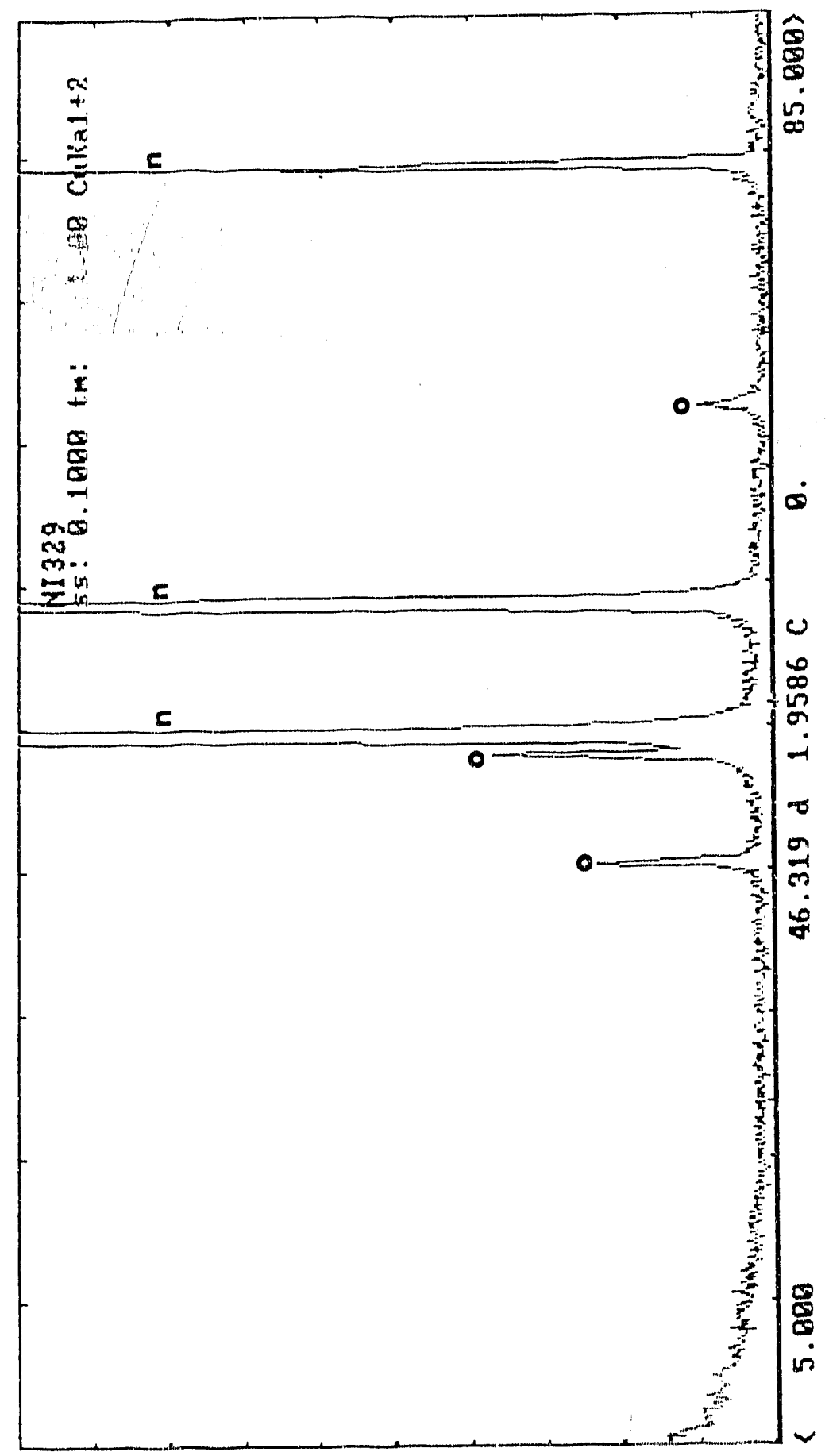

Figure 2.11 $X$-ray diffraction pattern for a zirconium isopropoxide film doped with 18 mole $\% \mathrm{YO}_{1.5}$, after a $600 \mathrm{C}$ treatment on nickel. $\mathrm{n}=$ nickel, o $=$ nickel oxide. 


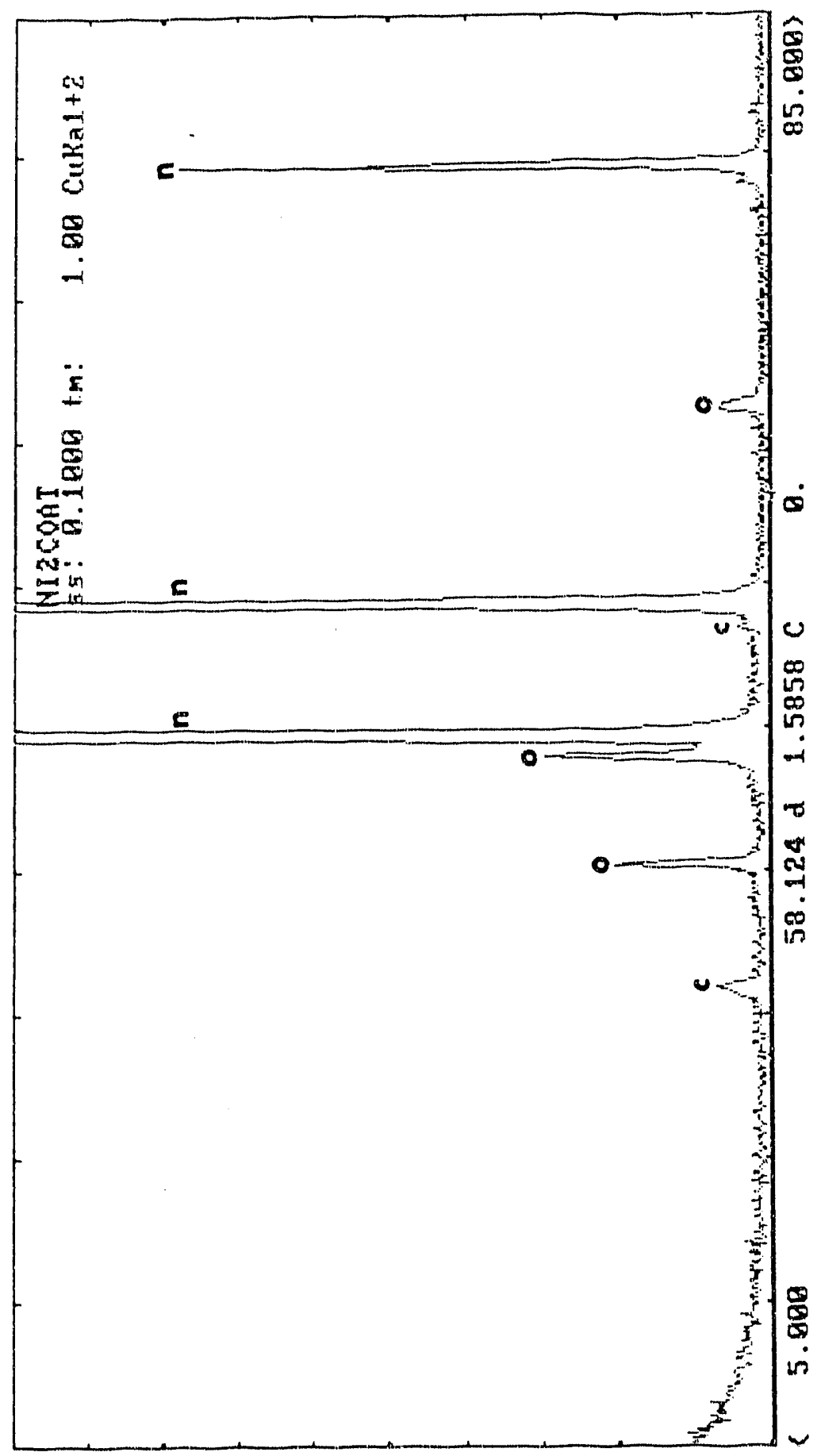

Figure 2.12 $\mathrm{X}$-ray diffraction pattern for a zirconium isopropoxide film doped with 18 mole \% YO 1.5 , after a $600 \mathrm{C}$ treatment on nickel. $\mathrm{c}=$ cubic zirconia, $\mathrm{n}=$ nickel, $\mathrm{o}=$ nickel oxide. 


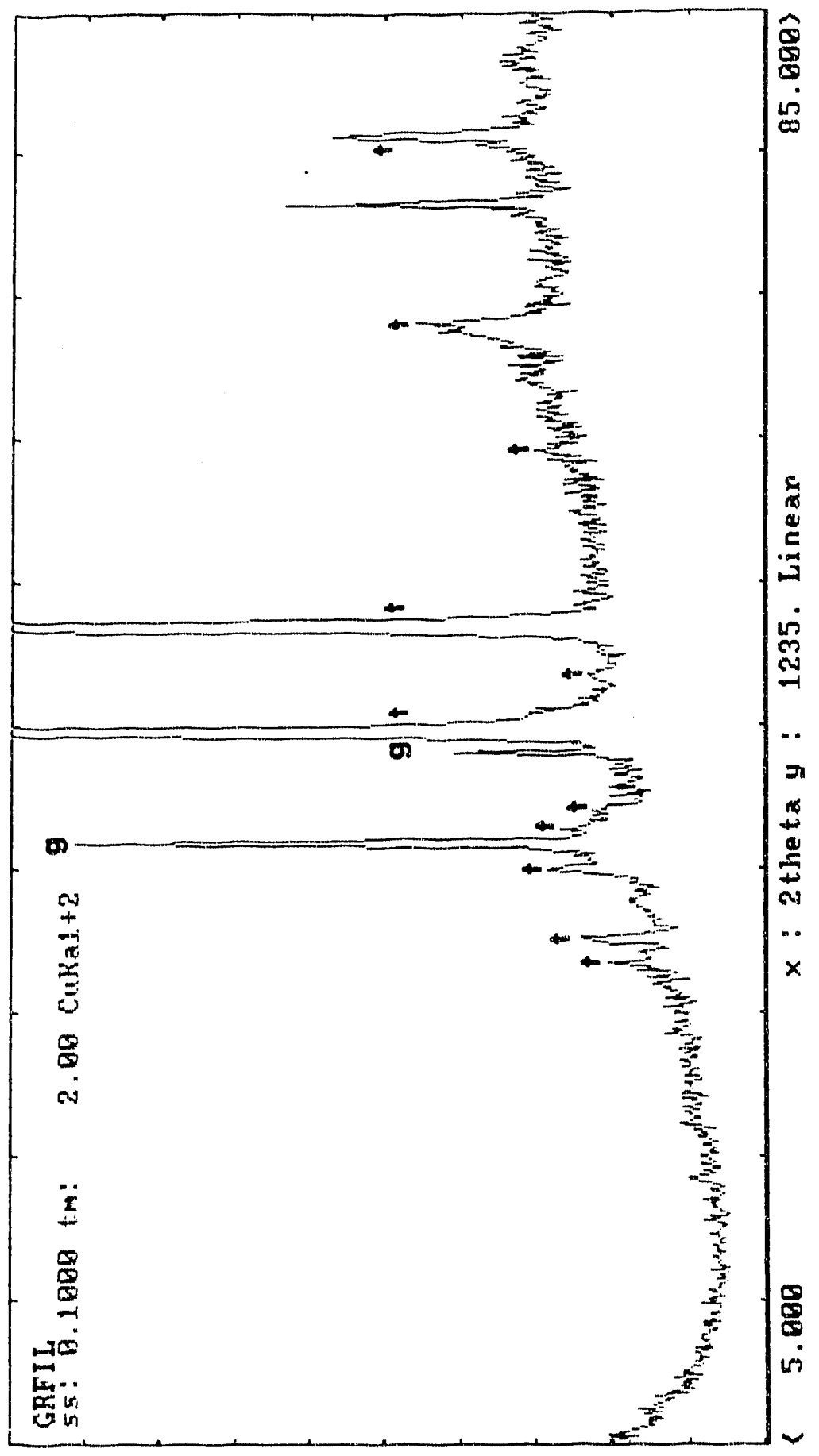

Figure 2.13 $\mathrm{X}$-ray diffraction pattern for an as-supplied Anotec membrane, coated with gold. $\mathrm{t}=\theta$-alumina, $\mathrm{g}=$ gold . 


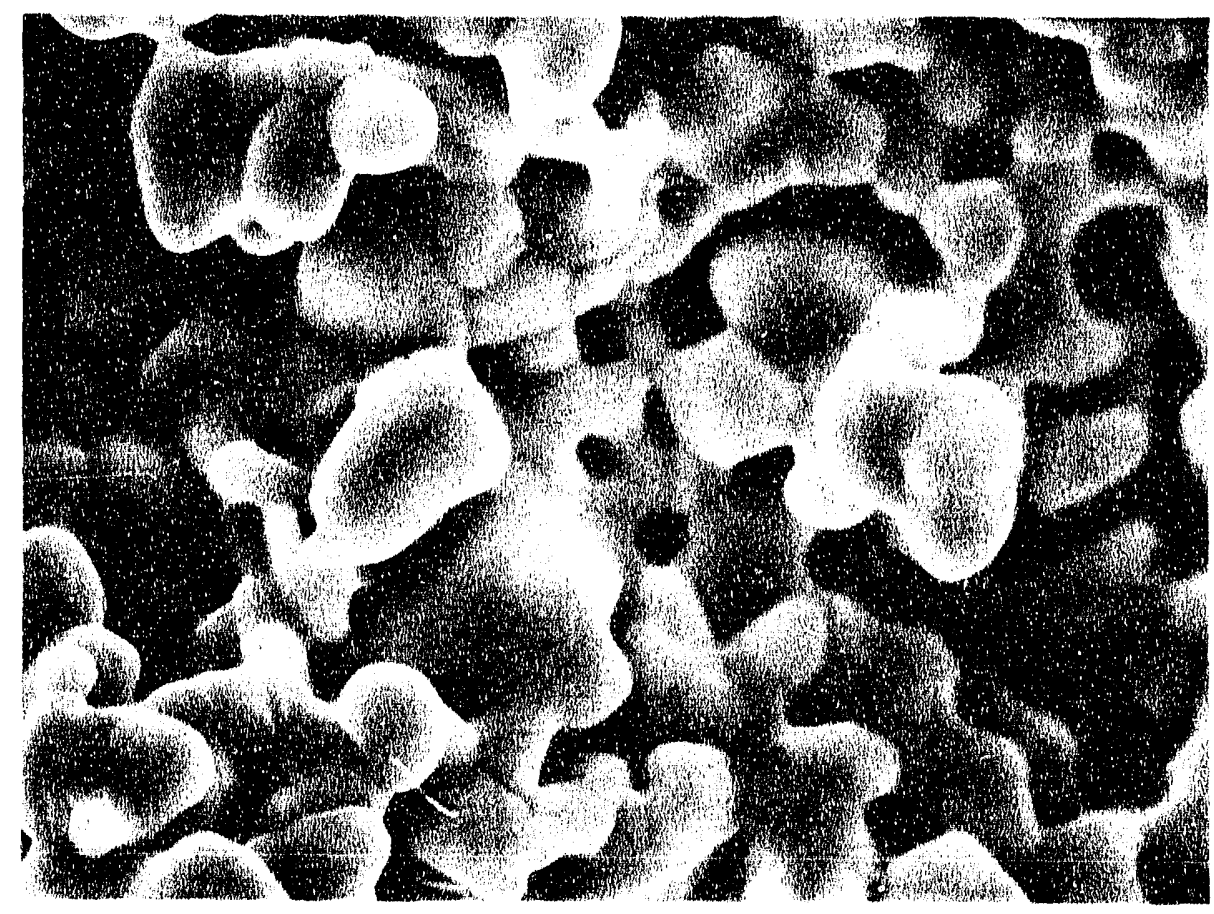

$2.5 \mu \mathrm{m}$

Figure 3.1 SEM micrograph of tape-cast and sintered LSM, showing large surface pores. 


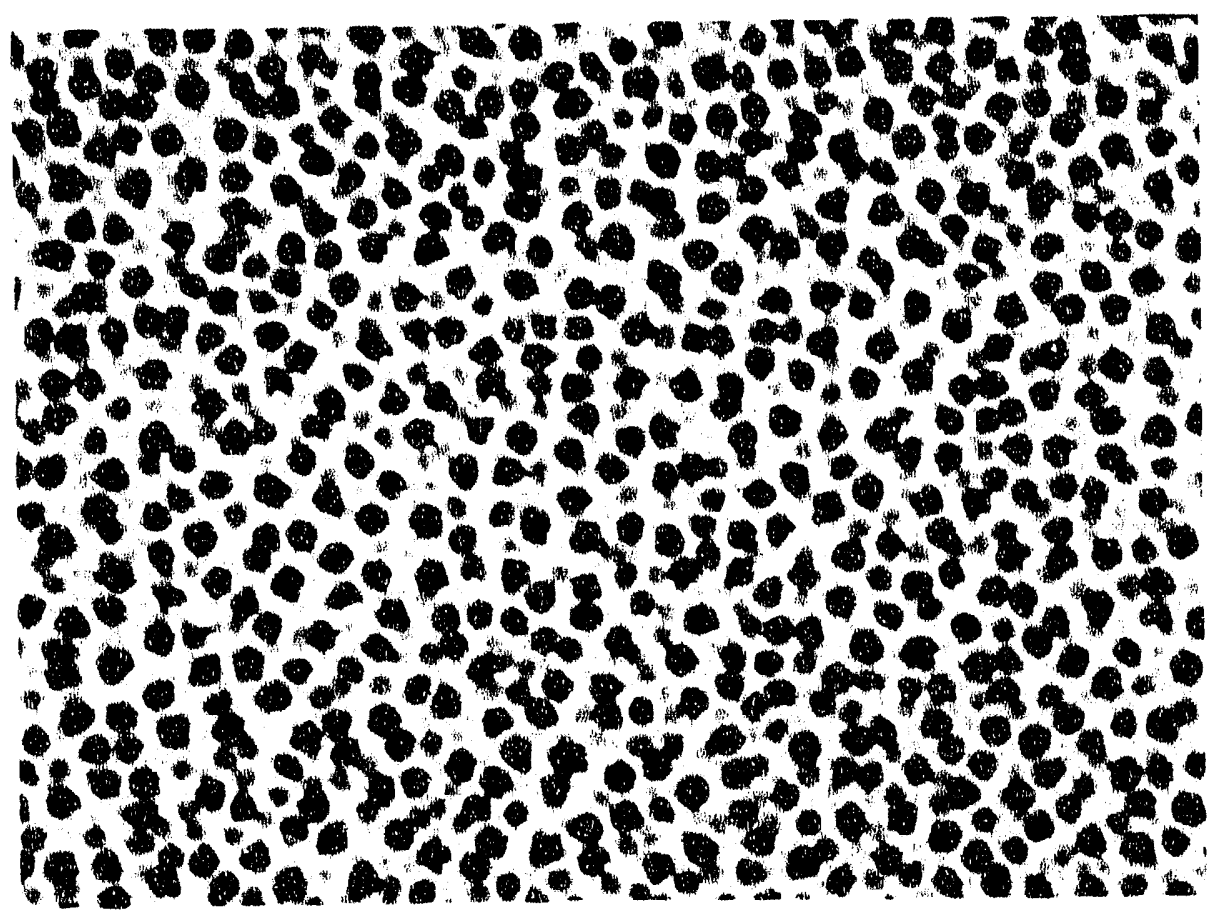

$662 \mathrm{~nm}$

Figure 3.2 SEM micrograph of surface of 0.2 micron pore size Anotec membrane. 


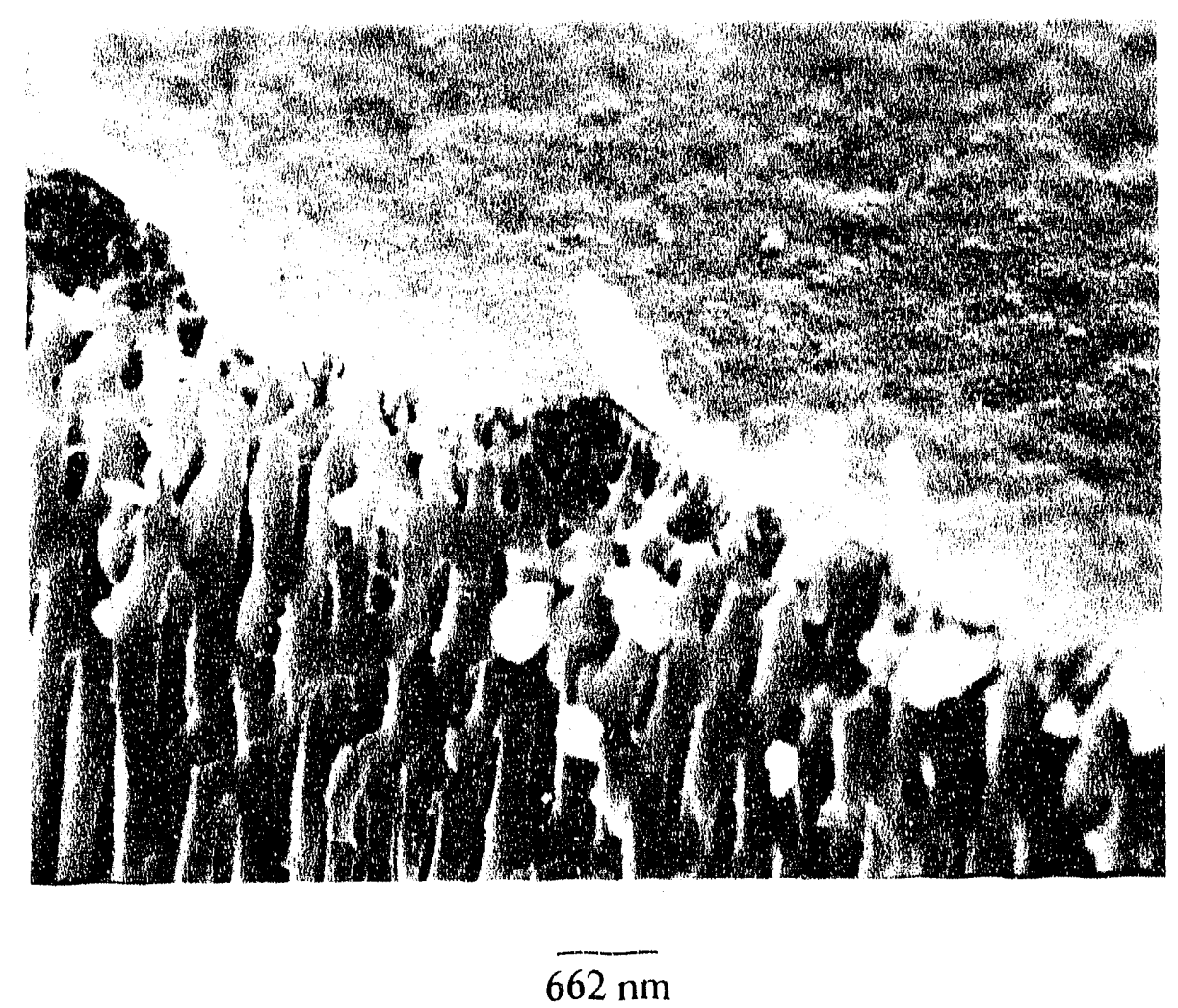

Figure 3.3 SEM micrograph of a fracture cross-section of a 0.02 micron pore size Anotec membrane coated with a zirconia film. The pores appear free of zircunia. 


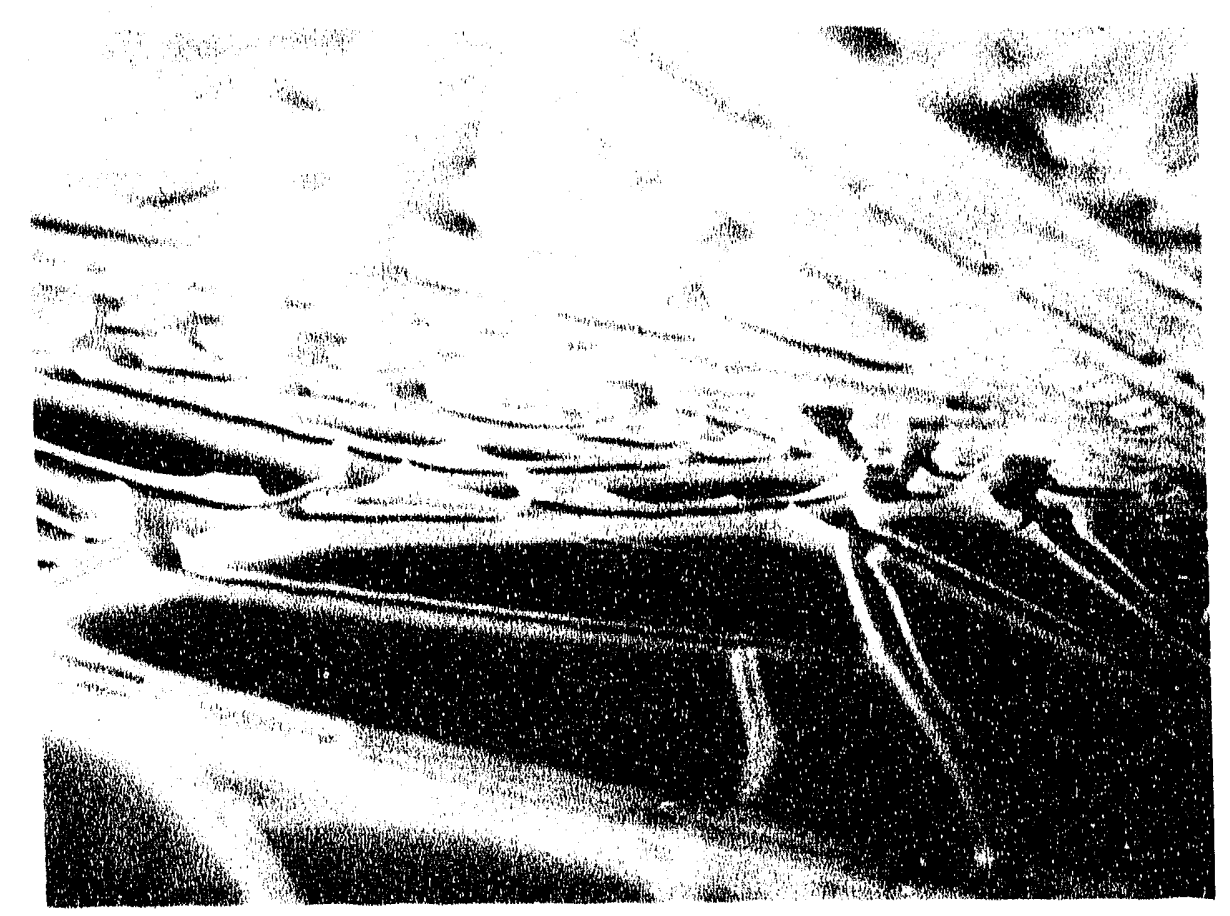

\section{$4.95 \mu \mathrm{m}$}

Figure 4.1 SEM high-angle view of a severely fractured zirconia film on a silicon substrate. Film "islands" can be seen to curl up at the edges, and the bare substrate can be seen between islands. 


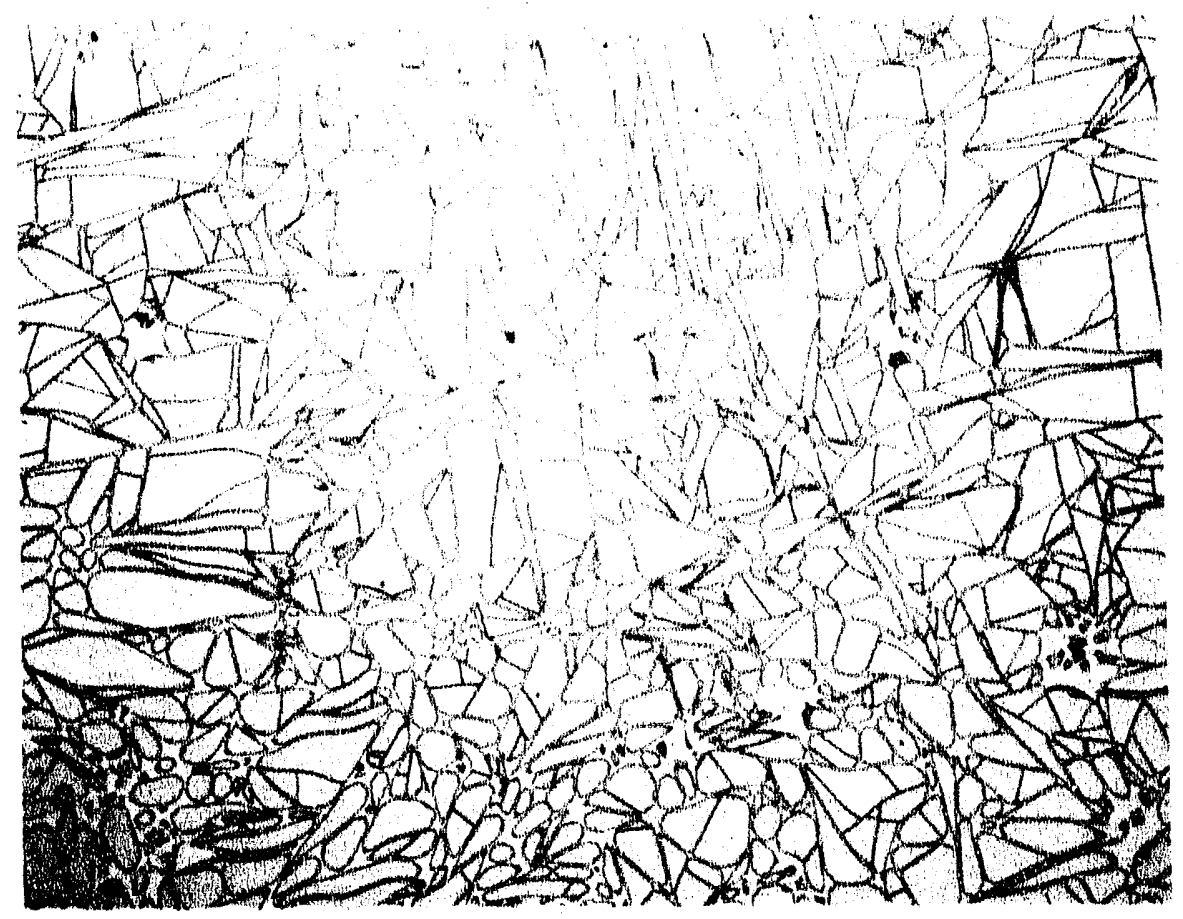

\section{$\overline{200 \mu \mathrm{m}}$}

Figure 4.2a Optical micrograph of a fractured zirconia film on a quartz substrate.

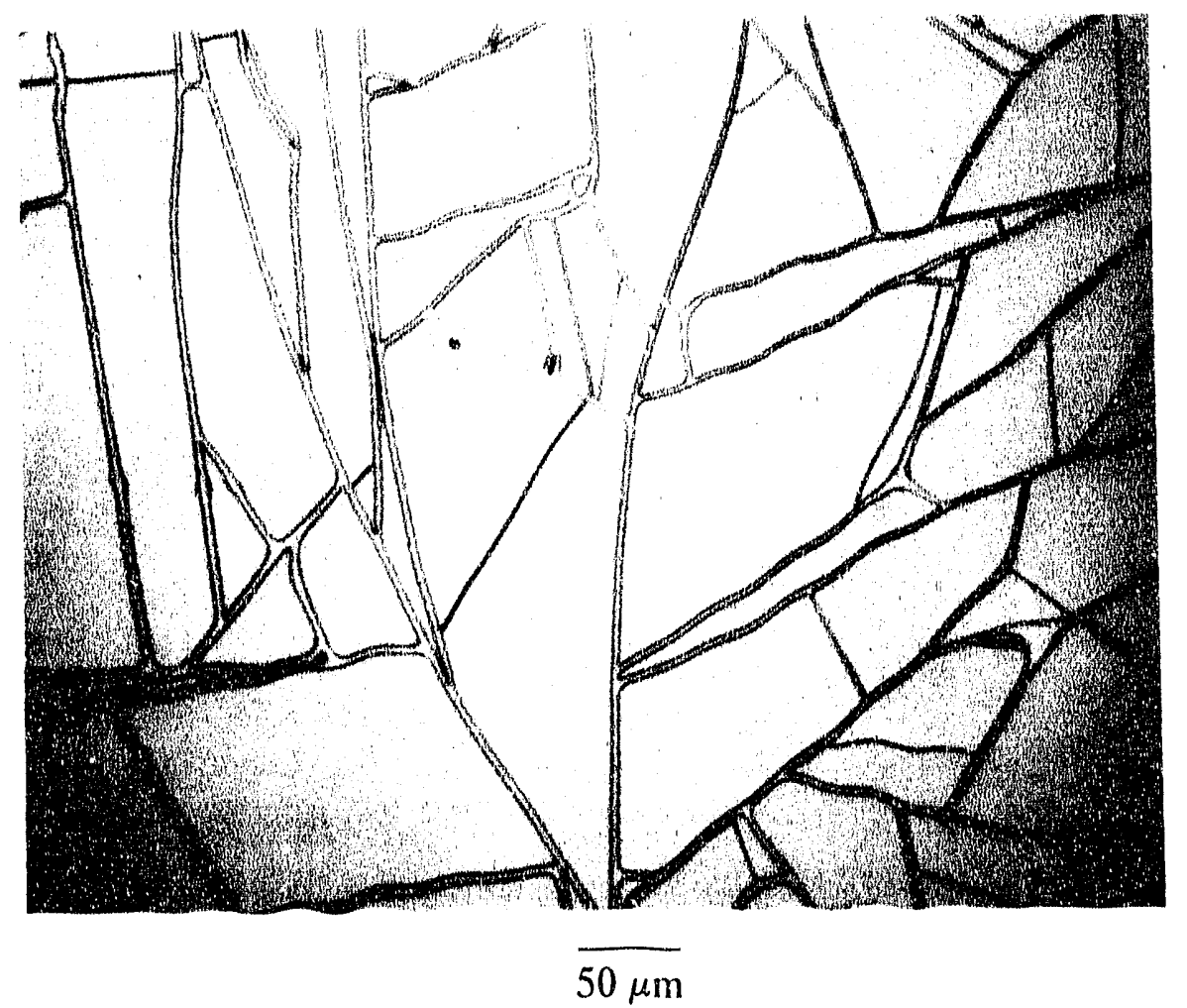

Figure 4.2b Higher magnification of above film. 


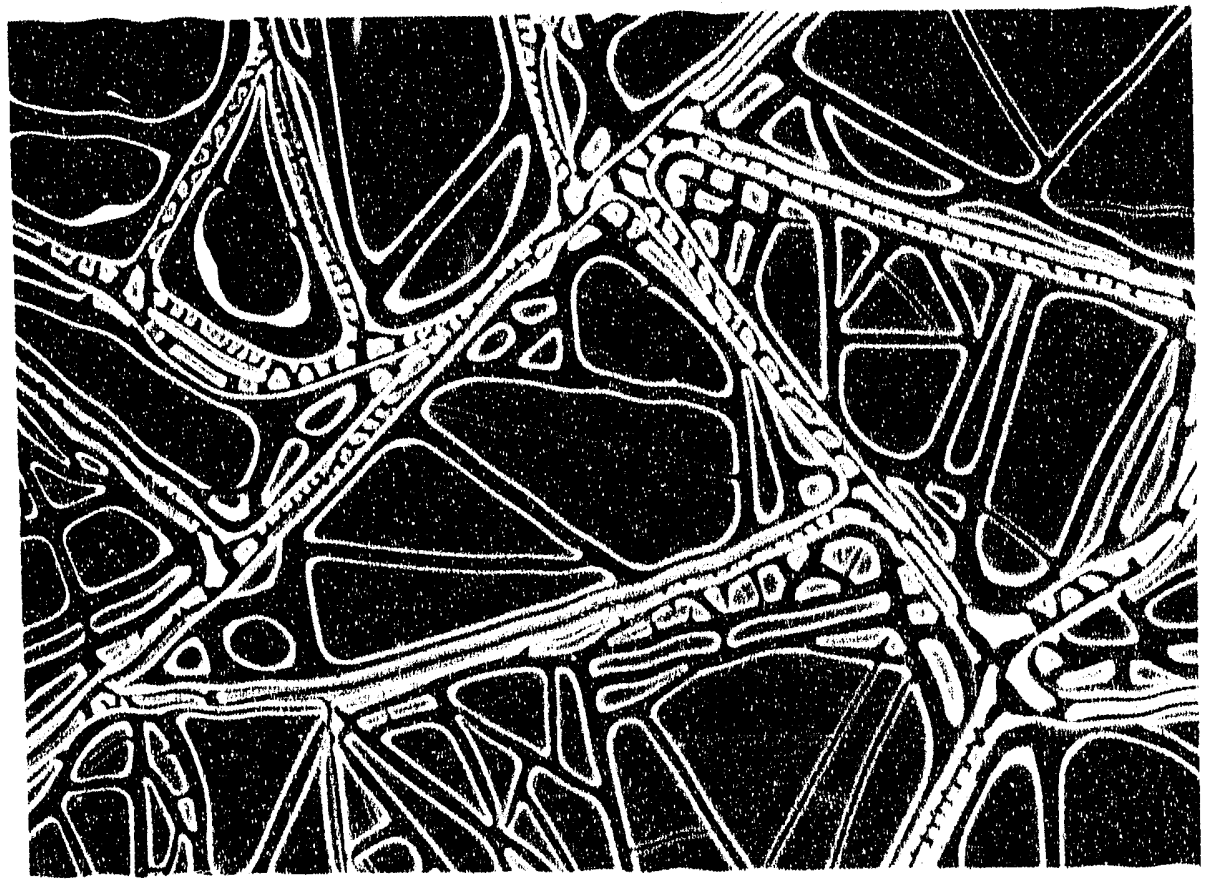

$\overline{16.6 \mu \mathrm{m}}$

Figure 4.3 SEM micrograph of a zirconia film on silicon after repeating the deposition and firing steps. A second island network can be seen on top of and between the original islands. 


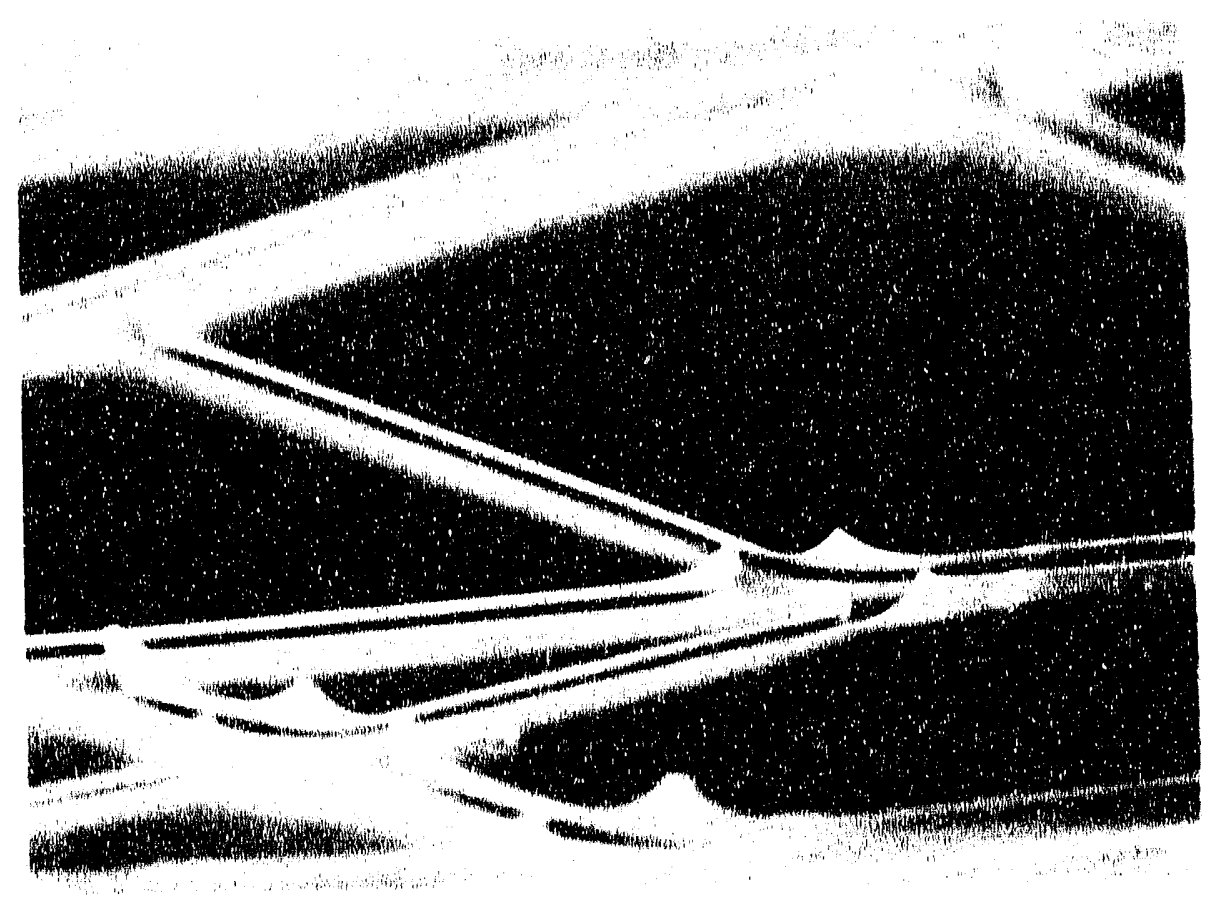

$2.48 \mu \mathrm{m}$

Figure 4.4 SEM high"angle view of a zirconia film on silicon. Film thickness can be estimated by viewing the cross-section at cracks. 


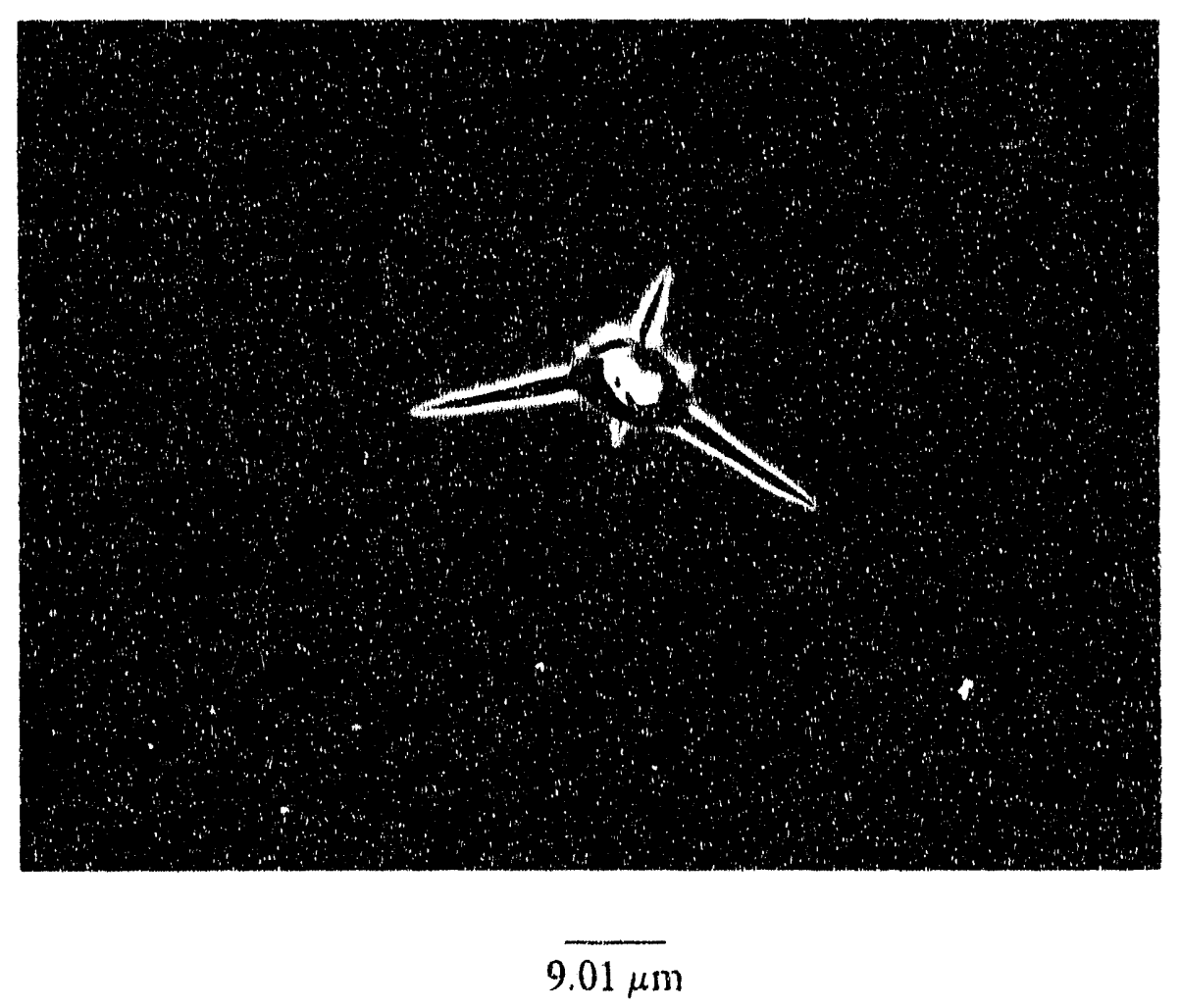

Figure 4.5 SEM micrograph of a defect-induced crack in a zirconia film on silicon. 


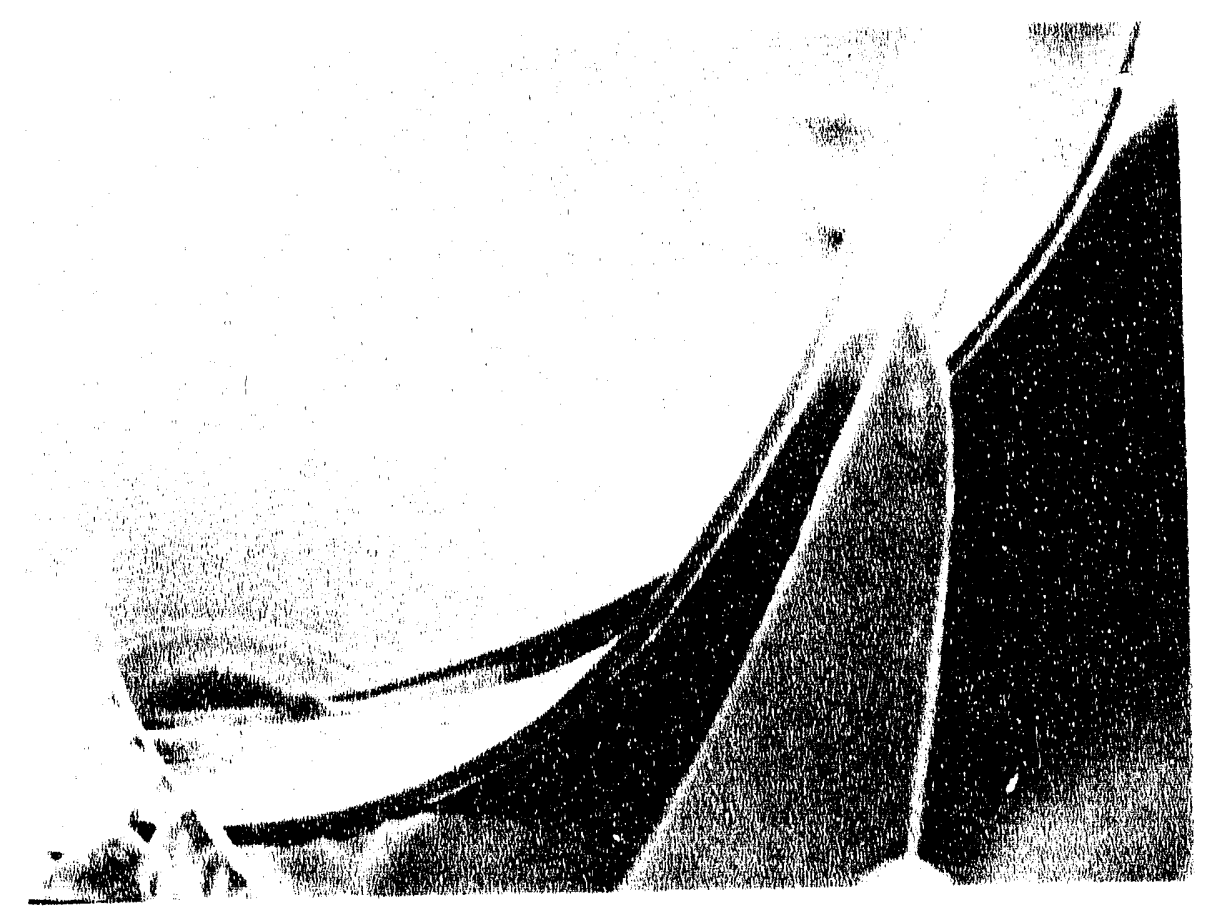

$10.0 \mu \mathrm{m}$

Figure 4.6 SEM side-view of a large delaminated zirconia film flake; a welldefined curvature can be seen. 


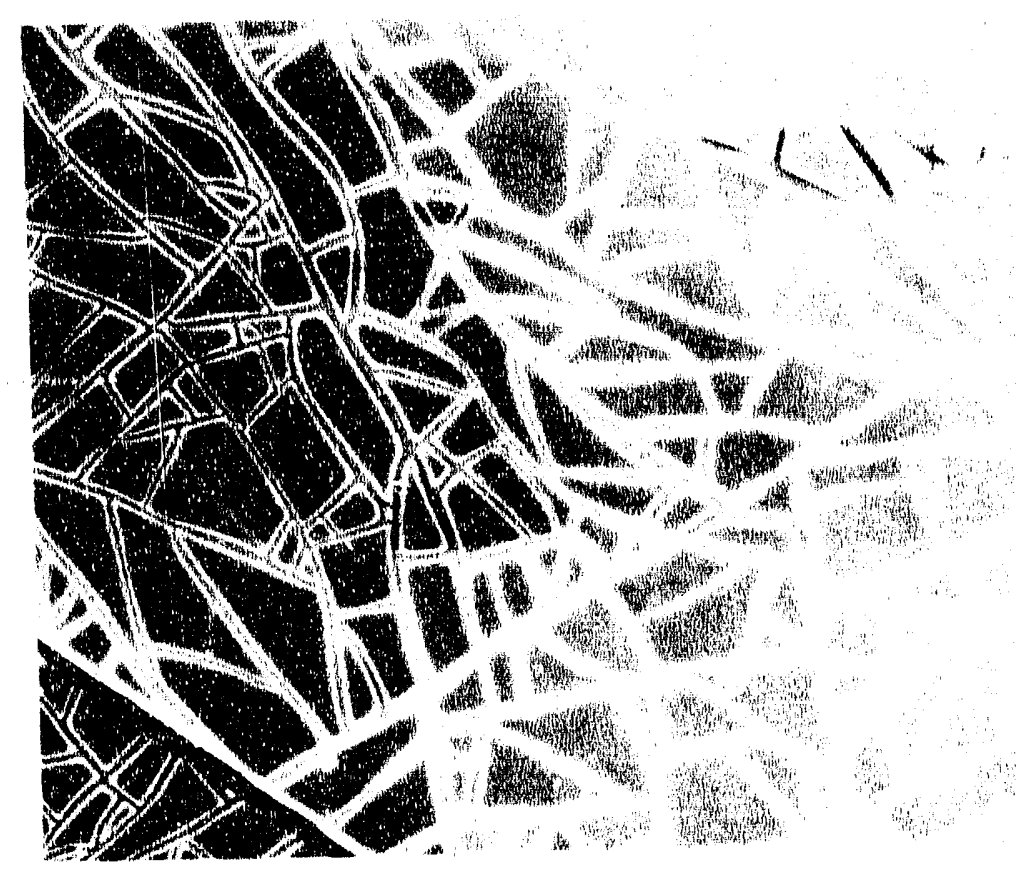

\section{$33 \mu \mathrm{m}$}

Figure 4.7a A thick zirconia film on a large-pore $(0.2 \mu \mathrm{m})$ Anotec membrane.

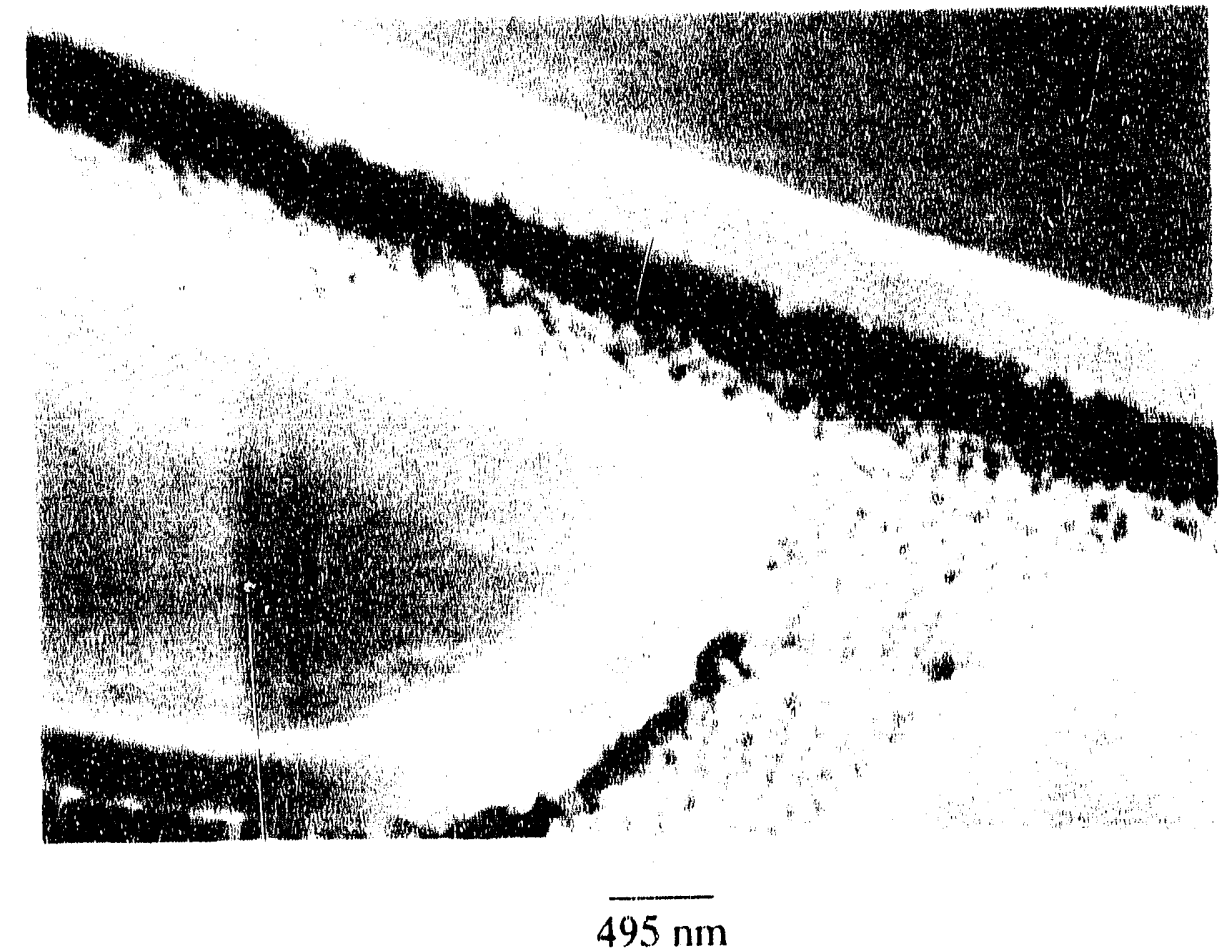

Figure 4.7b High-angle view of the above film at high magnification. 


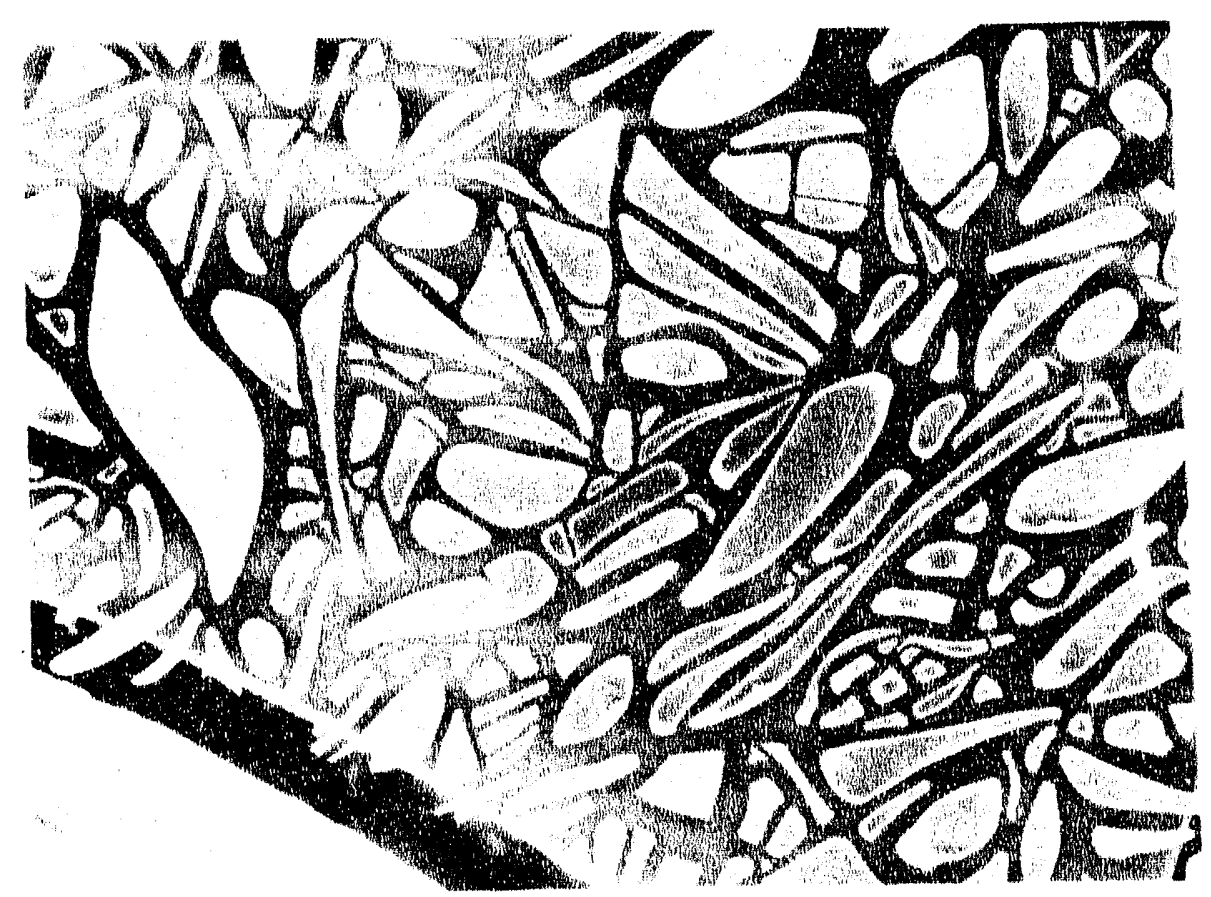

$\overline{33 \mu \mathrm{m}}$

Figure 4.8a A thick zirconia film on a small-pore $(0.02 \mu \mathrm{m})$ Anotec membrane.

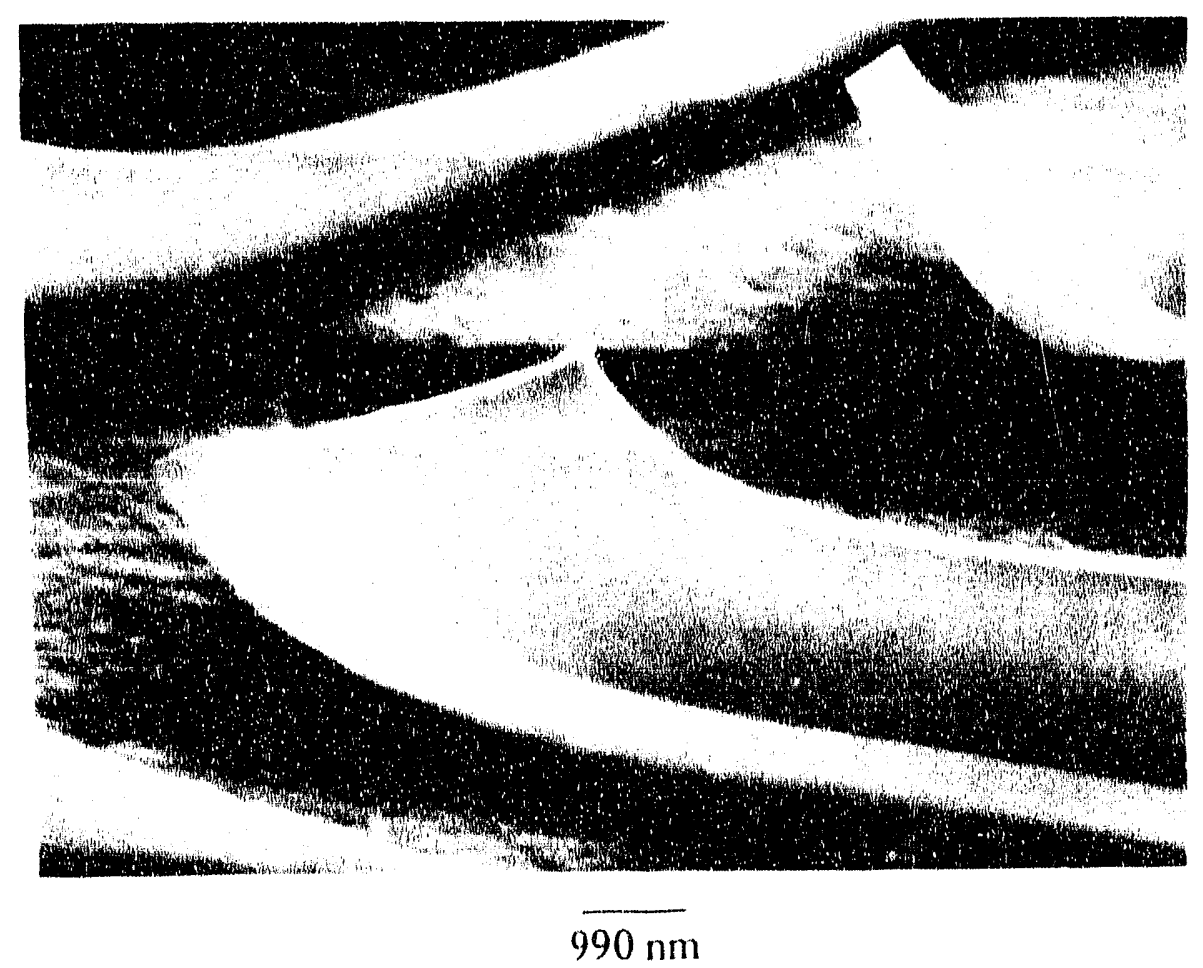

Figure 4.8b High-angle view of the above film at high magnification. 


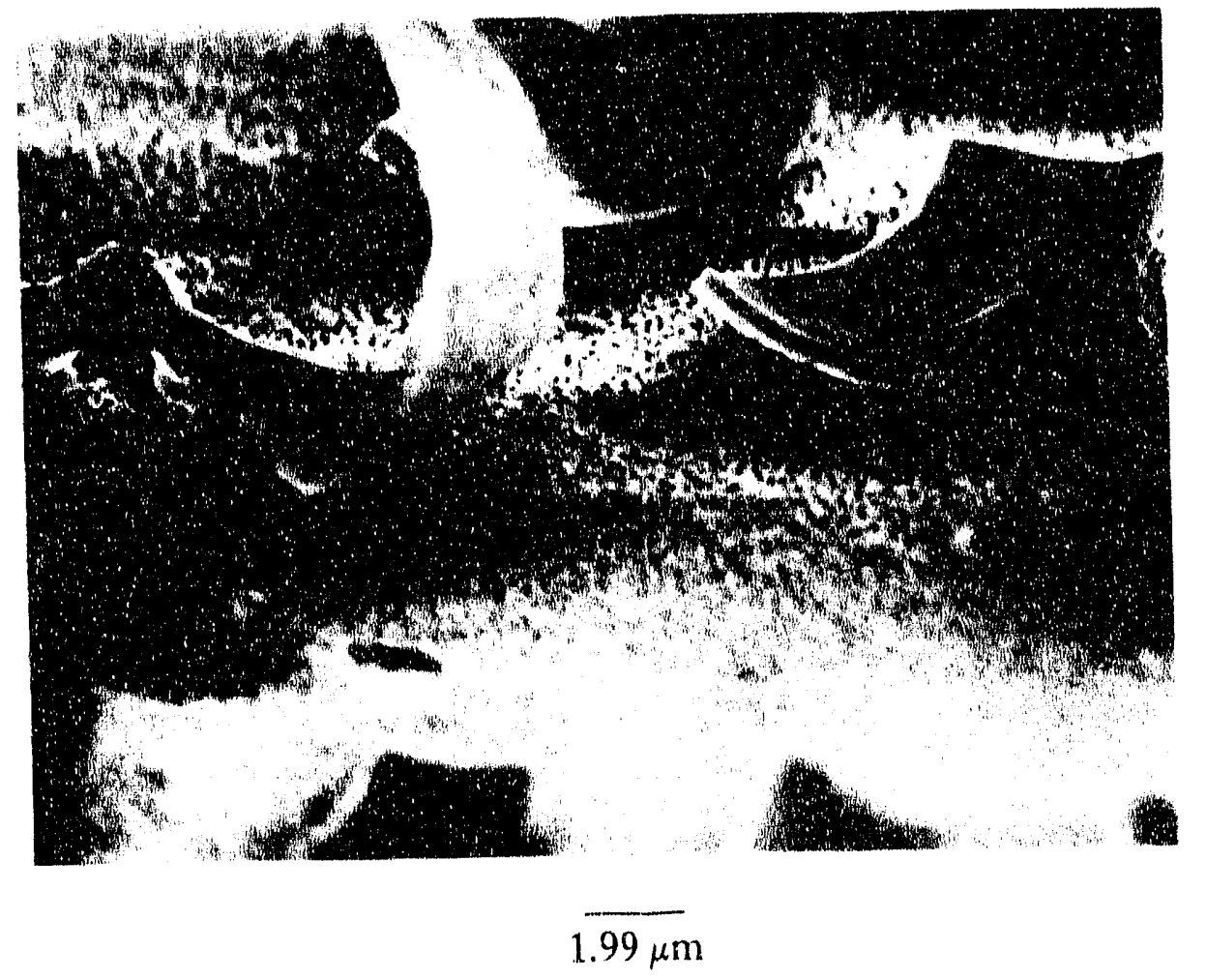

Figure 4.9 SEM high-angle view of a thick zirconia film on a $0.2 \mu \mathrm{m}$ pore size substrate; for this film thickness extensive delamination occurs. 


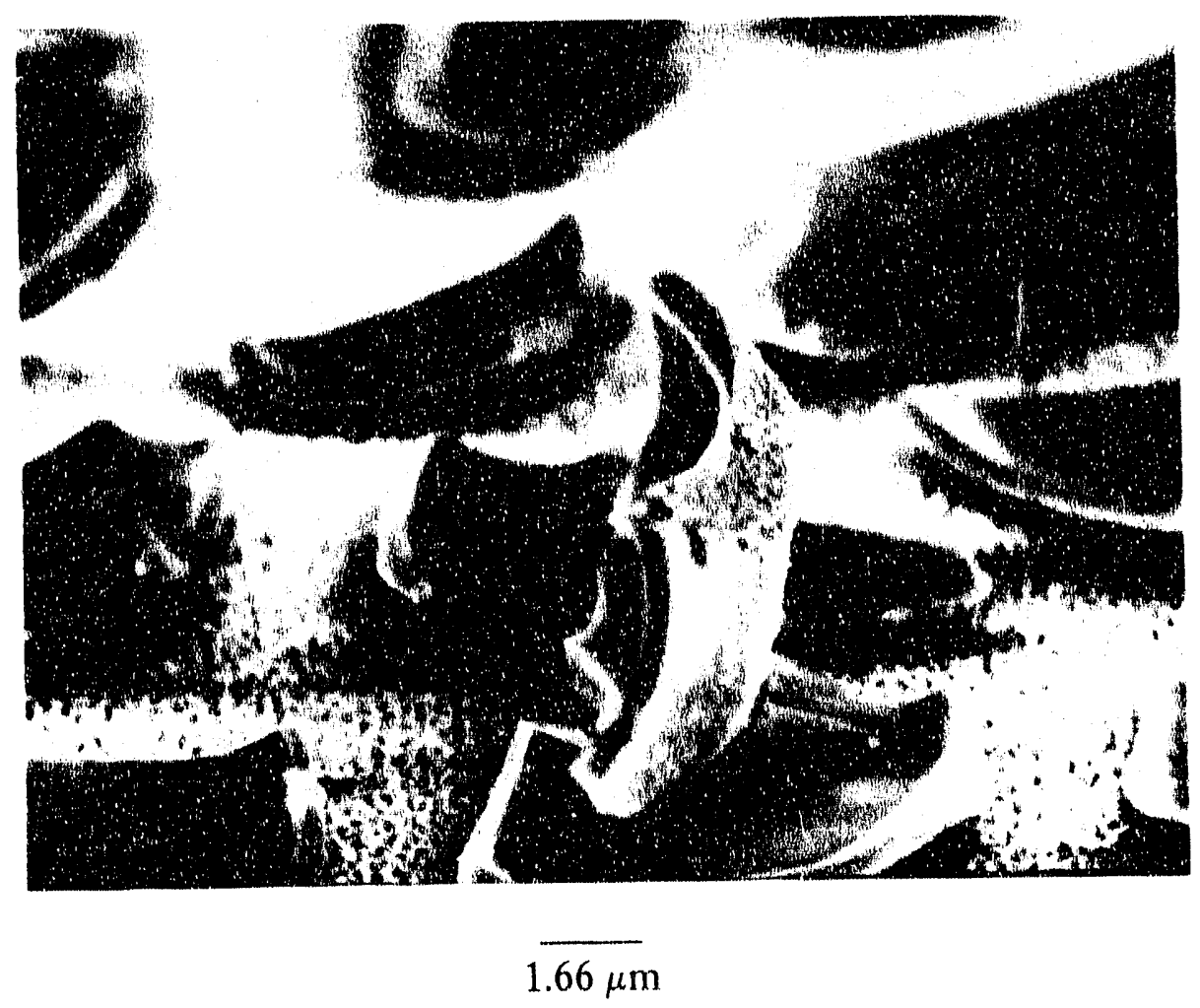

Figure 4.10 A completely separated section of the film from the preceding figure. Part of the substrate adhered to the film, and at the center the crack went very deep into the substrate; the columnar structure can be seen propping up the delaminated flake. 


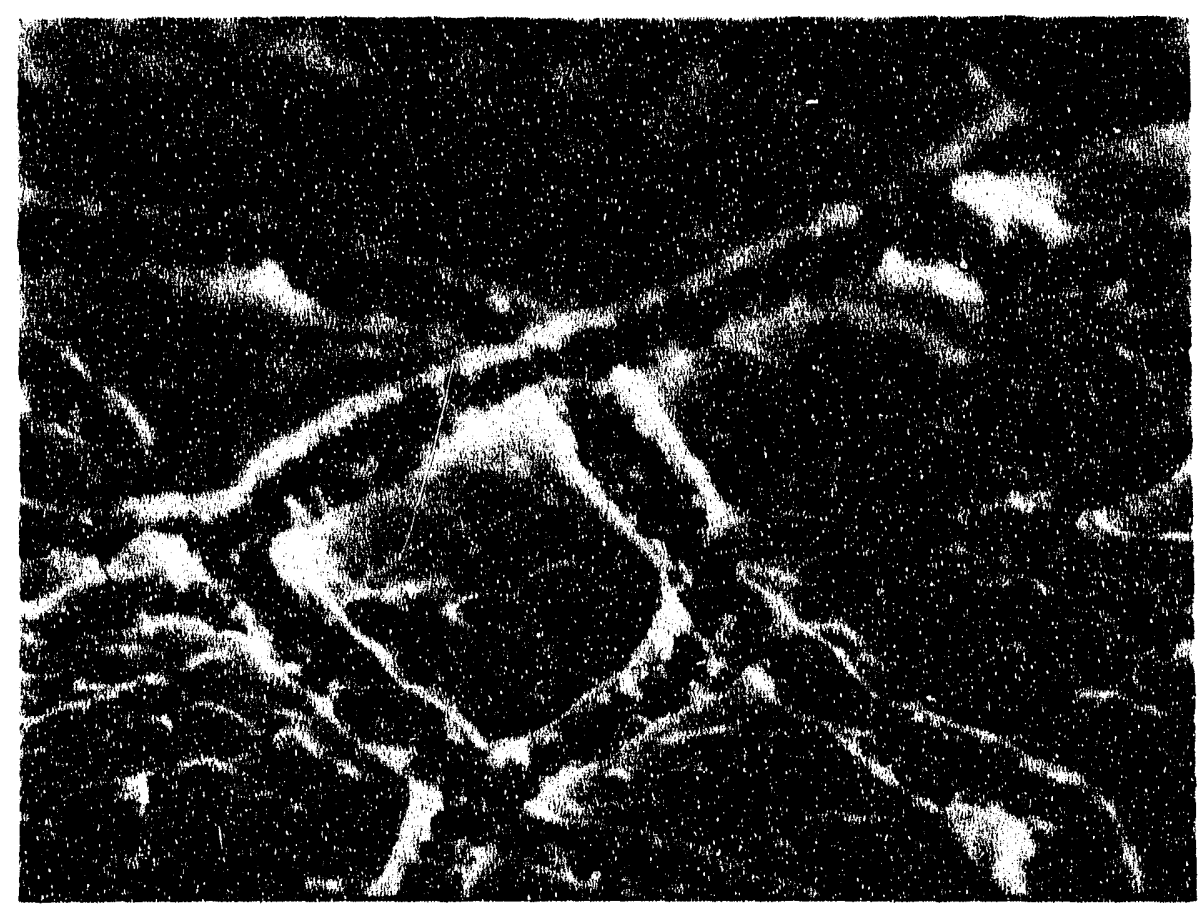

$662 \mathrm{~nm}$

Figure 4.11 High-angle SEM view of a zirconia film on a $0.2 \mu \mathrm{m}$ pore size substrate. Localized cracking can be seen within the islands left by unstable cracking. 


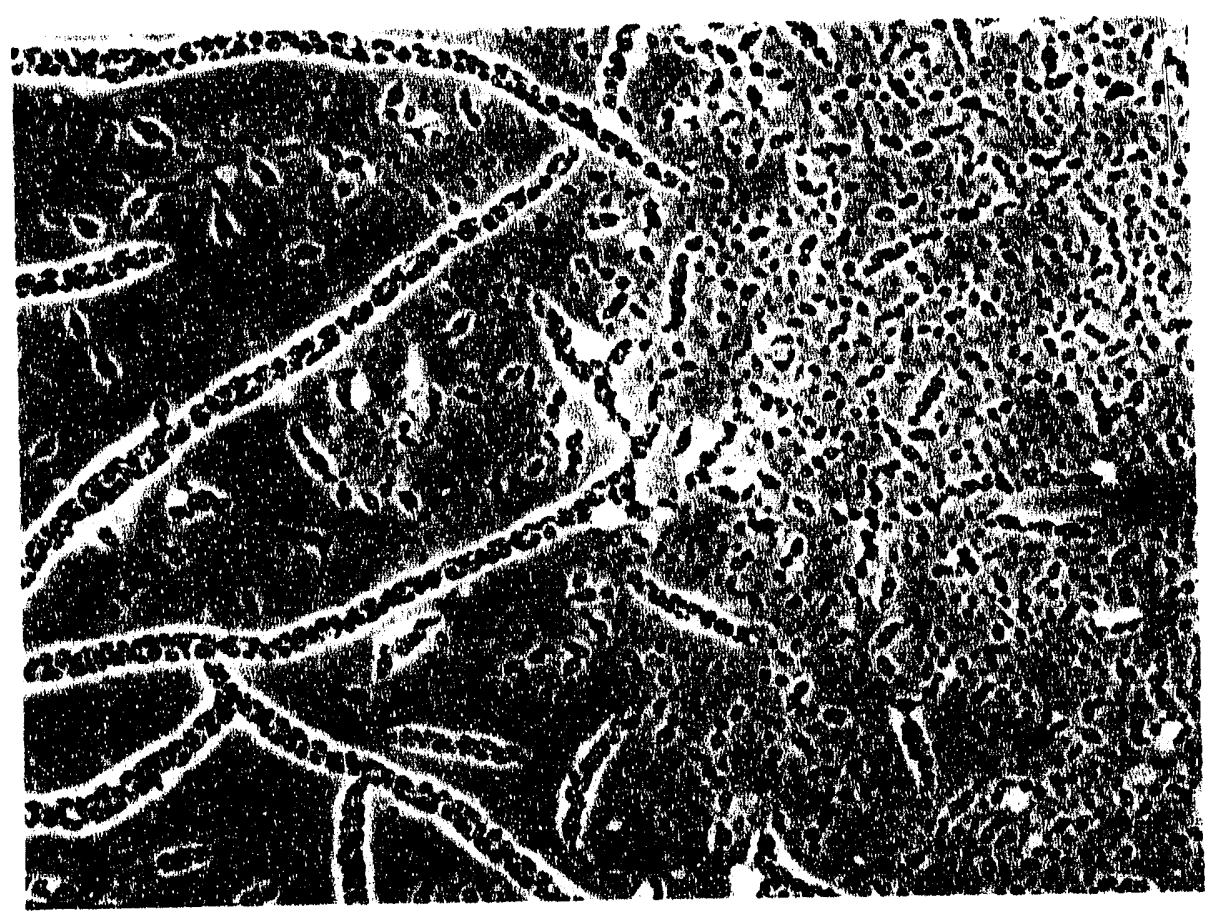

$1.99 \mu \mathrm{m}$

Figure 4.12 SEM view of a zirconia film on a $0.2 \mu \mathrm{m}$ pore size substrate in a film thickness transition region. Cracking behavior changes as the film gets thinner (from left to right). 


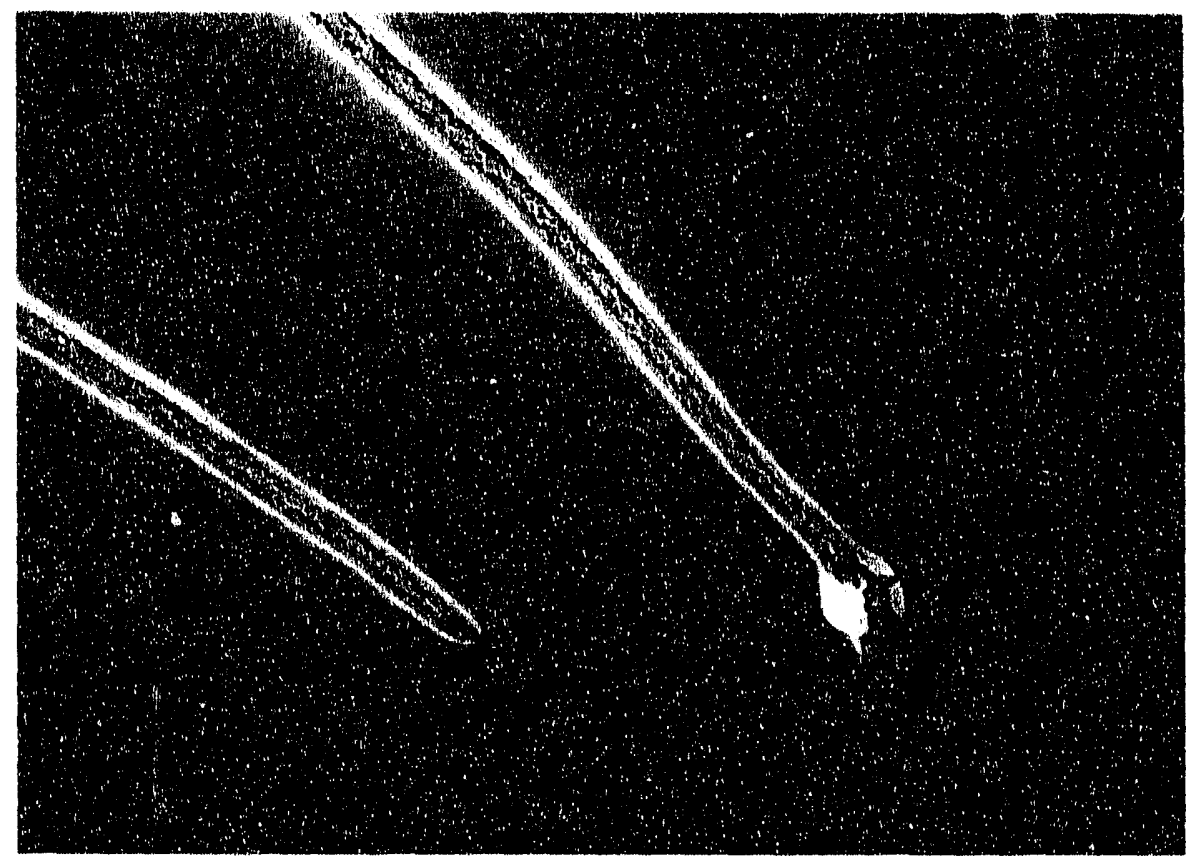

\section{$\overline{1.99 \mu \mathrm{m}}$}

Figure 4.13 SEM view of a zirconia film on a $0.02 \mu \mathrm{m}$ pore size substrate in a film thickness transition region. Cracks end as they enter the thinner region from the thicker region at the upper left. 


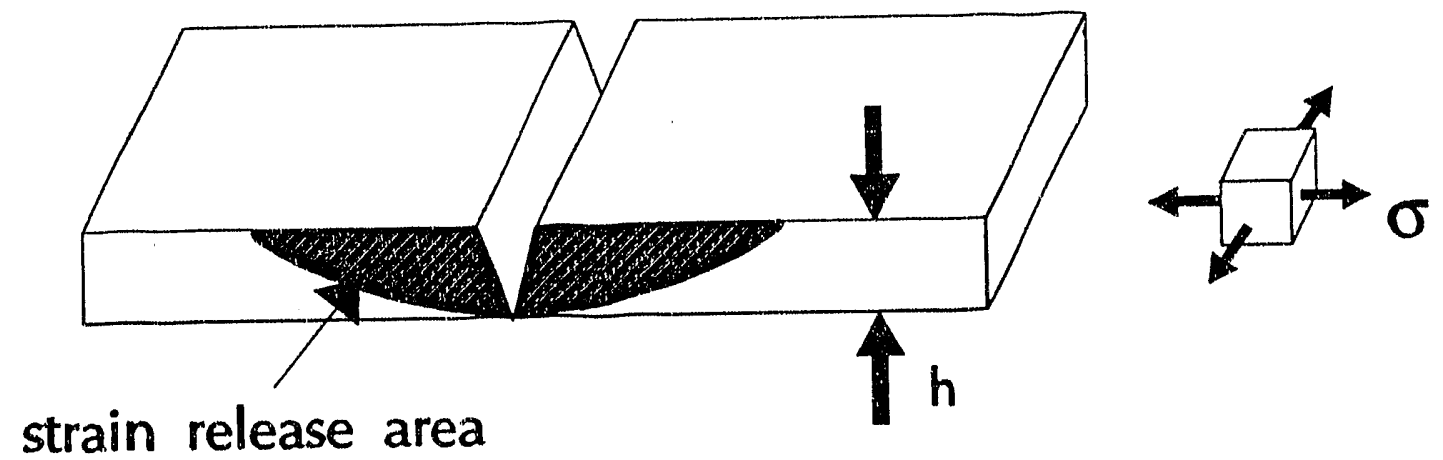

$$
\mathrm{K}_{\mathrm{l}}=0.77 \sigma \sqrt{\pi h_{\mathrm{C}}}
$$

Figure 4.14 A model of the strain release area around a crack in a thin film. The film thickness $h$ represents the maximum effective length of the crack if strain release volume is considered as a function of crack length. 


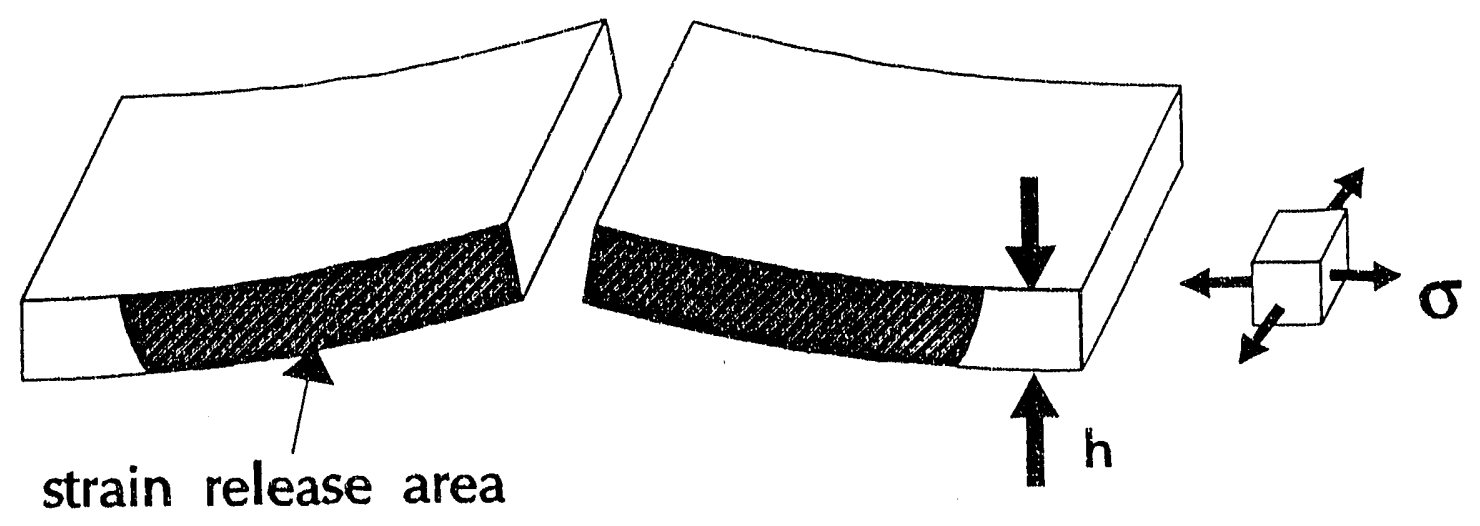

$$
\mathrm{K}_{\mathrm{l}}<0.77 \sigma \sqrt{\pi h_{\mathrm{c}}}
$$

Figure 4.15 The strain release area around a crack in a thin film which undergoes some delamination. The strain release volume per new crack surface area is greater here than in the previous figure; the $h_{c}$ value from the adherent-film geometry will give a stress intensity which in this case exceeds the fracture toughness of the material. 

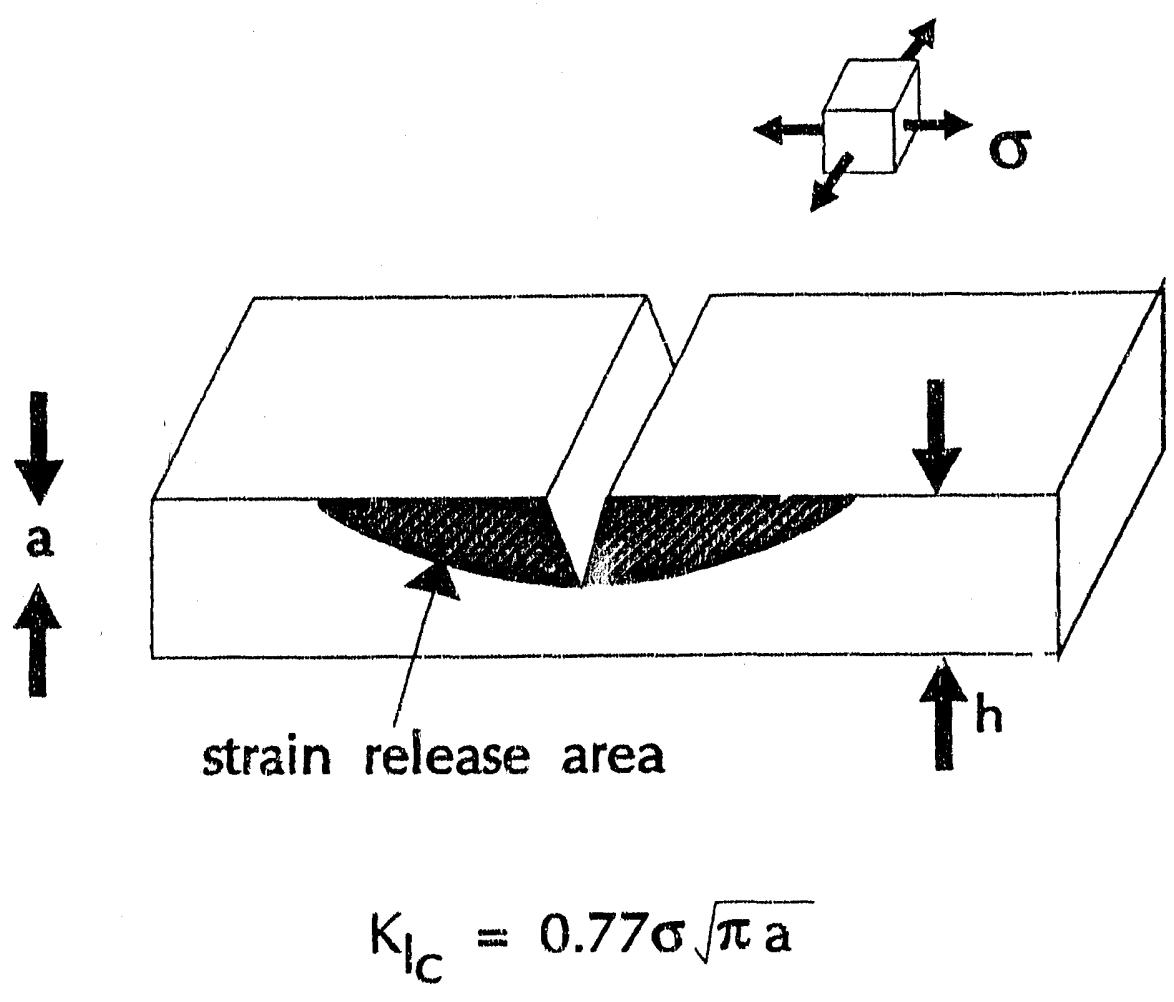

Figure 4.16 For films thicker than $h_{C}$, the flaw size a plays the same role as the film thiickness in Figure 4.14. However, in this case the crack is growing in a direction normal to the plane of the film rather than in the plane of the film. 


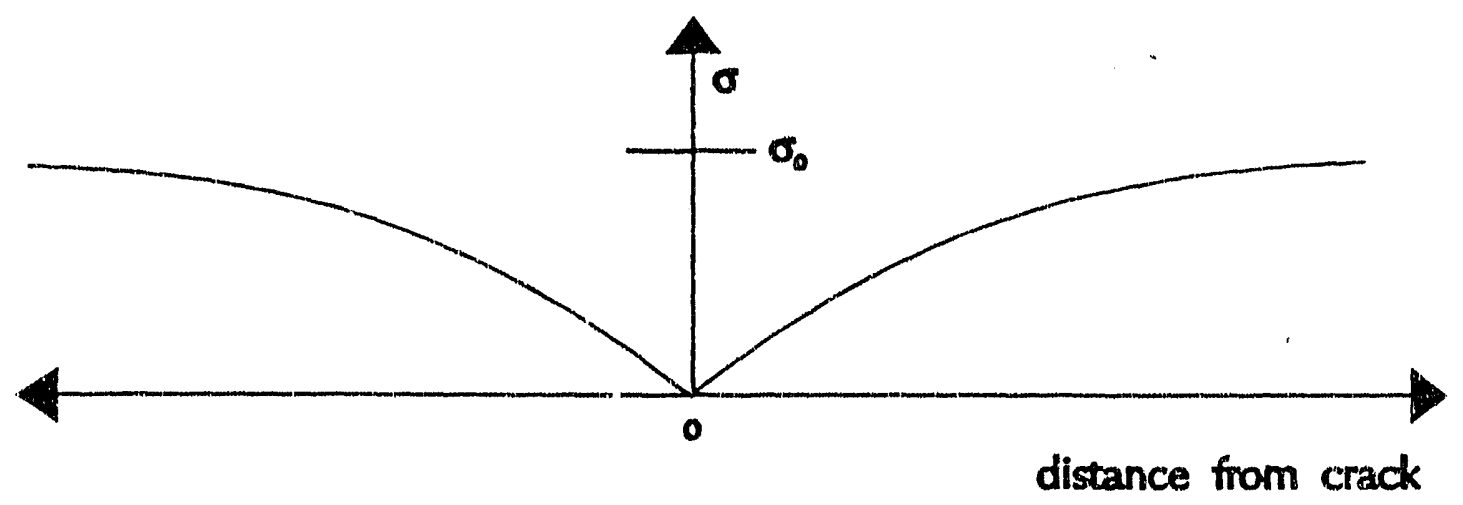

Figure 4.17 A diagram of the stress distribution around a crack in a thin film; "strain release" is a gradual function of distance from the crack. 

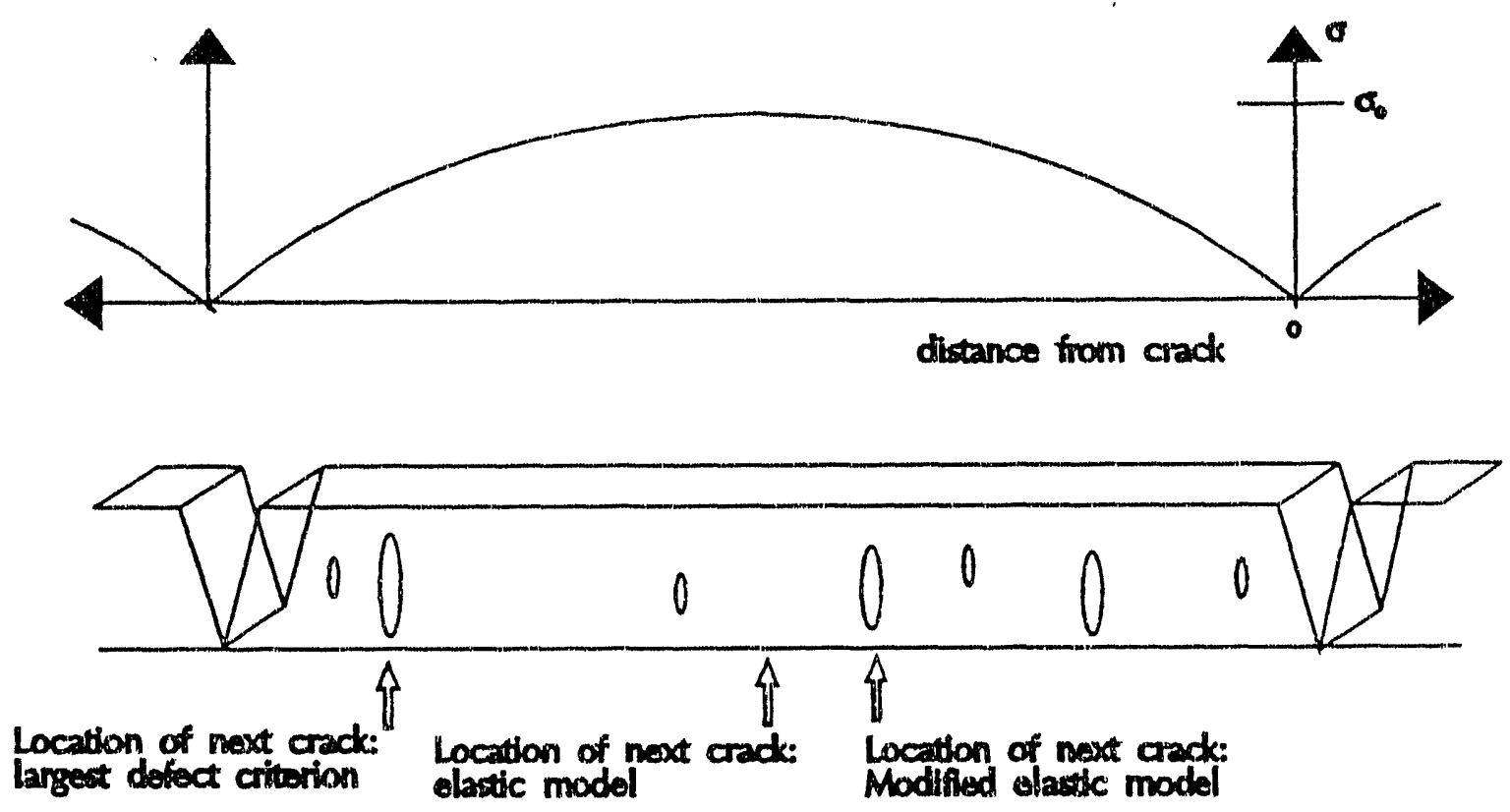

Figure 4.18 The stress distribution between two cracks in a thin film. The location of the next crack is determined not solely by the largest defect present or by the location of the largest remaining stress, but rather by the most favorable combination of these two parameters. 


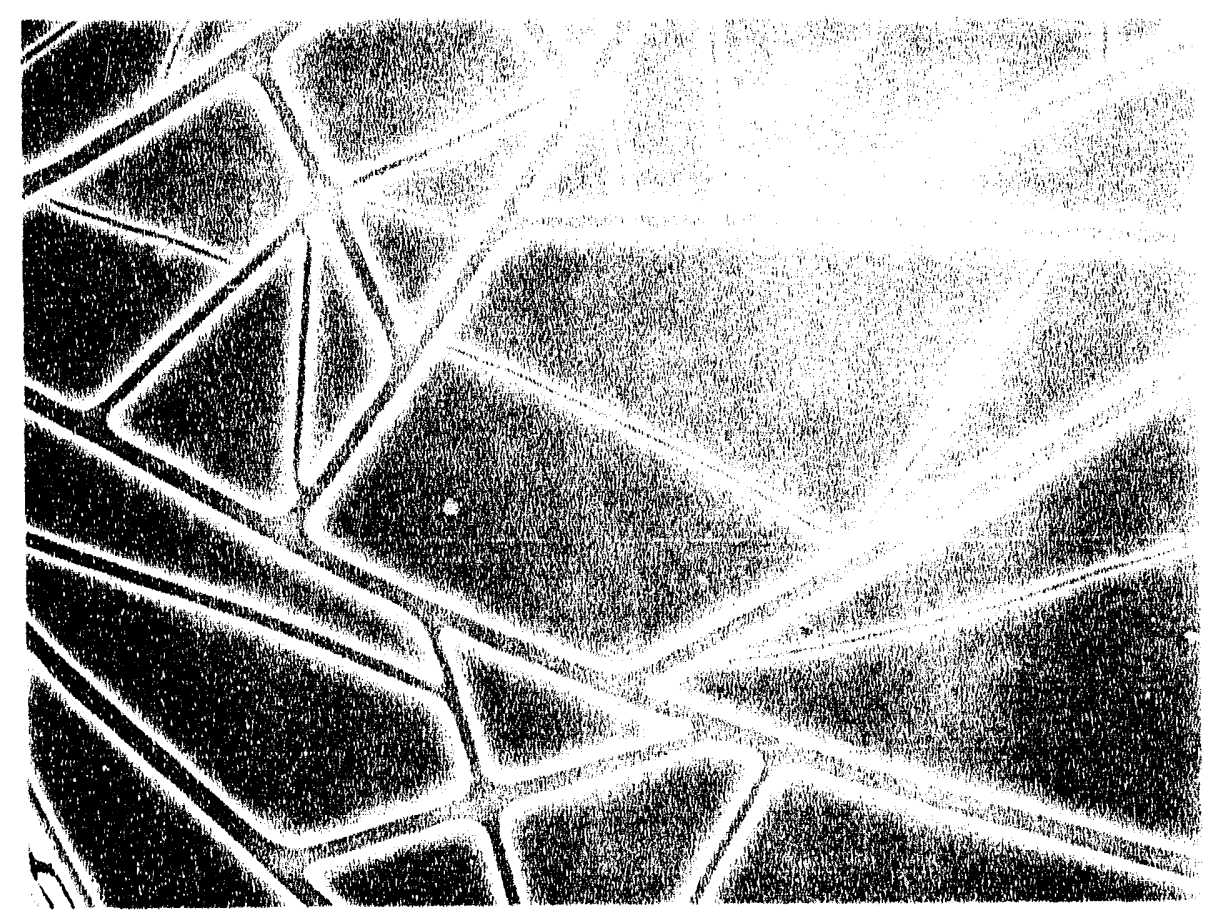

\section{$\overline{662 \mathrm{~nm}}$}

Figure 4.19 SEM micrograph of a crack pattern in a zirconia film on silicon. Laterstage cracks (smaller width cracks) can be seen to arrest when they come close enough to an existing crack. 


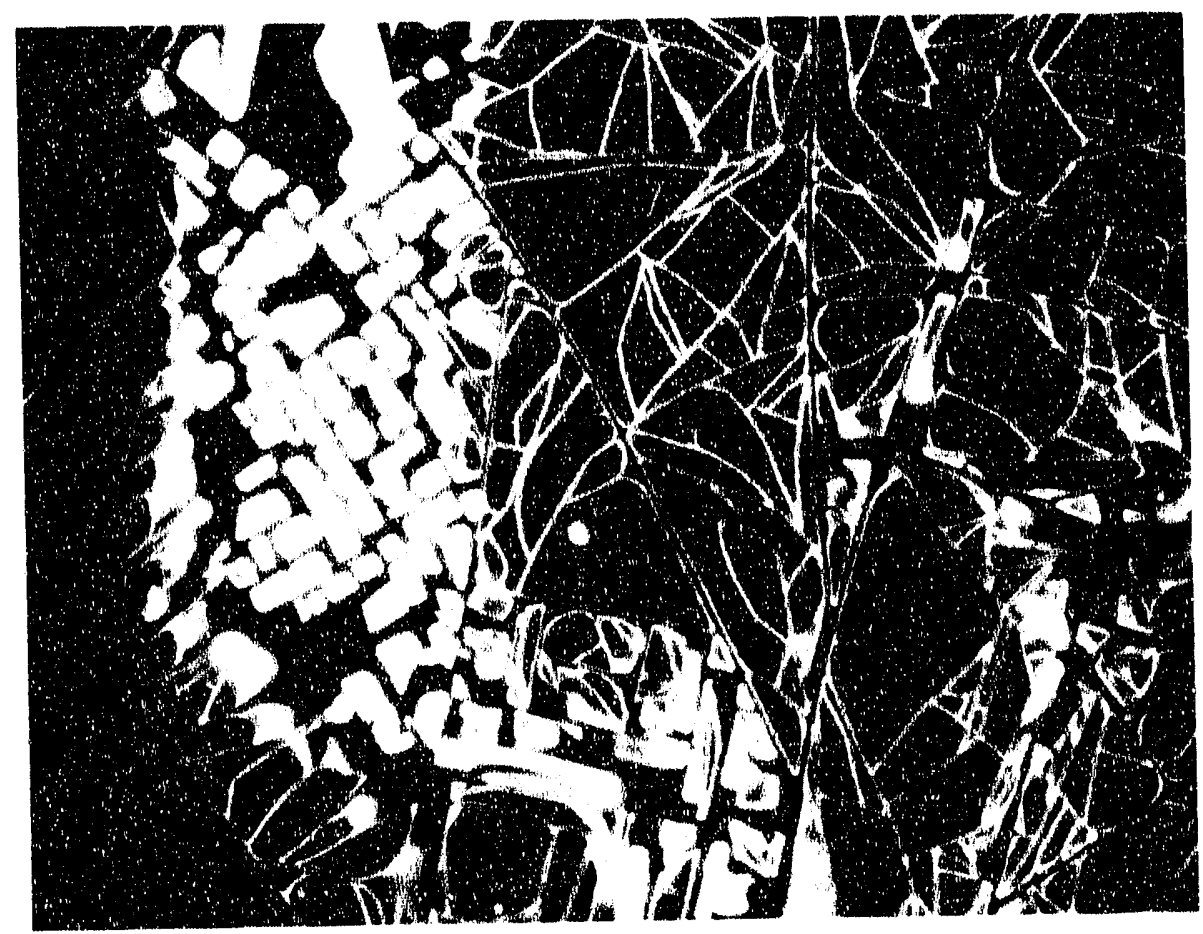

$\overline{662 \mathrm{~nm}}$

Figure 4.20 Optical micrograph (with crossed polarizers) of a crack pattern at the edge of a zirconia coating on silicon (uncoated substrate can be seen at the left). Cooperative cracking can be seen in a thick border region along the film edge. 


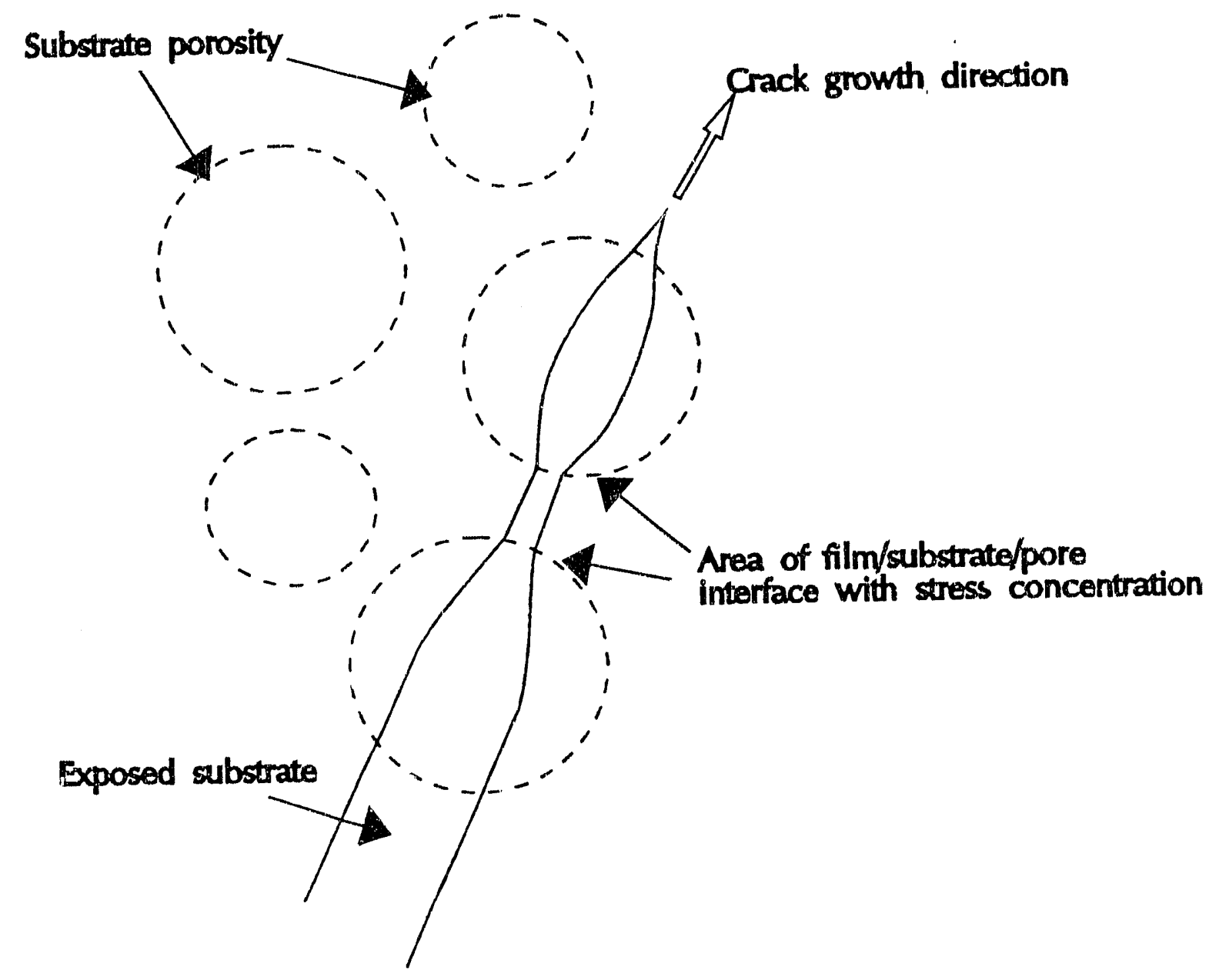

Figure 4.21 Model of a growing crack on a porous substrate. Stresses may concentrate at the pore wall/film interface. 


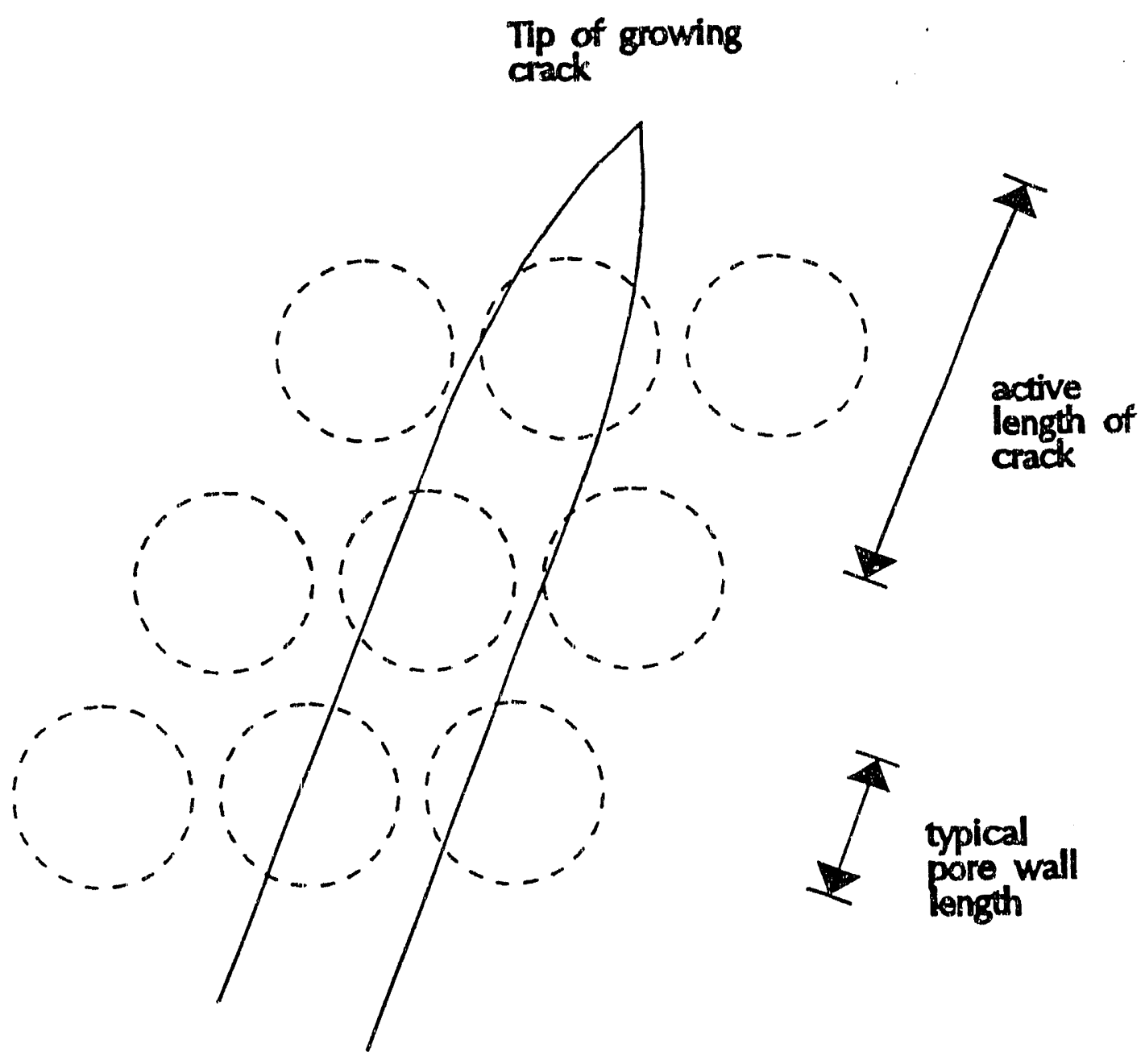

Figure 4.22 Model of a growing crack on a porous substrate, in which the active length of crack is greater than the typical interpore spacing. 
Film Thickness (microns)

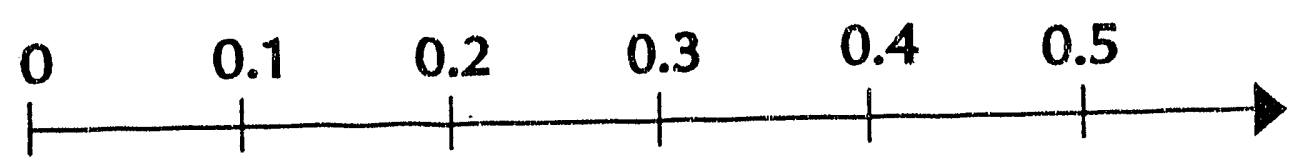

Continuous Substrates:

50\% Porous Substrates:

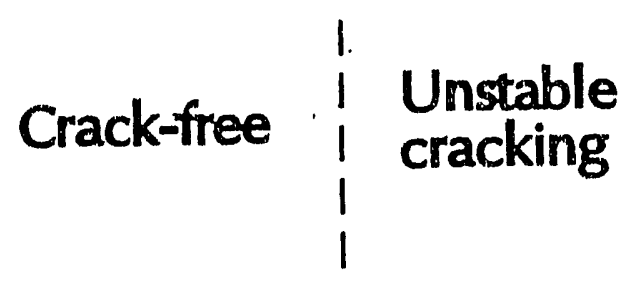

\begin{tabular}{l|l|l}
$\begin{array}{l}\text { No unstable } \\
\text { cracking }\end{array}$ & 0 & $\begin{array}{l}\text { Always } \\
\text { unstable }\end{array}$ \\
\cline { 2 - 3 } & $\begin{array}{l}\text { Increasing probability } \\
\text { that crack will cross } \\
\text { pore wall }\end{array}$ & cracking
\end{tabular}

\begin{tabular}{l|l} 
Pore-induced cracking: & $\begin{array}{l}\text { No pore-induced } \\
\text { cracking: } 0.2\end{array}$ \\
0.2 micron pores & micron pores
\end{tabular}

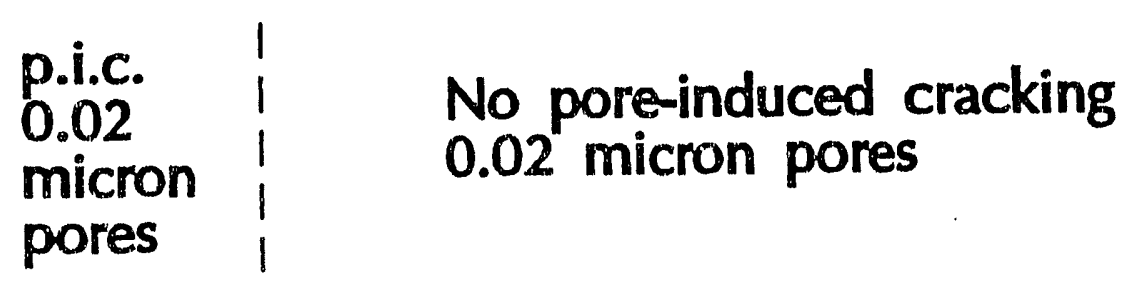

Figure 4.23 Outline of thickness dependence of cracking for various substrates. A crack-free range exists for $0.02 \mu \mathrm{m}$ substrates from approx. 0.1 to $0.2 \mu \mathrm{m}$ thicknesses. 


\section{Large pores, small film thickness:}

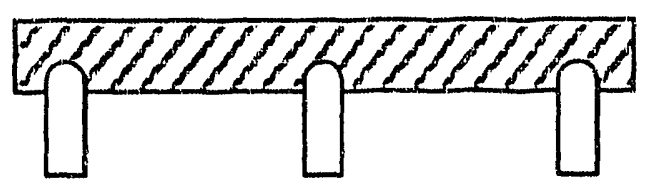

Stress

Concentration Model:

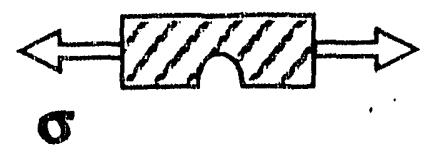

Small pores, large film thickness:

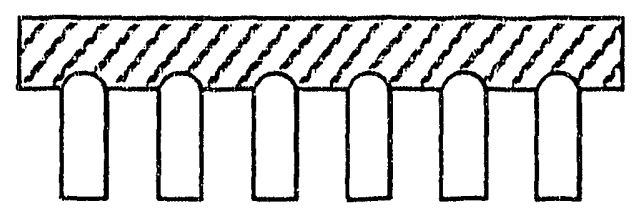

Stress

Concentration Model:

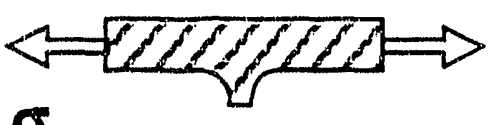

Figure 4.24 Stress concentration models for different film/substrate geometries. When the pore walls are placed further apart, a crack-like defect may be introduced, while closely spaced pore walls do not allow the stresses to be distributed throughout the thickness of the film, leaving only ridge-like defects, a less severe stress state. Stress concentrations are maximized for thinner films. 


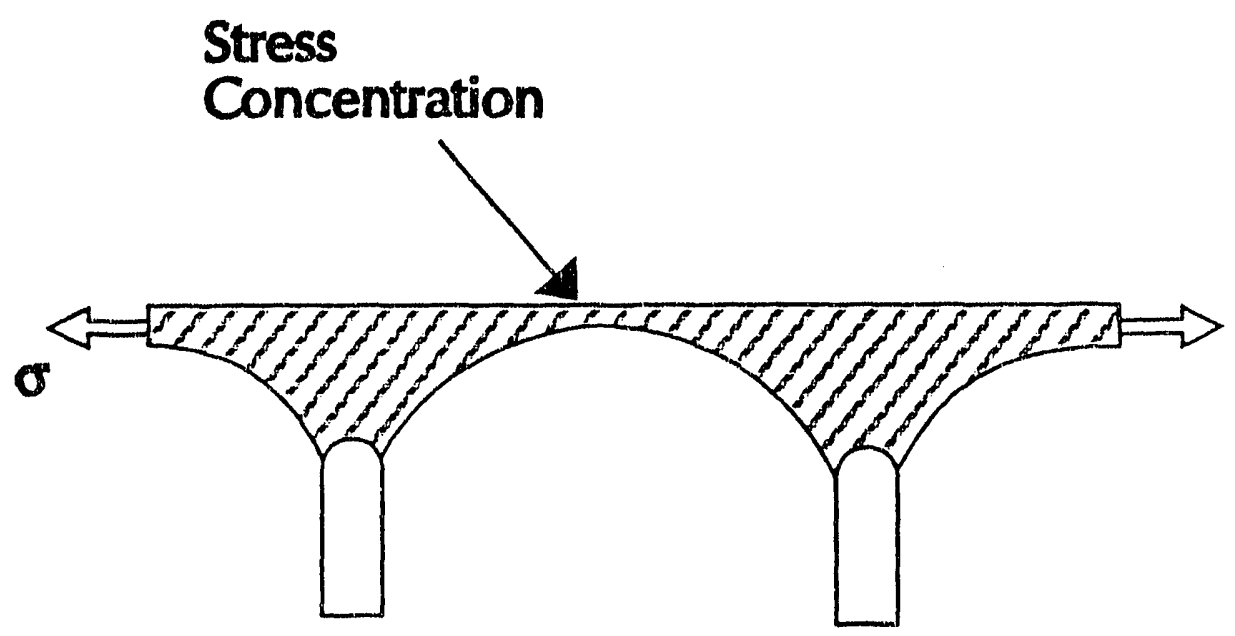

Figure 4.25 Model of the film geometry that would occur if the wetting angle of the liquid coating solution is maintained after drying. Stress concentrations over the pores would develop for sufficiently thin films. 


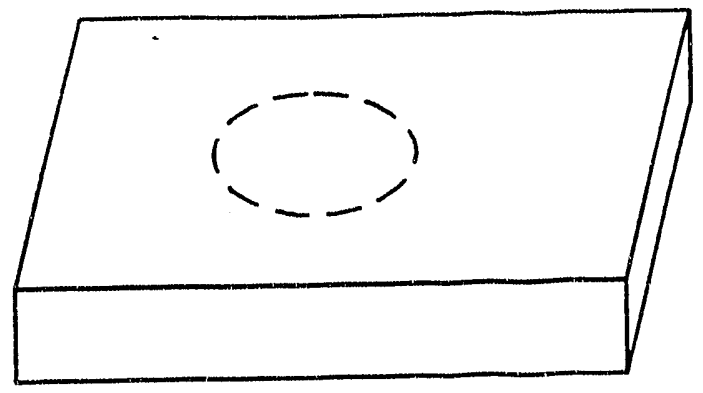

\section{(film over pore)}
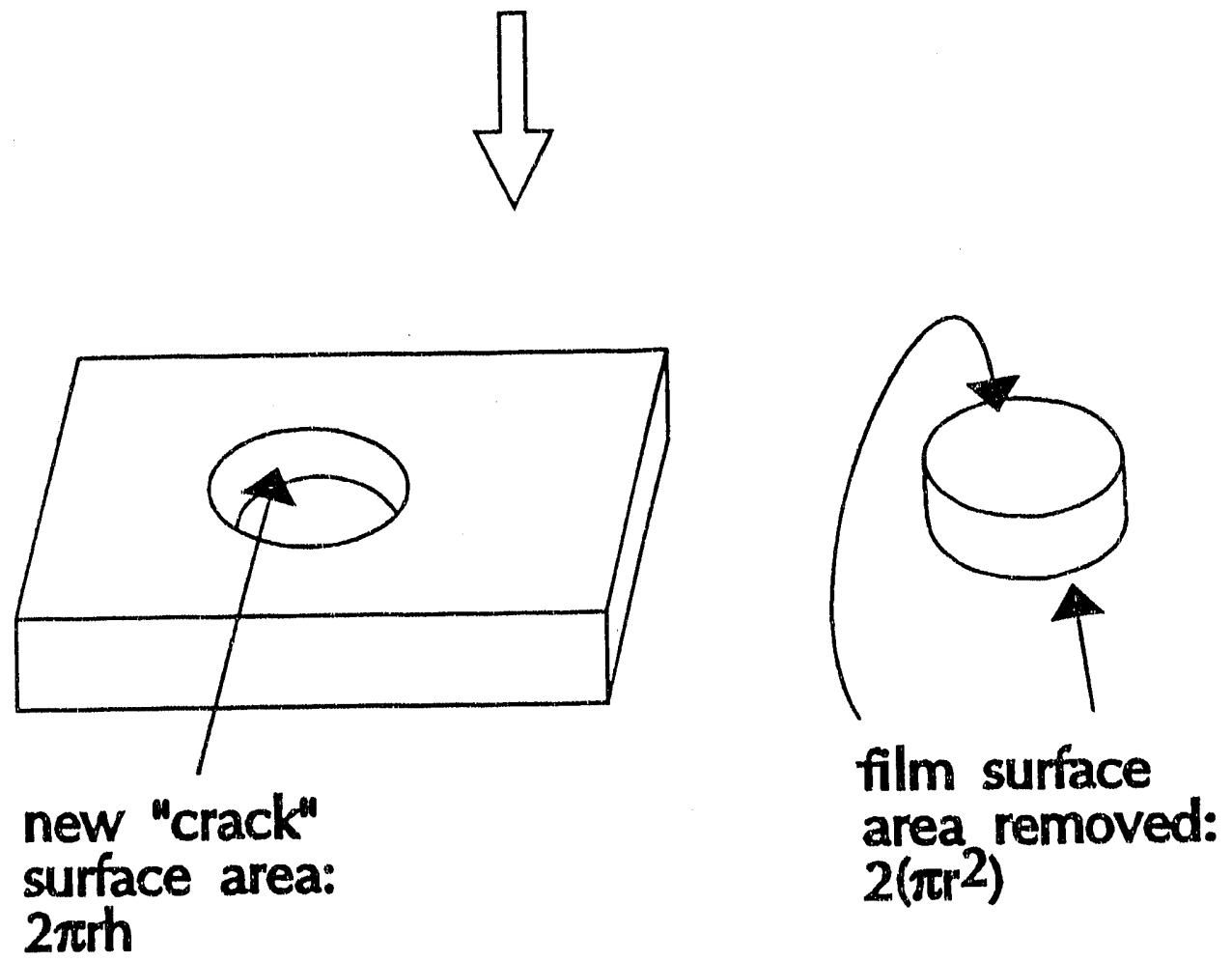

Figure 4.26 Competing surface areas are shown for the creation of a hole in the liquid over a pore. If the liquid is redistributed away from the pore rather than forming a film over it, the energy of the system will be reduced when the new "crack" surface area is less than the film surface area removed. 


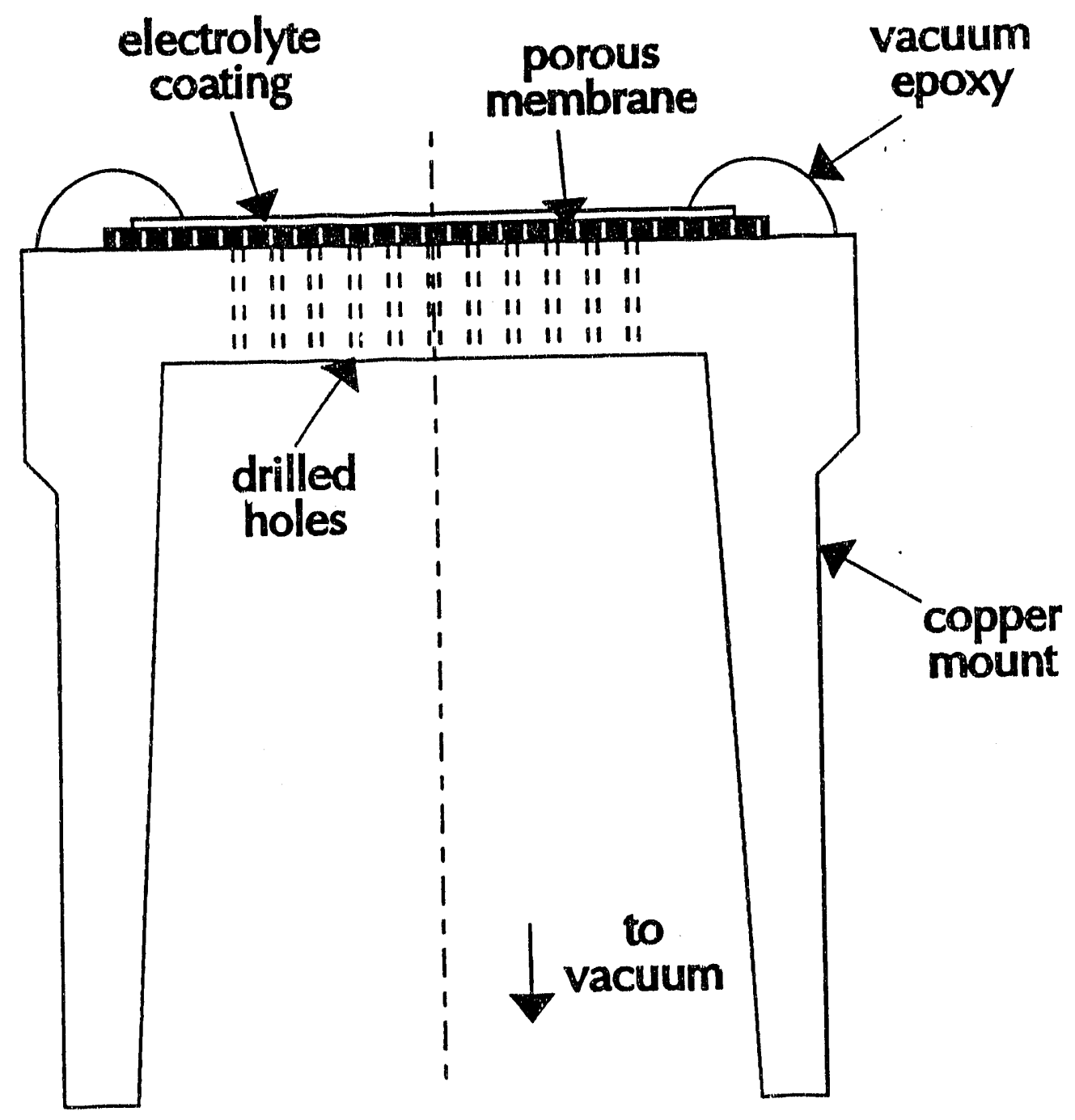

Figure 5.1 Apparatus used for leak testing of the electrolyte films. The coated membrane is sealed to a copper mount which is attached to a vacuum system with a mass spectrometer to detect helium. Helium is sprayed on the film from above. 


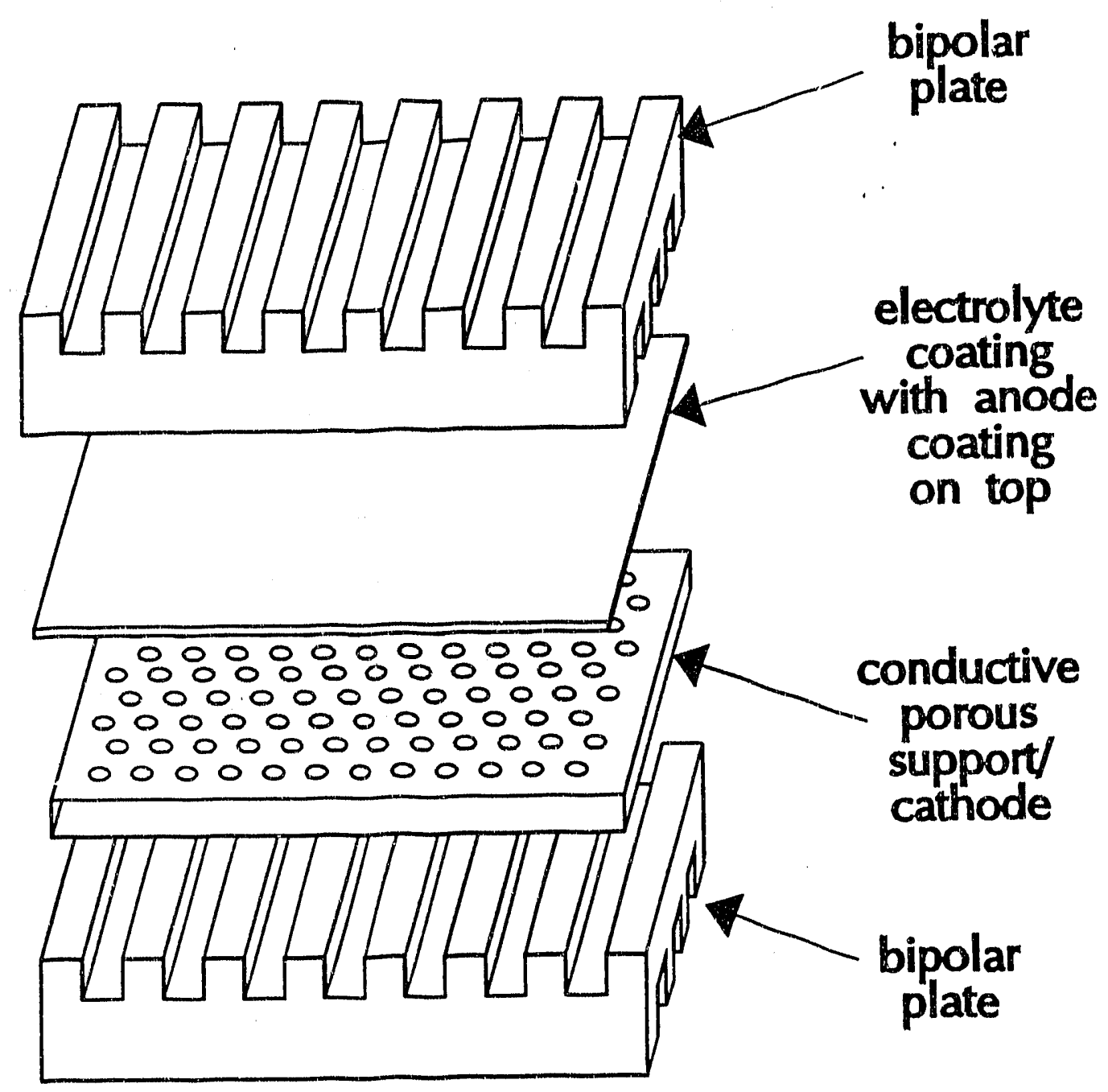

Figure 5.2 Diagram of a fuel cell stack utilizing bipolar plates. Voltage produced accross the electrolyte film must have a conductive path to the bipolar plates so that the cell voltages can be added. If the porous support is not electronically conductive, an external circuit is needed. 

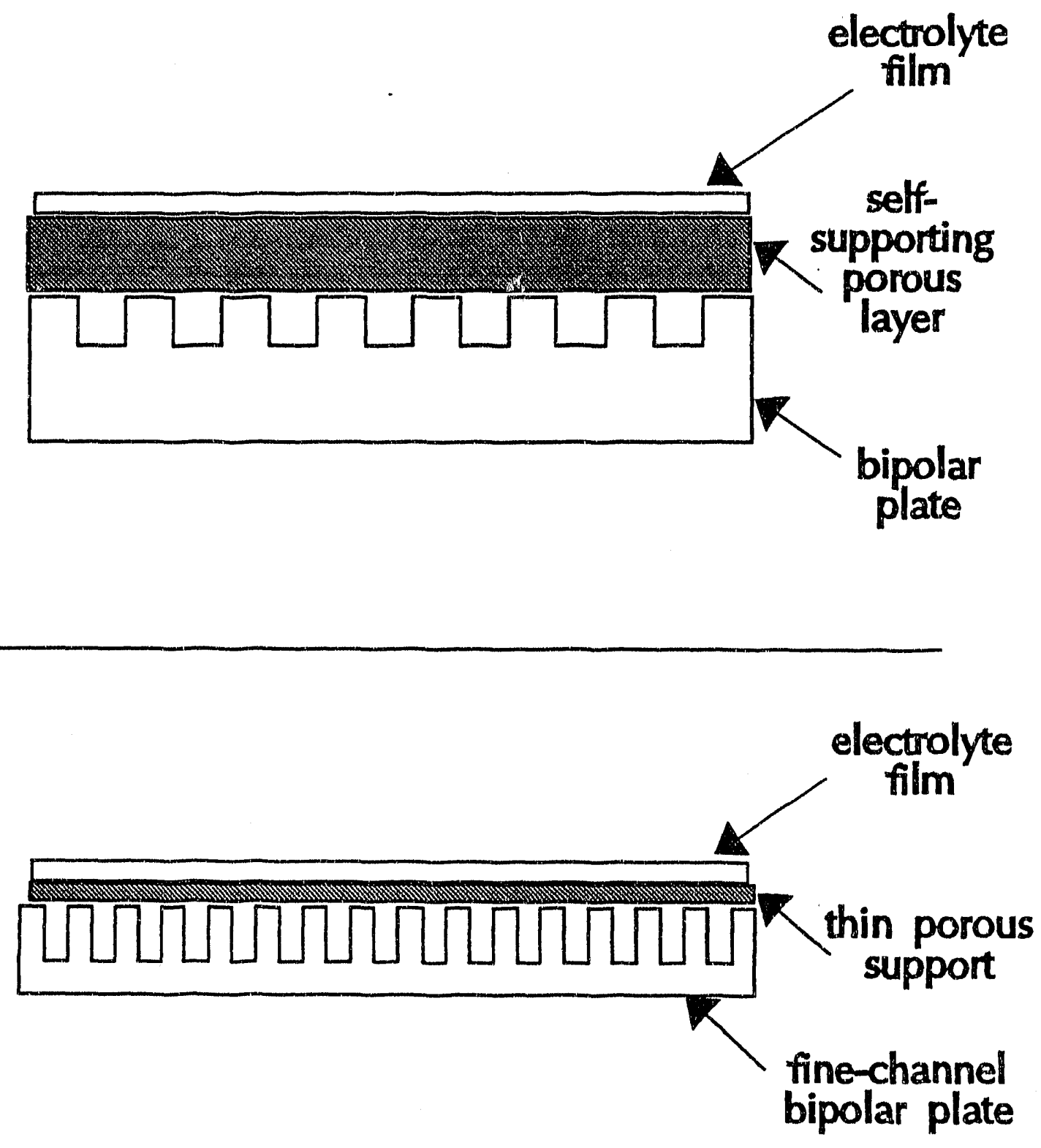

Figure 5.3 Diagrams of film/support/bipolar plate structures for two different channel spacings. If a plate with closely spaced deep channels is used, the film and porous support need not be self supporting; the bipolar plate could be used as a support during the fabrication of the porous layer and during the sol-gel coating step. 

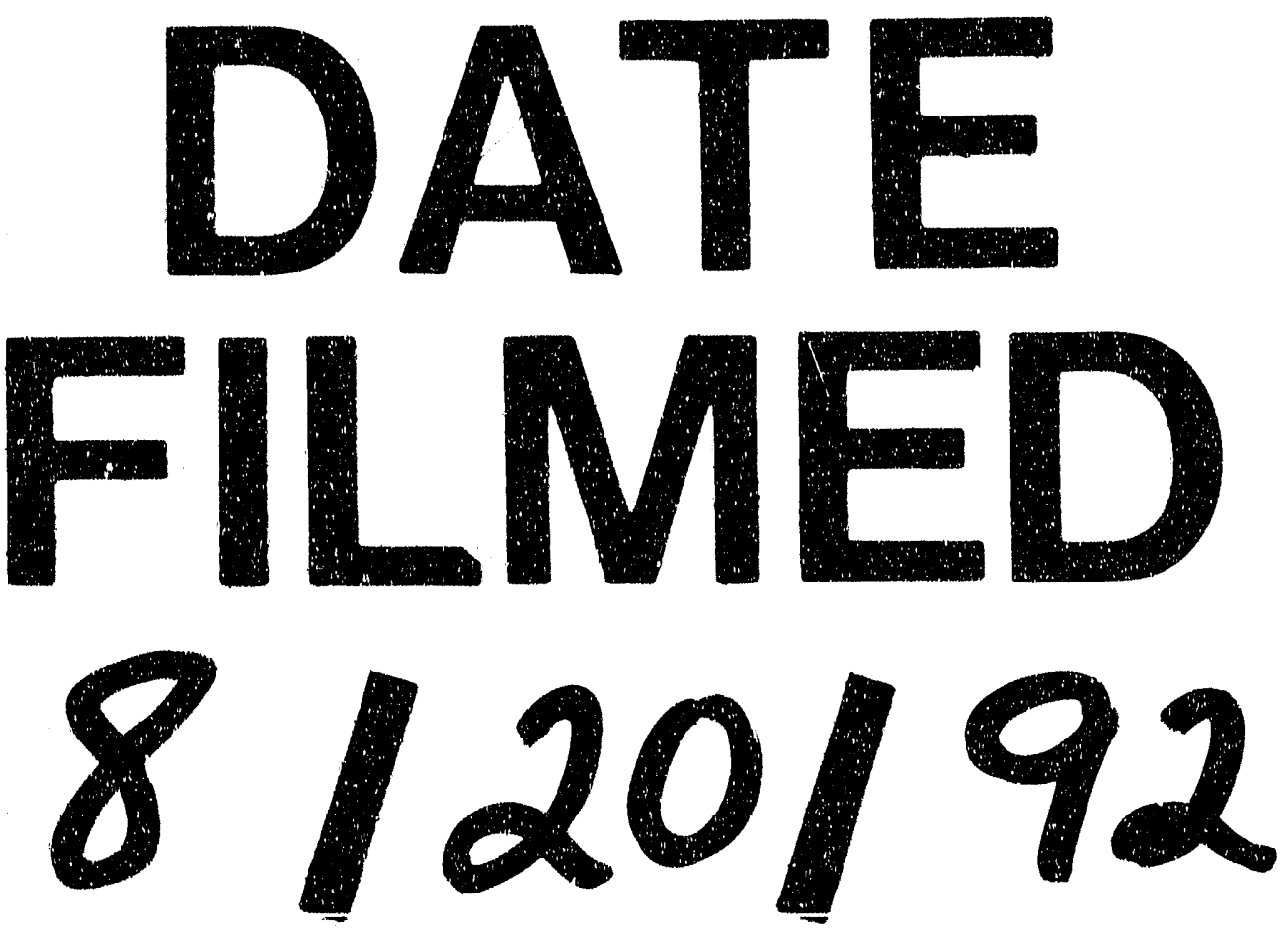
